TRANSACTIONS OF THE

AMERICAN MATHEMATICAL SOCIETY

Volume 359, Number 11, November 2007, Pages 5611-5651

S 0002-9947(07)04306-1

Article electronically published on June 22, 2007

\title{
LIMITS OF DISCRETE SERIES WITH INFINITESIMAL CHARACTER ZERO
}

\author{
HENRI CARAYOL AND A. W. KNAPP
}

\begin{abstract}
For a connected linear semisimple Lie group $G$, this paper considers those nonzero limits of discrete series representations having infinitesimal character 0 , calling them totally degenerate. Such representations exist if and only if $G$ has a compact Cartan subgroup, is quasisplit, and is acceptable in the sense of Harish-Chandra.

Totally degenerate limits of discrete series are natural objects of study in the theory of automorphic forms: in fact, those automorphic representations of adelic groups that have totally degenerate limits of discrete series as archimedean components correspond conjecturally to complex continuous representations of Galois groups of number fields. The automorphic representations in question have important arithmetic significance, but very little has been proved up to now toward establishing this part of the Langlands conjectures.

There is some hope of making progress in this area, and for that one needs to know in detail the representations of $G$ under consideration. The aim of this paper is to determine the classification parameters of all totally degenerate limits of discrete series in the Knapp-Zuckerman classification of irreducible tempered representations, i.e., to express these representations as induced representations with nondegenerate data.

The paper uses a general argument, based on the finite abelian reducibility group $R$ attached to a specific unitary principal series representation of $G$. First an easy result gives the aggregate of the classification parameters. Then a harder result uses the easy result to match the classification parameters with the representations of $G$ under consideration in representation-byrepresentation fashion. The paper includes tables of the classification parameters for all such groups $G$.
\end{abstract}

(0.1) Let $f=\sum_{n=1}^{\infty} a_{n} q^{n}$ be a classical modular newform, understood to be a normalized eigenform for the Hecke operators, of weight $k$ for $\Gamma_{0}(N)$. Then it is well known that the coefficients $a_{n}$ are algebraic integers and that once an embedding of the algebraic closure $\overline{\mathbb{Q}}$ of $\mathbb{Q}$ into $\overline{\mathbb{Q}}_{\ell}$ has been chosen, there exists a continuous representation $\tau$ of the Galois group $\operatorname{Gal}(\overline{\mathbb{Q}} / \mathbb{Q})$ into $G L\left(2, \overline{\mathbb{Q}}_{\ell}\right)$ such that the Frobenius element $F_{p}$ for any prime $p$ not dividing $N$ satisfies Trace $\tau\left(F_{p}\right)=a_{p}$.

In fact, two different situations arise corresponding to the two possibilities $k \geq 2$ and $k=1$. For weight $k \geq 2$, the construction of $\tau$ is straightforward, based on a decomposition of the cohomology of modular curves under Hecke operators. By contrast, for weight $k=1$, one uses congruences between forms of different weights to obtain, following Deligne and Serre, an "Artin" 2-dimensional representation, i.e.,

Received by the editors January 5, 2006.

2000 Mathematics Subject Classification. Primary 20G20, 22E45; Secondary 14L35.

Key words and phrases. Semisimple Lie group, discrete series, tempered representations.

(C)2007 American Mathematical Society Reverts to public domain 28 years from publication 
one with finite image. This difference between weight $\geq 2$ and weight 1 is mirrored by the archimedean component of the corresponding automorphic representation of $S L(2)$, which belongs to the discrete series in the first case and is a limit of discrete series in the second case.

(0.2) Let $G$ be a linear connected semisimple Lie group. Discrete series representations of $G$ are those having square integrable matrix coefficients; they are unitary representations. They exist if and only if the rank of $G$ coincides with the rank of a maximal compact subgroup $K$, and they were classified by Harish-Chandra [HC3]. In the classification each such representation is given nonuniquely by its HarishChandra parameter $\lambda$, which is a nonsingular linear functional on a compact Cartan subalgebra $\mathfrak{t}$ of the Lie algebra of $G$ and is integral when a certain specific linear functional $\rho$ is subtracted from it. Denote this representation by $\Theta(\lambda)$. Two such representations are equivalent if and only if their parameters are in the same orbit under the action of the Weyl group $W_{K}$ of $K$, which is generated by reflections with respect to the compact roots. Since $\lambda$ is nonsingular, $\lambda$ is dominant with respect to just one Weyl chamber $C$ of $i$ t. When the parameter $\lambda$ is moved by an integral amount so as to become possibly singular while remaining inside $C$, one can still associate a representation to the pair $(\lambda, C)$ (note that $C$ is no longer determined by $\lambda$ ), and the new representation $\Theta(\lambda, C)$ is called a limit of discrete series. This representation is irreducible or zero, and there is an easily stated criterion for deciding which is the case; specifically $\Theta(\lambda, C)$ is 0 if and only if $\lambda$ is orthogonal to some $C$-simple root that is compact. As above, $\Theta\left(\lambda^{\prime}, C^{\prime}\right)$ is equivalent to $\Theta(\lambda, C)$ if and only if $(\lambda, C)$ is conjugate to $\left(\lambda^{\prime}, C^{\prime}\right)$ by some element of $W_{K}$.

(0.3) One would like to be able to generalize the classical construction (0.1) to more general semisimple groups than $S L(2)$, as is predicted to be possible by the "Langlands philosophy." In the modern theory of automorphic forms, one starts from a semisimple algebraic group $G$ over $\mathbb{Q}$, and from an irreducible automorphic representation $\pi$ of the adelic group $G(\mathbb{A})$. It is well known that $\pi$ decomposes as an infinite tensor product of $p$-adic representations $\pi_{p}$ (one for each prime $p$ ) and one archimedean component $\pi_{\infty}$, which is a representation of $G(\mathbb{R})$. If the representation $\pi_{\infty}$ is algebraic in a sense that we do not want to make too precise here (roughly speaking this means that it is associated to parameters that are suitably integral), then an $\ell$-adic Galois representation should correspond to $\pi$. This should be the case in particular when $\pi_{\infty}$ is a discrete series representation or a limit of discrete series. For $G$ associated to a Hermitian symmetric domain and $\pi_{\infty}$ in the discrete series, a construction analogous to that explained in (0.1) above, but with Shimura varieties replacing modular curves, should in principle work, again according to the Langlands philosophy. Up to now this part of the Langlands program has been carried out in only a limited number of examples. It has been done, for instance, in [ Cl] for some inner forms of unitary groups over totally real fields. In these cases the construction produces representations of Galois groups, or more precisely "motives," that are regular, meaning that the weights from the point of view of algebraic geometry are all distinct. When the archimedean component is a limit of discrete series, there should still exist associated Galois representations, this time nonregular, i.e., having some coincidences between weights. There have been very few results in this direction; the construction should be indirect, using arithmetic congruences between automorphic representations. Such limits split into two categories: the nondegenerate ones, whose parameter $\lambda$ is orthogonal to 
no compact root (being singular, it is orthogonal to some root and the requirement is that this root cannot be a compact one); and the degenerate ones, for which $\lambda$ is orthogonal to some compact root (necessarily not a $C$-simple one). For the nondegenerate ones a construction analogous to that of Deligne-Serre should in principle still work and give the desired results, but it has actually been done in only a very limited number of cases. By contrast, degenerate limits never occur in the cohomology (neither étale nor coherent) of any Shimura variety, and consequently what happens with them is completely mysterious at present. What happens is closely related to the problem of understanding the arithmetic properties of certain Maass forms.

(0.4) We call a limit of discrete series totally degenerate if it is associated to the parameter $\lambda=0$ and to a Weyl chamber $C$ for which no $C$-simple root is compact. Such limits of discrete series have trivial infinitesimal character. Totally degenerate implies degenerate except in a group with no compact root, i.e., a group isogenous to a product of copies of $S L(2, \mathbb{R})$. Automorphic representations with totally degenerate limits of discrete series as archimedean components correspond conjecturally to Artin Galois representations; more accurately they should be associated with a morphism of the Galois group into the complex $L$-group ${ }^{L} G$. Thus such representations have a strong and simple arithmetic meaning. In addition, they correspond to each other by all known or conjectural cases of functoriality. So they play a very important role in the arithmetic theory of automorphic forms. However, very little is known about them except in the $S L(2)$ case.

(0.5) A possible approach to get a grip on automorphic representations with this kind of archimedean behavior has been proposed in $\mathrm{Ca}$ : it was shown in this article that automorphic representations of $S U(2,1)$ with limits of discrete series at archimedean places occur in the coherent cohomology of certain sheaves over some complex-analytic varieties generalizing Shimura varieties; these varieties are Griffiths-Schmid varieties, first studied by Griffiths as parameter spaces for variations of Hodge structures. Moreover $\mathrm{Ca}$ proved that there is a nontrivial cupproduct interaction between those forms and more classical ones that have discrete series (holomorphic or antiholomorphic) as infinite components. Similar results are expected for more general groups; at present a study of such properties has been undertaken only for quasisplit special unitary groups $S U(n, n)$ and $S U(n, n-1)$, but this kind of analysis should work more generally. In any case, to prove results similar to those of $\mathrm{Ca}$ requires very precise information about the totally degenerate limit of discrete series under consideration. More precisely, the computation of the cohomology itself can be done simply from the data $(0, C)$ defining the representation, by making use of a result of Soergel [So]. But to go further, especially to deal with cup products, one needs more, and for this purpose the full classification machinery of [KnZ] is important. For example, in Ca one uses the fact that the unique totally degenerate limit of discrete series for $S U(2,1)$ is in fact a principal series representation, one of the representations studied explicitly by Wallach Wa2 and more generally by K. Johnson and Wallach [oW]. This explicit information was used in $\mathrm{Ca}$ ] to obtain results relating to $(\mathfrak{g}, K)$ cohomology and automorphic forms, and to get a very precise and suitable tool to compute some related objects (cup products, for instance). The aim of the present paper is to get a correspondingly explicit description of totally degenerate limits of discrete series for more general groups. Let us explain what that means exactly. 
(0.6) Limits of discrete series are "tempered" representations. Langlands La proved that any irreducible tempered representation is a constituent of some "basic representation induced from discrete series," i.e., a constituent of a representation unitarily induced from a parabolic subgroup $M A N$ with a discrete series on $M$, a unitary character on $A$, and the trivial representation on $N$.

The paper $\mathrm{KnZ}$ classified irreducible tempered representations by making use of the more general notion of a basic representation, i.e., any representation unitarily induced from a parabolic subgroup $Q=M A N$ with a discrete series or limit of discrete series $\xi$ on $M$, a unitary character $e^{\nu}$ on $A$, and the trivial representation on $N$. Let us associate the set of data $(Q, \xi, \nu)$ to this representation. The paper $\mathrm{KnZ}$. proved as its Corollary 8.8 that every irreducible tempered representation is basic.

It turns out that nonzero basic representations can often be exhibited as basic by means of two quite different sets of data. A typical example of this phenomenon is precisely the totally degenerate limit of discrete series of $G=S U(2,1)$, which is realized at the same time as a spherical principal series. The full-fledged classification of irreducible tempered representations in $\mathrm{KnZ}$ makes use of a notion of nondegeneracy of the data for a basic representation. The data set $(Q, \xi, \nu)$ is degenerate if the representation is zero or if another realization is possible with a smaller parabolic subgroup; otherwise the data set is nondegenerate. Results in $\S 12$ of $\mathrm{KnZ}$ give criteria in terms of roots to decide whether $(Q, \xi, \nu)$ is degenerate. In particular a limit of discrete series $\Theta(\lambda, C)$ for $G$ is degenerate in the sense of (0.3) if and only if the data set $(G, \Theta(\lambda, C), 0)$ is degenerate in the present sense. It is immediate that every nonzero basic representation can be rewritten with nondegenerate data, and the classification theorem (Theorem 14.2 of [KnZ]) gives a criterion for a basic representation written with nondegenerate data to be irreducible, namely that a certain explicit finite abelian group $R$ is trivial. The theorem goes on to say that (a) the irreducible basic representations written with nondegenerate data exhaust the irreducible tempered representations and (b) two irreducible basic representations written with nondegenerate data are equivalent if and only if their sets of data are conjugate in the expected way.

(0.7) A nonzero limit of discrete series can therefore be written as an irreducible basic representation with nondegenerate data, and the data for this purpose are unique up to conjugacy. This set of data (or its conjugacy class) is what we take as the classification parameter of the limit of discrete series representation. For example, in the case of the totally degenerate limit of discrete series for $G=$ $S U(2,1)$, the nondegenerate data are those that appear when this representation is viewed as a spherical principal series. We can now ask a question that is natural from the point of view of the classification recalled above: given a degenerate limit of discrete series, find its classification parameter, that is to say, express it in terms of nondegenerate data. Not only is this a natural question, but also, as explained above, those parameters are often needed, in particular for some problems linked to automorphic forms.

(0.8) The purpose of the present article is to give a complete answer to this question in the case of totally degenerate limits of discrete series, i.e., those with trivial infinitesimal character. Even though the problem can be solved more easily and directly for some particular examples, e.g., for special unitary groups $S U(n, n)$ and $S U(n, n-1)$, we have found it necessary to have the solution in general. 
Indeed, as we have already suggested above, the importance of such representations lies in the fact that their automorphic counterparts are conjecturally linked to representations of the Galois group into the $L$-group. The fact that the family of all of them is stable under all possible functoriality is another reason to deal with all of them at once all in a general theorem.

The result we obtain is not available nor extractible from the existing literature, even if the techniques and machinery we are going to use in this article have been known to representation theorists for a long time; in particular, we make intensive use of an algorithm in $\mathrm{KnZ}$ associated to "generalized Schmid identities" that lies behind the classification of irreducible tempered representations. In the degenerate case this gives a tool for obtaining a realization of a basic representation as an induced representation with a new set of data corresponding to a smaller parabolic subgroup.

In an effort to make the paper more readable to nonexperts - especially people working in the theory of automorphic forms - we have given complete proofs of most of our results. We have also included some material that is certainly known to experts but is difficult or impossible to find in original sources or in texts.

The main result of the present article is Theorem 6.4 , which expresses any totally degenerate limit of discrete series $\Theta(0, C)$ as a representation induced from nondegenerate data, precisely described in a combinatorial way in terms of the Weyl chamber $C$. Tables in $\S 5$ and $\S 7$ and further discussion in $\S 7$ explain our main result in detail in particular cases.

(0.9) The proof of our theorem is a roundabout one, and what we do is approach it by stages, establishing coarser results first. Some important steps are taken in $\S 5$, where we prove that the sum of all the totally degenerate discrete series is equivalent to an induced representation $\operatorname{ind}_{Q_{\mathfrak{p}}}^{G}(\sigma \otimes 1 \otimes 1)$, specifically a unitary principal series attached to some minimal parabolic $Q_{\mathfrak{p}}=M_{\mathfrak{p}} A_{\mathfrak{p}} N_{\mathfrak{p}}$ with the trivial character on $A_{\mathfrak{p}}$ and a certain explicit 1-dimensional representation $\sigma$ of $M_{\mathfrak{p}}$ that is trivial on the identity component. This induced representation naturally decomposes as a certain sum of induced representations given with nondegenerate data; so we get in this way the set of such data corresponding to the set of all totally degenerate limits of discrete series. What remains to be done in $\S 6$ is to match factors on both sides. This is possible because we know quite a bit of detail about $\sigma$, which has been introduced in $\S 4$ : we have constructed it very precisely and then gathered a great deal of information about it. This patient construction is rewarded at the end because the theory of $\mathrm{KnZ}$ finally points to a canonical family of basic representations with nondegenerate data that exhibit the reducibility of $\operatorname{ind}_{Q_{\mathfrak{p}}}^{G}(\sigma \otimes 1 \otimes 1)$. With a careful argument that copes with all the nonuniqueness that arises, we can adjust the algorithm mentioned above so that it points to these same data, and we can then sort out the results of the algorithmic solution and obtain the representation-by-representation correspondence.

(0.10) The paper is organized as follows: The first two sections summarize some known material on Cayley transforms and their use in setting up the algorithm mentioned above. In $\S 3$ we work with two kinds of strongly orthogonal sequences of roots through which the necessary choices will enter our work. In $\S 4$ we use tools explicit or implicit in Satake [Sa] and Wallach Wa1] to construct the special 1-dimensional representation $\sigma$ of $M_{\mathfrak{p}}$ mentioned above that will determine the unitary principal series representation of interest. In Proposition 4.3 we derive an 
important technical property of $\sigma$ that relates it to the problem at hand. Theorems 5.1 and 5.2 show that this principal series representation contains each totally degenerate limit of discrete series representation with multiplicity one, and nothing else. The theory of the $R$ group is then applied routinely to yield the aggregate of classification parameters in Theorem 5.5. The main result of the paper is Theorem 6.4 , which gives the full answer to the problem and is proved by suitably rerouting what the algorithm says. Some examples of particular interest are given in $\S 7$. Tables in $\S 5$ and $\S 7$ show what Theorems 5.5 and 6.4 mean for all possible examples, up to conjugacy.

\section{Cayley transforms}

In this section we recall the behavior of Cayley transforms within the complexification of a real semisimple Lie algebra. For more detail, see $\S$ VI.7 of [Kn3]. Let $\mathfrak{g}=\mathfrak{k} \oplus \mathfrak{p}$ be a Cartan decomposition of a real semisimple Lie algebra, let $\theta$ be the corresponding Cartan involution, let $\mathfrak{g}^{\mathbb{C}}$ be the complexification of $\mathfrak{g}$, and let an overline indicate the conjugation of $\mathfrak{g}^{\mathbb{C}}$ with respect to $\mathfrak{g}$. Fix an invariant symmetric bilinear form $S$ on $\mathfrak{g}^{\mathbb{C}}$, and let $\langle\cdot, \cdot\rangle$ be the complex bilinear form induced on the dual of any Cartan subalgebra of $\mathfrak{g}^{\mathbb{C}}$. We assume that $S$ has been chosen so that the restriction of $\langle\cdot, \cdot\rangle$ to the real span of the roots is a real inner product. We put $|\alpha|^{2}=\langle\alpha, \alpha\rangle$.

Typical roots are denoted by $\alpha, \beta$, and $\xi$. Two members $\alpha$ and $\beta$ of the root system of $\mathfrak{g}^{\mathbb{C}}$ with respect to a Cartan subalgebra are said to be orthogonal, written $\alpha \perp \beta$, if $\langle\alpha, \beta\rangle=0$. They are strongly orthogonal, written $\alpha \perp \perp \beta$, if they are nonproportional and neither of $\alpha \pm \beta$ is a root; equivalently they are strongly orthogonal if they are orthogonal and it is false that both of $\alpha \pm \beta$ are roots. If the root system is irreducible, two orthogonal roots can fail to be strongly orthogonal only if the root system is of type $B_{n}, C_{n}$, or $F_{4}$ and the two roots are both short.

Fix a $\theta$ stable Cartan subalgebra $\mathfrak{h}$ of $\mathfrak{g}$. If $\mathfrak{b}$ and $\mathfrak{a}$ are the +1 and -1 eigenspaces of $\mathfrak{h}$ under $\theta$, then $\mathfrak{h}=\mathfrak{b} \oplus \mathfrak{a}$. Let $\Delta=\Delta\left(\mathfrak{g}^{\mathbb{C}}, \mathfrak{h}^{\mathbb{C}}\right)$ be the set of roots. Within the Weyl group of $\Delta$, the reflection in a root $\alpha$ is denoted by $s_{\alpha}$. Roots are imaginary-valued on $\mathfrak{b}$ and real-valued on $\mathfrak{a}$, and a root $\alpha$ is accordingly called

$$
\begin{aligned}
\text { imaginary } & \text { if } \alpha \text { is carried on } \mathfrak{b} \text {, i.e., is } 0 \text { on } \mathfrak{a}, \\
\text { real } & \text { if } \alpha \text { is carried on } \mathfrak{a} \text {, i.e., is } 0 \text { on } \mathfrak{b}, \\
\text { complex } & \text { if } \alpha \text { is nonzero on } \mathfrak{a} \text { and nonzero on } \mathfrak{b} .
\end{aligned}
$$

If $\mathfrak{g}_{\alpha}$ denotes the root space in $\mathfrak{g}^{\mathbb{C}}$ for the root $\alpha$, then

$$
\mathfrak{m}=\mathfrak{b} \oplus\left(\mathfrak{g} \cap \bigoplus_{\substack{\alpha \in \Delta, \alpha \text { imaginary }}} \mathfrak{g}_{\alpha}\right)
$$

is a $\theta$ stable reductive subalgebra contained in the centralizer of $\mathfrak{a}$ in $\mathfrak{g}$. It has the properties that $\mathfrak{m} \cap \mathfrak{a}=0$ and that the direct sum $\mathfrak{m} \oplus \mathfrak{a}$ is the centralizer $Z_{\mathfrak{g}}(\mathfrak{a})$ of $\mathfrak{a}$ in $\mathfrak{g}$, and it is given by the formula $\mathfrak{m}=\left\{X \in Z_{\mathfrak{g}}(\mathfrak{a}) \mid S(X, \mathfrak{a})=0\right\}$. The abelian subalgebra $\mathfrak{b}$ is a compact Cartan subalgebra of $\mathfrak{m}$, and thus $\operatorname{rank} \mathfrak{m}=\operatorname{rank}(\mathfrak{m} \cap \mathfrak{k})$.

If $\mathfrak{q}$ is any parabolic subalgebra of $\mathfrak{g}$, then $\mathfrak{l}=\mathfrak{q} \cap \theta \mathfrak{q}$ is the unique $\theta$ stable Levi factor of $\mathfrak{q}$. Define $\mathfrak{a}$ to be the intersection of $\mathfrak{p}$ with the center of $\mathfrak{q} \cap \theta \mathfrak{q}$, and let $\mathfrak{m}=\left\{X \in Z_{\mathfrak{l}}(\mathfrak{a}) \mid S(X, \mathfrak{a})=0\right\}$. The parabolic subalgebra $\mathfrak{q}$ is said to be cuspidal if rank $\mathfrak{m}=\operatorname{rank}(\mathfrak{m} \cap \mathfrak{k})$, hence if $\mathfrak{m}$ has a Cartan subalgebra $\mathfrak{b}$ contained in $\mathfrak{k}$. Then 
$\mathfrak{h}=\mathfrak{b} \oplus \mathfrak{a}$ is a $\theta$ stable Cartan subalgebra, and the $\mathfrak{m}$ just defined coincides with the $\mathfrak{m}$ constructed in (1.1). We say that $\mathfrak{q}$ and $\mathfrak{m} \oplus \mathfrak{a}$ are associated to $\mathfrak{h}=\mathfrak{b} \oplus \mathfrak{a}$.

For the $\mathfrak{m}$ associated to the $\theta$-stable Cartan subalgebra $\mathfrak{h}$, (1.1) allows us to identify the root system $\Delta\left(\mathfrak{m}^{\mathbb{C}}, \mathfrak{b}^{\mathbb{C}}\right)$ with the set of imaginary roots in $\Delta$ by restriction of members of $\Delta$ to $\mathfrak{b}^{\mathbb{C}}$. The root space for such an imaginary root $\alpha$ must lie completely within $(\mathfrak{m} \cap \mathfrak{k})^{\mathbb{C}}$ or completely within $(\mathfrak{m} \cap \mathfrak{p})^{\mathbb{C}}$, and we call $\alpha$ compact or noncompact accordingly. If we want to emphasize the role of $\mathfrak{m}$ in the construction, we may use the terms "m-compact" or "m-noncompact."

The Cayley transforms defined relative to a $\theta$ stable Cartan subalgebra $\mathfrak{h}=\mathfrak{b} \oplus \mathfrak{a}$ and its associated $\mathfrak{m} \oplus \mathfrak{a}$ are of two kinds, one $\mathbf{c}_{\beta}$ taken with respect to an imaginary noncompact root and the other $\mathbf{d}_{\alpha}$ taken with respect to a real root. Each is a certain kind of inner automorphism of $\mathfrak{g}^{\mathbb{C}}$.

The Cayley transform $\mathbf{c}_{\beta}$ with respect to an imaginary m-noncompact root $\beta$ takes us from the data $(\mathfrak{m}, \mathfrak{a}, \mathfrak{b})$ to data $\left(\mathfrak{m}_{*}, \mathfrak{a}_{*}, \mathfrak{b}_{*}\right)$ such that $\operatorname{dim} \mathfrak{a}_{*}=\operatorname{dim} \mathfrak{a}+1$ and $\operatorname{dim} \mathfrak{b}_{*}=\operatorname{dim} \mathfrak{b}-1$. If $E_{\beta}$ is a nonzero root vector for $\beta$, then $\overline{E_{\beta}}$ is a nonzero root vector for $-\beta$ and $S\left(E_{\beta}, \overline{E_{\beta}}\right)$ is $>0$. Normalizing, we may assume that

$$
S\left(E_{\beta}, \overline{E_{\beta}}\right)=2 /|\beta|^{2} .
$$

Then $\left[E_{\beta}, \overline{E_{\beta}}\right]=2|\beta|^{-2} H_{\beta}$, where $H_{\beta}$ is the member of $\mathfrak{h}$ such that $\left\langle H, H_{\beta}\right\rangle=\beta(H)$ for all $H \in \mathfrak{h}$. If we set $H_{\beta}^{\prime}=2|\beta|^{-2} H_{\beta}$, then $\left\{H_{\beta}^{\prime}, E_{\beta}, \overline{E_{\beta}}\right\}$ is a standard $\mathbb{C}$ basis for a copy of $\mathfrak{s l}(2, \mathbb{C})$ in $\mathfrak{g}^{\mathbb{C}}$, and $\left\{i H_{\beta}^{\prime}, E_{\beta}+\overline{E_{\beta}}, i\left(E_{\beta}-\overline{E_{\beta}}\right)\right\}$ is an $\mathbb{R}$ basis for a copy of $\mathfrak{s l}(2, \mathbb{R})$ within $\mathfrak{g}$. The automorphism $\mathbf{c}_{\beta}$ of $\mathfrak{g}^{\mathbb{C}}$ is defined by

$$
\mathbf{c}_{\beta}=\exp \left(\operatorname{ad} \frac{\pi}{4}\left(\overline{E_{\beta}}-E_{\beta}\right)\right) .
$$

It of course carries $\mathfrak{h}^{\mathbb{C}}$ to a new Cartan subalgebra of $\mathfrak{g}^{\mathbb{C}}$. Calculation gives

$$
\begin{aligned}
\mathbf{c}_{\beta}\left(H_{\beta}^{\prime}\right) & =E_{\beta}+\overline{E_{\beta}}, \\
\mathbf{c}_{\beta}\left(E_{\beta}-\overline{E_{\beta}}\right) & =E_{\beta}-\overline{E_{\beta}}, \\
\mathbf{c}_{\beta}\left(E_{\beta}+\overline{E_{\beta}}\right) & =-H_{\beta}^{\prime},
\end{aligned}
$$

and it follows that $\mathfrak{g} \cap \mathbf{c}_{\beta}\left(\mathfrak{h}^{\mathbb{C}}\right)=\operatorname{ker}\left(\left.\beta\right|_{\mathfrak{h}}\right) \oplus \mathbb{R}\left(E_{\beta}+\overline{E_{\beta}}\right)$. Thus the new Cartan subalgebra $\mathbf{c}_{\beta}\left(\mathfrak{h}^{\mathbb{C}}\right)$ is the complexification of

$$
\mathfrak{h}_{*}=\mathfrak{b}_{*} \oplus \mathfrak{a}_{*}=\left(\operatorname{ker}\left(\left.\beta\right|_{\mathfrak{b}}\right)\right) \oplus\left(\mathfrak{a} \oplus \mathbb{R}\left(E_{\beta}+\overline{E_{\beta}}\right)\right) .
$$

If $\mu$ is in the dual $\left(\mathfrak{h}^{\mathbb{C}}\right)^{\prime}$, then we can define $\mathbf{c}_{\beta}(\mu)$ in $\left(\mathfrak{h}_{*}^{\mathbb{C}}\right)^{\prime}$ by $\mathbf{c}_{\beta}(\mu)\left(H_{*}\right)=$ $\mu\left(\mathbf{c}_{\beta}^{-1}\left(H_{*}\right)\right)$. Under this definition, $\mathbf{c}_{\beta}$ carries roots to roots.

Let us consider the meaning of having two Cayley transforms commute. Suppose that $\beta$ and $\beta^{\prime}$ are $\mathfrak{m}$-noncompact roots. Then (1.3) defines $\mathbf{c}_{\beta}$ and $\mathbf{c}_{\beta^{\prime}}$, and hence the composition $\mathbf{c}_{\beta^{\prime}} \mathbf{c}_{\beta}$ is defined as an automorphism of $\mathfrak{g}^{\mathbb{C}}$. However, the interpretation of the composition as simply a product of expressions (1.3) does not take into account the effects on Cartan subalgebras: the factor $\mathbf{c}_{\beta}$ carries $\mathfrak{h}^{\mathbb{C}}$ to $\mathfrak{h}_{*}^{\mathbb{C}}$, and $\beta^{\prime}$ is not given as a root relative to $\mathfrak{h}_{*}^{\mathbb{C}}$. To interpret the composition as a succession of Cayley transforms, we should be working with $\mathbf{c}_{\mathbf{c}_{\beta}\left(\beta^{\prime}\right)} \mathbf{c}_{\beta}$ instead of $\mathbf{c}_{\beta^{\prime}} \mathbf{c}_{\beta}$. A condition is needed to be able to define $\mathbf{c}_{\mathbf{c}_{\beta}\left(\beta^{\prime}\right)}$, namely that $\mathbf{c}_{\beta}\left(\beta^{\prime}\right)$ is imaginary on $\mathfrak{h}_{*}$ and is $\mathfrak{m}_{*}$-noncompact.

The root $\mathbf{c}_{\beta}\left(\beta^{\prime}\right)$ will be imaginary if and only if $\beta$ and $\beta^{\prime}$ are orthogonal. However, orthogonality is not enough to make $\mathbf{c}_{\beta}\left(\beta^{\prime}\right)$ be $\mathfrak{m}_{*}$-noncompact; for this purpose 
we need to assume that $\beta$ and $\beta^{\prime}$ are strongly orthogonal. In fact, the strong orthogonality makes

$$
\left[E_{\beta}, E_{\beta^{\prime}}\right]=\left[E_{\beta}, E_{-\beta^{\prime}}\right]=\left[E_{-\beta}, E_{\beta^{\prime}}\right]=\left[E_{-\beta}, E_{-\beta^{\prime}}\right]=0 .
$$

Combining (1.3) and (1.4) shows that $\mathbf{c}_{\beta}$ fixes $E_{\beta^{\prime}}$ and $\overline{E_{\beta^{\prime}}}$. Hence $\mathbf{c}_{\beta}\left(\beta^{\prime}\right)$ is $\mathfrak{m}_{*}$-noncompact, and also

$$
\mathbf{c}_{\mathbf{c}_{\beta}\left(\beta^{\prime}\right)} \mathbf{c}_{\beta}=\mathbf{c}_{\beta^{\prime}} \mathbf{c}_{\beta} .
$$

A second application of (1.4) shows that $\mathbf{c}_{\beta^{\prime}} \mathbf{c}_{\beta}=\mathbf{c}_{\beta} \mathbf{c}_{\beta^{\prime}}$, so that $\mathbf{c}_{\mathbf{c}_{\beta}\left(\beta^{\prime}\right)} \mathbf{c}_{\beta}=$ $\mathbf{c}_{\mathbf{c}_{\beta^{\prime}}(\beta)} \mathbf{c}_{\beta^{\prime}}$ and the two Cayley transforms commute in every sense.

If $\beta$ and $\beta^{\prime}$ are merely orthogonal but not necessarily strongly orthogonal, the situation is more complicated. In particular, $\mathbf{c}_{\beta}\left(\beta^{\prime}\right)$ is $\mathfrak{m}_{*}$-compact; see Lemma 5.4 of $\mathrm{KnW}$ ] for details about what happens.

Partly because of the validity of (1.5) when the strongly orthogonal roots $\beta$ and $\beta^{\prime}$ are imaginary and $\mathfrak{m}$-noncompact, we shall use the same symbol for a root $\xi$ and its Cayley transform $\mathbf{c}_{\beta}(\xi)$ except possibly in the case that $\xi$ is $\beta$ itself. This convention will simplify the notation considerably when we work with a succession of several Cayley transforms.

The Cayley transform $\mathbf{d}_{\alpha}$ with respect to a real root $\alpha$ takes us from the data $(\mathfrak{m}, \mathfrak{a}, \mathfrak{b})$ to data $\left(\mathfrak{m}^{*}, \mathfrak{a}^{*}, \mathfrak{b}^{*}\right)$ such that $\operatorname{dim} \mathfrak{a}^{*}=\operatorname{dim} \mathfrak{a}-1$ and $\operatorname{dim} \mathfrak{b}^{*}=\operatorname{dim} \mathfrak{b}+1$. If $E_{\alpha}$ is a nonzero root vector for $\alpha$, then $\overline{E_{\alpha}}$ is a nonzero complex multiple of $E_{\alpha}$, and we can normalize $E_{\alpha}$ so that it is in $\mathfrak{g}$. Then $\theta E_{\alpha}$ is in $\mathfrak{g}$, is a root vector for $-\alpha$, and has the property that $S\left(E_{\alpha}, \theta E_{\alpha}\right)$ is $<0$. Normalizing, we may assume that $S\left(E_{\alpha}, \theta E_{\alpha}\right)=-2 /|\alpha|^{2}$. Then

$$
\left[E_{\alpha},-\theta E_{\alpha}\right]=2|\alpha|^{-2} H_{\alpha} .
$$

If we set $H_{\alpha}^{\prime}=2|\alpha|^{-2} H_{\alpha}$, then $\left\{H_{\alpha}^{\prime}, E_{\alpha},-\theta E_{\alpha}\right\}$ is a standard $\mathbb{C}$ basis for a copy of $\mathfrak{s t}(2, \mathbb{C})$ in $\mathfrak{g}^{\mathbb{C}}$, and in fact these basis elements all lie in $\mathfrak{g}$ and therefore form an $\mathbb{R}$ basis for a copy of $\mathfrak{s l}(2, \mathbb{R})$ within $\mathfrak{g}$. The automorphism $\mathbf{d}_{\alpha}$ of $\mathfrak{g}^{\mathbb{C}}$ is defined by

$$
\mathbf{d}_{\alpha}=\exp \left(\operatorname{ad} i \frac{\pi}{4}\left(\theta E_{\alpha}-E_{\alpha}\right)\right) \text {. }
$$

It of course carries $\mathfrak{h}^{\mathbb{C}}$ to a new Cartan subalgebra of $\mathfrak{g}^{\mathbb{C}}$. Calculation gives

$$
\begin{aligned}
\mathbf{d}_{\alpha}\left(H_{\alpha}^{\prime}\right) & =i\left(E_{\alpha}+\theta E_{\alpha}\right), \\
\mathbf{d}_{\alpha}\left(E_{\alpha}-\theta E_{\alpha}\right) & =E_{\alpha}-\theta E_{\alpha}, \\
\mathbf{d}_{\alpha}\left(E_{\alpha}+\theta E_{\alpha}\right) & =i H_{\alpha}^{\prime},
\end{aligned}
$$

and it follows that $\mathfrak{g} \cap \mathbf{d}_{\alpha}\left(\mathfrak{h}^{\mathbb{C}}\right)=\operatorname{ker}\left(\left.\alpha\right|_{\mathfrak{h}}\right) \oplus \mathbb{R}\left(E_{\alpha}+\theta E_{\alpha}\right)$. Thus the new Cartan subalgebra $\mathbf{d}_{\alpha}\left(\mathfrak{h}^{\mathbb{C}}\right)$ is the complexification of

$$
\mathfrak{h}^{*}=\mathfrak{b}^{*} \oplus \mathfrak{a}^{*}=\left(\mathfrak{b} \oplus i \mathbb{R}\left(E_{\alpha}+\theta E_{\alpha}\right)\right) \oplus \operatorname{ker}\left(\left.\alpha\right|_{\mathfrak{a}}\right) .
$$

In parallel with what happens for $\mathbf{c}_{\beta}$, formulas analogous to (1.4) and (1.5) hold for $\mathbf{d}_{\alpha}$ and $\mathbf{d}_{\alpha^{\prime}}$ when the real roots $\alpha$ and $\alpha^{\prime}$ are strongly orthogonal, and thus the Cayley transforms $\mathbf{d}_{\alpha}$ and $\mathbf{d}_{\alpha^{\prime}}$ commute under every interpretation of their definitions. For what happens when $\alpha$ and $\alpha^{\prime}$ are merely orthogonal, see Lemma 5.4 of $[\mathrm{KnW}]$. In analogy with the convention for the Cayley transform $\mathbf{c}_{\beta}$, we shall use the same symbol for a root $\xi$ and its Cayley transform $\mathbf{d}_{\alpha}(\xi)$ except possibly in the case that $\xi$ is $\alpha$ itself.

With $\alpha=\mathbf{c}_{\beta}(\beta)$ and with parameters defined suitably, $\mathbf{c}_{\beta}$ and $\mathbf{d}_{\alpha}$ are inverses of one another. In fact, we have only to define $E_{\alpha}$ as the member of $\mathfrak{g}$ given by 
$E_{\alpha}=\frac{1}{2}\left(-i H_{\beta}^{\prime}+i\left(E_{\beta}-\overline{E_{\beta}}\right)\right)$, and then we see readily from formulas (1.3), (1.6), and (1.7) that $\mathbf{d}_{\alpha} \mathbf{c}_{\beta}$ is the identity on $\mathfrak{g}^{\mathbb{C}}$.

\section{Generalized Schmid identities}

In this section we assemble certain facts from $\mathrm{KnZ}$ concerning the ingredients used in the classification of irreducible tempered representations of linear semisimple groups. Let $G$ be a connected linear semisimple Lie group with Lie algebra $\mathfrak{g}$, and let $\mathfrak{k}, \mathfrak{p}, \theta, \mathfrak{g}^{\mathbb{C}}, S,\langle\cdot, \cdot\rangle$, and $|\cdot|^{2}$ be as in $\S 1$. Since $G$ is linear, it has a complexification $G^{\mathbb{C}}$. The analytic subgroup of $G$ with Lie algebra $\mathfrak{k}$ is denoted by $K$. If $X$ and $Y$ are two subgroups of $G$, we define $W(X: Y)=N_{Y}(X) / Z_{Y}(X)$, the quotient of normalizer by centralizer; we use similar notation if one or both of $X$ and $Y$ are subalgebras of $\mathfrak{g}^{\mathbb{C}}$.

We begin with some remarks about limits of discrete series for $G$. According to [HC3, $G$ has discrete series representations if and only if $G$ and $K$ have equal rank. We write this condition as $\operatorname{rank} G=\operatorname{rank} K$ throughout the paper.

Limits of discrete series for $G$ itself were defined in full generality in [KnZ]. Discrete series have nonsingular infinitesimal character, and $\mathrm{KnZ}$ introduced limits of discrete series as certain representations with singular infinitesimal character. But in this paper it will be more convenient to allow discrete series to be special cases of limits of discrete series. The limits of discrete series were constructed from the discrete series, and thus they exist (in our present enlarged definition in which a singularity is no longer required) if and only if $\operatorname{rank} G=\operatorname{rank} K$.

Under the assumption that $\operatorname{rank} G=\operatorname{rank} K$, the construction of any limit of discrete series representation in $\mathrm{KnZ}$ begins with an application of the theory in $\mathrm{Zu}$ to a discrete series representation to move the infinitesimal character by an integral form while preserving dominance. Initially the construction produces not a representation of $G$, but a representation of the Lie algebra $\mathfrak{g}$ on a complex vector space of $K$-finite vectors, together with a compatible representation of $K$. This kind of pair of representations of $\mathfrak{g}$ and $K$ is known as a $(\mathfrak{g}, K)$ module. A roundabout argument in [KnZ, summarized as Theorem 1.1d, shows that this $(\mathfrak{g}, K)$ module is irreducible (or zero) and is infinitesimally unitary, i.e., carries an inner product with respect to which $\mathfrak{g}$ acts in skew-Hermitian fashion and $K$ acts in unitary fashion. Harish-Chandra's subquotient theorem in the form given in Le shows that any irreducible $(\mathfrak{g}, K)$ module is (nonuniquely) the underlying space of $K$-finite vectors for an irreducible representation of $G$ in a Hilbert space. Consequently we can apply Theorem 9 of [HC1] to see that the completion of the inner-product space for any irreducible infinitesimally unitary $(\mathfrak{g}, K)$ module becomes in a unique way the underlying space of a compatible irreducible unitary representation of $G$. Therefore the limit of discrete series can be regarded canonically as an irreducible unitary representation of $G$. This is the point of view of the present paper, and $(\mathfrak{g}, K)$ modules need play no further role.

A limit of discrete series is defined to be totally degenerate if it is nonzero and its infinitesimal character is 0 . The existence of totally degenerate limits of discrete series for $G$ implies that $G$ is acceptable in the sense of Harish-Chandra [HC4, i.e., that the linear functional $\rho$ in (0.2) (half the sum of the positive roots relative to a compact Cartan subalgebra) is integral. In fact, the effect of applying $[\mathrm{Zu}]$ as in the previous paragraph is to translate the infinitesimal character by an integral parameter. Since a discrete series with infinitesimal character $\lambda$ can exist only when 
$\lambda-\rho$ is integral, a limit of discrete series with infinitesimal character 0 can exist only when $0-\rho$ is integral, hence only when $\rho$ is integral. Thus two necessary conditions for the existence of a totally degenerate limit of discrete series are that $\operatorname{rank} G=\operatorname{rank} K$ and that $G$ is acceptable in the sense of Harish-Chandra. An example of a group with $\operatorname{rank} G=\operatorname{rank} K$ that is not acceptable is $S L(2, \mathbb{R}) /\{ \pm 1\}$. Shortly we shall see that a third necessary condition for the existence of a totally degenerate limit of discrete series is that $G$ is quasisplit. We shall see in Theorem 5.1 that conversely totally degenerate limits of discrete series always exist if $G$ has $\operatorname{rank} G=\operatorname{rank} K$, is quasisplit, and is acceptable in the sense of Harish-Chandra.

Returning to the general setting in which $G$ does not necessarily have $\operatorname{rank} G=$ $\operatorname{rank} K$, we need some information about the disconnectedness of Cartan subgroups and parabolic subgroups. A reference for this material is [Kn3], §§VII.7-8. Fix a $\theta$ stable Cartan subalgebra $\mathfrak{h}=\mathfrak{b} \oplus \mathfrak{a}$ of $\mathfrak{g}$, and let $B$ be the analytic subgroup of $K$ corresponding to $\mathfrak{b}$. For any real root $\alpha$, define

$$
\gamma_{\alpha}=\exp \left(2 \pi i|\alpha|^{-2} H_{\alpha}\right) ;
$$

this element centralizes $\mathfrak{h}$ and has order at most 2 . We let $F(B)$ be the subgroup of $B$ generated by all the elements $\gamma_{\alpha}$ for all real roots $\alpha$. Then $F(B) B$ is the centralizer of $\mathfrak{h}$ in $K$. Let $\mathfrak{q}=\mathfrak{m} \oplus \mathfrak{a} \oplus \mathfrak{n}$ be a cuspidal parabolic subalgebra associated to $\mathfrak{h}$. We write $Q=M A N$ for the Langlands decomposition of the corresponding parabolic subgroup, namely for the normalizer of $\mathfrak{q}$ in $G$. Then the center $Z_{M}$ of $M$ is given by

$$
Z_{M}=F(B) Z_{M_{0}},
$$

where $M_{0}$ is the identity component of $M$. The group $M_{0}$ is reductive and has $\operatorname{rank} M_{0}=\operatorname{rank}\left(M_{0} \cap K\right)$ and therefore has discrete series. We put $M^{\#}=M Z_{M}$, so that

$$
M^{\#}=M_{0} F(B) .
$$

Limits of discrete series representations for $M$ were defined and parametrized in [KnZ, and we follow [ KnZ in working with their global characters. Each such representation is built in stages. A limit of discrete series representation on the identity component $M_{0}$ is determined by its Harish-Chandra parameter $\lambda$, which gives the infinitesimal character, and by a Weyl chamber $C$ (or positive system for $\left.\Delta\left(\mathfrak{m}^{\mathbb{C}}, \mathfrak{b}^{\mathbb{C}}\right)\right)$ that makes $\lambda$ dominant; the representation exists if and only if $e^{\lambda-\rho}$ is well defined as a multiplicative character of $B, \rho$ being half the sum of the positive imaginary roots in any ordering. If $\lambda$ is nonsingular, then $C$ is unique and the representation is in the discrete series.

We write $\Theta^{M_{0}}(\lambda, C)$ for the global character. To extend the corresponding representation to $M^{\#}$, we adjoin a multiplicative character $\chi$ of $Z_{M}$ that is compatible with $\Theta^{M_{0}}(\lambda, C)$. Because of $(2.2)$, it is enough that $\chi$ be defined on $F(B)$; the compatibility condition is that $\chi$ agree with $e^{\lambda-\rho}$ on $F(B)$. The global character of the resulting limit of discrete series representation of $M^{\#}$ is denoted by $\Theta^{M^{\#}}(\lambda, C, \chi)$. Finally the limit of discrete series representations of $M$ are obtained by induction to $M$ of these representations of $M^{\#}$, and the global characters are written $\Theta^{M}(\lambda, C, \chi)$. An important property of these characters is as follows:

$$
\begin{aligned}
& \Theta^{M}(\lambda, C, \chi)=0 \text { if and only if }\langle\lambda, \alpha\rangle=0 \\
& \qquad \text { for some } C \text {-simple compact root } \alpha \in \Delta\left(\mathfrak{m}^{\mathbb{C}}, \mathfrak{b}^{\mathbb{C}}\right) .
\end{aligned}
$$


The sufficiency is known as the Hecht-Schmid identity and first appeared in HeS. The necessity is Theorem $1.1 \mathrm{~b}$ of $[\mathrm{KnZ}$. A necessary and sufficient condition for equality of the global characters $\Theta^{M}(\lambda, C, \chi)$ and $\Theta^{M}\left(\lambda^{\prime}, C^{\prime}, \chi^{\prime}\right)$ of two nonzero limits of discrete series is known and appears as Theorem 1.1c of [KnZ]: the condition is that $\chi=\chi^{\prime}$ and there exists a member of the normalizer of $B$ in $M$ carrying $(\lambda, C)$ to $\left(\lambda^{\prime}, C^{\prime}\right)$. Every nonzero limit of discrete series representation is irreducible, tempered, and unitary.

In the special case that the Cartan subalgebra is maximally noncompact, so that $\mathfrak{a}$ is maximal abelian in $\mathfrak{p}$, we use subscripts $\mathfrak{p}$ on various subalgebras and subgroups. The Cartan subalgebra is $\mathfrak{h}_{\mathfrak{p}}=\mathfrak{b}_{\mathfrak{p}} \oplus \mathfrak{a}_{\mathfrak{p}}$, and the cuspidal parabolic subalgebra is denoted by $Q_{\mathfrak{p}}=M_{\mathfrak{p}} A_{\mathfrak{p}} N_{\mathfrak{p}}$. A special feature of this case is that $M_{\mathfrak{p}}^{\#}=M_{\mathfrak{p}}$, so that

$$
M_{\mathfrak{p}}=\left(M_{\mathfrak{p}}\right)_{0} F\left(B_{\mathfrak{p}}\right) .
$$

For the case in which $\operatorname{rank} G=\operatorname{rank} K$, we mentioned just before (2.1) that $G$ can have totally degenerate limits of discrete series only if $G$ is quasisplit. To see this necessity, let $\pi$ be a totally degenerate limit of discrete series. Using HarishChandra's subquotient theorem in [HC2, we embed $\pi$ as a subquotient of some nonunitary principal series representation, i.e., one induced from an irreducible finite-dimensional representation of a minimal parabolic subgroup $Q_{\mathfrak{p}}=M_{\mathfrak{p}} A_{\mathfrak{p}} N_{\mathfrak{p}}$ of $G$. The latter representation must then have matching infinitesimal character 0 , and hence the inducing representation of $M_{\mathfrak{p}}$ must have infinitesimal character 0. Since the infinitesimal character of a finite-dimensional representation is nonsingular, $M_{\mathfrak{p}}$ must have no roots. It follows that $M_{\mathfrak{p}}$ is abelian, hence that $G$ is quasisplit.

Returning to the general case with $\mathfrak{h}=\mathfrak{b} \oplus \mathfrak{a}$ and $Q=M A N$, suppose that $\Theta^{M}(\lambda, C, \chi)$ is a limit of discrete series character of $M$. If $\nu$ is a real-valued linear functional on $\mathfrak{a}$, we let

$$
\Theta^{M A}(\lambda, C, \chi, \nu)=\Theta^{M}(\lambda, C, \chi) \otimes e^{i \nu},
$$

which is a global character for $M A$. If we tensor with the trivial character of $N$ and induce to $G$, we obtain global characters

$$
\operatorname{ind}_{Q}^{G} \Theta^{M A}(\lambda, C, \chi, \nu),
$$

which are called basic characters. In the special case that $\Theta^{M}(\lambda, C, \chi)$ is a discrete series character of $M$, we call $\operatorname{ind}_{Q}^{G} \Theta^{M A}(\lambda, C, \chi, \nu)$ a basic character induced from discrete series. It is known that basic characters are unaffected by changing the subgroup $N$ of the parabolic subgroup $Q$. A basic representation is a representation whose global character is a basic character.

A basic representation does not necessarily uniquely determine the data of a basic character, even up to conjugacy. Sometimes a basic character can be written in terms of data from two different Cartan subalgebras obtained from one another by one or more Cayley transforms. This kind of change is typically made in stages, and the change at a single stage is implemented by means of a "generalized Schmid identity." Generalized Schmid identities are of two possible kinds, the distinction having to do with the relationship between the centers of the two groups $M$. The notation is as follows. We start from a $\theta$ stable Cartan subalgebra $\mathfrak{h}^{*}=\mathfrak{b}^{*} \oplus \mathfrak{a}^{*}$ and an associated parabolic subgroup $Q^{*}=M^{*} A^{*} N^{*}$. Let $\widetilde{\alpha}$ be a noncompact imaginary root, i.e., a noncompact root of $\left(\mathfrak{m}^{* \mathbb{C}}, \mathfrak{b}^{* \mathbb{C}}\right)$, and suppose that the Cayley transform $\mathbf{c}_{\widetilde{\alpha}}$ leads from the data $\left(\mathfrak{m}^{*}, \mathfrak{a}^{*}, \mathfrak{b}^{*}\right)$ to data $(\mathfrak{m}, \mathfrak{a}, \mathfrak{b})$ and that $\alpha=\mathbf{c}_{\widetilde{\alpha}}(\widetilde{\alpha})$. 
According to Lemma 2.2 of $\mathrm{KnZ}$, the inclusion mapping of $Z_{M}$ into $Z_{M}\left(M \cap M^{* \#}\right)$ yields an isomorphism

$$
Z_{M} /\left\{1, \gamma_{\alpha}\right\} Z_{M_{0}} Z_{M^{*}} \cong Z_{M}\left(M \cap M^{* \#}\right) /\left(M \cap M^{* \#}\right),
$$

the groups on either side of (2.6) have order at most 2 , and the groups have order exactly 2 if and only if the reflection $s_{\widetilde{\alpha}}$ is in $W\left(B^{*}: M^{*}\right)$, i.e., if and only if the root reflection $s_{\widetilde{\alpha}}$ has a representative in $M^{*}$.

To state the identities, let us introduce

$$
C=\text { a Weyl chamber in } i \mathfrak{b}^{*},
$$

$C^{s^{\widetilde{\alpha}}}=$ unique Weyl chamber of $i \mathfrak{b}$ containing the orthogonal projection

$$
\operatorname{Proj}_{i \mathfrak{b}}(C)=\operatorname{Proj}_{i \mathfrak{b}}\left(s_{\widetilde{\alpha}} C\right) \text {, }
$$

$\rho_{\alpha}=$ half sum of roots of $\left(\mathfrak{g}^{\mathbb{C}},(\mathfrak{a} \oplus \mathfrak{b})^{\mathbb{C}}\right)$ whose restriction to $\mathfrak{a}$ is $c \alpha$ with $c>0$,

$\zeta=$ multiplicative character of $\left\{1, \gamma_{\alpha}\right\}$ given by $\zeta\left(\gamma_{\alpha}\right)=(-1)^{2\left\langle\rho_{\alpha}, \alpha\right\rangle /|\alpha|^{2}}$.

According to Theorem 4.3 of $\mathrm{KnZ}$, if $\widetilde{\alpha}$ is $C$-simple as a noncompact root of $\left(\mathfrak{m}^{* \mathbb{C}}, \mathfrak{b}^{* \mathbb{C}}\right)$ and if data $\lambda, \chi$, and $\nu$ for $\Theta^{M^{*} A^{*}}(\lambda, C, \chi, \nu)$ are such that

(i) $\lambda-\rho_{M^{*}}$ is $\mathfrak{b}^{*}$-integral, and $e^{\lambda-\rho_{M^{*}}}$ agrees with $\chi$ on $Z_{M^{*}} \cap B^{*}$,

(ii) $\langle\lambda, \widetilde{\alpha}\rangle=0$,

(iii) $\lambda$ is $C$-dominant (and hence also $s_{\widetilde{\alpha}} C$-dominant),

then either (a) or (b) holds as follows:

(a) If $s_{\widetilde{\alpha}}$ is not in $W\left(B^{*}: M^{*}\right)$, then $Z_{M}=\left\{1, \gamma_{\alpha}\right\} Z_{M_{0}} Z_{M^{*}}$ and

$\Theta^{M^{*} A^{*}}(\lambda, C, \chi, \nu)+\Theta^{M^{*} A^{*}}\left(\lambda, s_{\widetilde{\alpha}} C, \chi, \nu\right)=\operatorname{ind}_{P \cap M^{*} A^{*}}^{M^{*} \Theta^{*}} \Theta^{M A}\left(\left.\lambda\right|_{\mathfrak{b}}, C^{s_{\widetilde{\alpha}}}, \zeta \otimes \chi, \nu \oplus 0\right)$, where $C^{s \widetilde{\alpha}}$ and $\zeta$ are defined as in (2.7).

(b) If $s_{\widetilde{\alpha}}$ is in $W\left(B^{*}: M^{*}\right)$, then $\left|Z_{M} /\left\{1, \gamma_{\alpha}\right\} Z_{M_{0}} Z_{M^{*}}\right|=2$. Let $C^{s_{\widetilde{\alpha}}}$ and $\zeta$ be defined as in (2.7), and let $(\zeta \oplus \chi)^{+}$and $(\zeta \oplus \chi)^{-}$denote the two extensions of $\zeta \otimes e^{\left.\left(\lambda-\rho_{M}\right)\right|_{\mathfrak{b}}} \otimes \chi$ to $Z_{M}$. Then

$$
\begin{aligned}
\Theta^{M^{*} A^{*}}(\lambda, C, \chi, \nu) & =\operatorname{ind}_{P \cap M^{*} A^{*}}^{M^{*} A^{*}} \Theta^{M A}\left(\left.\lambda\right|_{\mathfrak{b}}, C^{s_{\widetilde{\alpha}}},(\zeta \otimes \chi)^{+}, \nu \oplus 0\right) \\
& =\operatorname{ind}_{P \cap M^{*} A^{*}}^{M^{*} A^{*}} \Theta^{M A}\left(\left.\lambda\right|_{\mathfrak{b}}, C^{s_{\widetilde{\alpha}}},(\zeta \otimes \chi)^{-}, \nu \oplus 0\right) .
\end{aligned}
$$

The displayed formulas in (a) and (b) above will be called generalized Schmid identities of types (a) and (b), respectively. In both cases we can of course extend the global characters to $M^{*} A^{*} N^{*}$ by adjoining the trivial character of $N^{*}$, and then we can induce to $G$, obtaining a character identity for basic characters for $G$. In the case of (b), or in the case of (a) if the second term on the left side is 0 , these identities allow us to take a basic character given in terms of data for $\left(\mathfrak{m}^{*}, \mathfrak{a}^{*}, \mathfrak{b}^{*}\right)$ and rewrite it in terms of data for $(\mathfrak{m}, \mathfrak{a}, \mathfrak{b})$. In the case of $(a)$, we can use the analog of (2.4) for $\left(\mathfrak{m}^{*}, \mathfrak{a}^{*}, \mathfrak{b}^{*}\right)$ to determine whether the second term on the left side is 0 . The order of $W\left(B^{*}: M^{*}\right)$ determines whether (a) or (b) is the applicable generalized Schmid identity.

In the terminology of $\S 12$ of $\mathrm{KnZ}$, we say that a basic character written as $\operatorname{ind}_{Q^{*}}^{G} \Theta^{M^{*} A^{*}}(\lambda, C, \chi, \nu)$ is given by nondegenerate data if, for each root $\widetilde{\alpha}$ of $\left(\mathfrak{m}^{* \mathbb{C}}, \mathfrak{b}^{* \mathbb{C}}\right)$ with $\langle\lambda, \widetilde{\alpha}\rangle=0$, the reflection $s_{\widetilde{\alpha}}$ is not in $W\left(B^{*}: M^{*}\right)$. The character is automatically nonzero in this case. Proposition 12.1 of $[\mathrm{KnZ}$ says that the data set of a nonzero basic character $\operatorname{ind}_{Q^{*}}^{G} \Theta^{M^{*} A^{*}}(\lambda, C, \chi, \nu)$ is nondegenerate if 
and only if $\Theta^{M^{*}} A^{*}(\lambda, C, \chi, \nu)$ is not the full left side of a generalized Schmid identity of type (a) or type (b). It follows that every nonzero basic character can be rewritten in terms of nondegenerate data.

A nonzero basic character is not necessarily irreducible. When written in terms of nondegenerate data, it is irreducible if and only if a certain computable finite abelian group $R$ is trivial. We discuss this group $R$ further when we need it in $\S 5$.

By a theorem of Langlands La (see also Harish-Chandra HC4 and Trombi $[\mathrm{Tr})$, every irreducible tempered representation of $G$ is a subrepresentation of a basic representation induced from discrete series and hence is unitary.

The classification theorems say that every irreducible tempered representation is basic and therefore can be written with nondegenerate data. Two irreducible tempered characters written in terms of nondegenerate data are equal if and only if their sets of data are conjugate in a suitable sense. The conjugacy class of this set of data constitutes the set of classification parameters of the representation.

The goal of this paper is to identify the classification parameters of each totally degenerate limit of discrete series. In principle this problem can be solved by taking the given representation and iterating generalized Schmid identities until we arrive at nondegenerate data, but it is not clear a priori how to abstract the results from this algorithm so as to obtain a useful theorem. It turns out that a different approach yields a tidier formulation of the result. What we shall do in effect is to overshoot the answer and pass all the way to a maximally noncompact Cartan subgroup; then we shall retrace some of our steps. For this purpose we need to be able to invert generalized Schmid identities.

Thus let us start from a $\theta$ stable Cartan subalgebra $\mathfrak{h}=\mathfrak{b} \oplus \mathfrak{a}$ and an associated parabolic subgroup $Q=M A N$. Let $\alpha$ be a real root, and suppose that the Cayley transform $\mathbf{d}_{\alpha}$ leads from the data $(\mathfrak{m}, \mathfrak{a}, \mathfrak{b})$ to data $\left(\mathfrak{m}^{*}, \mathfrak{a}^{*}, \mathfrak{b}^{*}\right)$ and that $\widetilde{\alpha}=\mathbf{d}_{\alpha}(\alpha)$. According to Theorem 6.1 of [KnZ], a given character written as

$$
\operatorname{ind}_{P \cap M^{*} A^{*}}^{M^{*} A^{*}} \Theta^{M A}\left(\lambda_{M}, C_{M}, \chi_{M}, \nu_{M}\right)
$$

is the right side of a generalized Schmid identity of type (a) or (b) obtained from $\widetilde{\alpha}$ as above if and only if $\left\langle\nu_{M}, \alpha\right\rangle=0$ and

$$
\chi_{M}\left(\gamma_{\alpha}\right)=(-1)^{2\left\langle\rho_{\alpha}, \alpha\right\rangle /|\alpha|^{2}},
$$

where $\rho_{\alpha}$ is given by $(2.7)$. When these conditions are satisfied, the definitions of $\lambda, \chi$, and $\nu$ are

$$
\begin{gathered}
\lambda= \begin{cases}\lambda_{M} & \text { on } \mathfrak{b}, \\
0 & \text { on } H_{\widetilde{\alpha}},\end{cases} \\
\chi=\left.\chi_{M}\right|_{Z_{M^{*}}} \quad \text { and } \quad \nu=\left.\nu_{M}\right|_{\mathfrak{a}^{*}},
\end{gathered}
$$

and the Weyl chamber $C$ in $i \mathfrak{b}^{*}$ can be taken to be any chamber such that

(i) $\lambda_{M} \oplus 0$ is $C$-dominant,

(ii) $(\bar{C} \cap i \mathfrak{b}) \cap C_{M}$ has nonempty interior, and

(iii) $\widetilde{\alpha}$ is $C$-positive.

It will be important for us that the definitions of $\lambda, \chi$, and $\nu$ do not depend on $C_{M}$. Thus if we have several basic characters differing only in what chambers are involved, then we can use each or none of them as the right side of a generalized Schmid identity, and all the left sides will involve the common values of $\lambda, \chi$, and $\nu$. 


\section{Strongly ORThOGONAL SYSTEMS OF ROOTS}

In this section we construct the sequences of noncompact roots that will incorporate the required element of choice into our determination of classification parameters, and we study the properties of these sequences. We continue with the notation of $\S 2$. We assume further that $\mathfrak{g}$ has a compact Cartan subalgebra, and we take $\mathfrak{t}$ to be such a subalgebra that is contained in $\mathfrak{k}$. Fix a maximally noncompact $\theta$ stable Cartan subalgebra $\mathfrak{h}_{\mathfrak{p}}$. Our objective in this section is to examine maximal sets of strongly orthogonal roots.

We begin with strongly orthogonal sequences of real roots relative to $\mathfrak{h}_{\mathfrak{p}}$, where the situation is easy and is handled by the following proposition. We shall be doing successive Cayley transforms $\mathbf{d}_{\alpha}$ with respect to the members of such a sequence, using an inverted generalized Schmid identity for each member of the sequence. Part of the effect of the assumed strong orthogonality is to ensure that these Cayley transforms commute.

Proposition 3.1. Under the assumptions that rank $\mathfrak{g}=$ rank $\mathfrak{k}$ and that $\mathfrak{h}_{\mathfrak{p}}=$ $\mathfrak{b}_{\mathfrak{p}} \oplus \mathfrak{a}_{\mathfrak{p}}$ is maximally noncompact, there exists a strongly orthogonal sequence of real roots on $\mathfrak{h}_{\mathfrak{p}}$ whose restrictions to $\mathfrak{a}_{\mathfrak{p}}$ span the dual $\mathfrak{a}_{\mathfrak{p}}^{\prime}$.

Proof. Since rank $\mathfrak{g}=$ rank $\mathfrak{k}$, Problem 10a of Chapter VI of [Kn3] (along with its answer in the Hints for Solutions of Problems) shows that there exists $k \in K$ with $\operatorname{Ad}(k)=\theta$ on $\mathfrak{g}$ and in particular on $\mathfrak{h}_{\mathfrak{p}}$. That is, $\operatorname{Ad}(k)$ is +1 on $\mathfrak{b}_{\mathfrak{p}}$ and -1 on $\mathfrak{a}_{\mathfrak{p}}$. This element $k$ yields an element $w$ of the Weyl group $W\left(\mathfrak{h}_{\mathfrak{p}}^{\mathbb{C}}: \mathfrak{g}^{\mathbb{C}}\right)$ that acts this way. Applying Proposition 2.72 (Chevalley's Lemma) of Kn3 recursively, we obtain an orthogonal sequence of roots $\alpha_{1}, \ldots, \alpha_{l}$ that vanish on $\mathfrak{b}_{\mathfrak{p}}$ and have $w=s_{\alpha_{1}} \cdots s_{\alpha_{l}}$. Then $\alpha_{1}, \ldots, \alpha_{l}$ are orthogonal real roots and span $\mathfrak{a}_{\mathfrak{p}}^{\prime}$. We shall adjust these roots to make them strongly orthogonal.

Without loss of generality, suppose that $\mathfrak{g}$ is simple. The roots of $\mathfrak{g}$ have at most two lengths, and there can be no problem unless two of them, say $\alpha_{i}$ and $\alpha_{j}$, are short and are such that $\alpha_{i} \pm \alpha_{j}$ are roots. In this case, we replace $\alpha_{i}$ and $\alpha_{j}$ in the sequence by $\alpha_{i}+\alpha_{j}$ and $\alpha_{i}-\alpha_{j}$. The orthogonality of the sequence is maintained, and the two new roots, being long, cannot interfere with strong orthogonality. By successive elimination in this way of pairs that are orthogonal but not strongly orthogonal, we keep reducing the number of such pairs, and ultimately we arrive at the desired sequence.

In the context of Proposition 3.1, we shall need to expand roots on $\mathfrak{h}_{\mathfrak{p}}$ in terms of the strongly orthogonal sequence of real roots and a remainder term carried on $\mathfrak{b}_{\mathfrak{p}}$. We need this expansion only when $\mathfrak{g}$ is quasisplit. Confining our attention to $\mathfrak{g}$ quasisplit makes the expansion easier to state for complex roots, and thus we limit the statement of the following proposition to $\mathfrak{g}$ quasisplit.

Proposition 3.2. Let $\mathfrak{g}$ be quasisplit and simple with rank $\mathfrak{g}=$ rank $\mathfrak{k}$, and suppose that $\mathfrak{g}$ is not of type $G_{2}$. Let $\mathfrak{h}_{\mathfrak{p}}=\mathfrak{b}_{\mathfrak{p}} \oplus \mathfrak{a}_{\mathfrak{p}}$ be a $\theta$ stable maximally noncompact Cartan subalgebra of $\mathfrak{g}$, and let $\alpha_{1}, \ldots, \alpha_{l}$ be a strongly orthogonal set of real roots in $\Delta\left(\mathfrak{g}^{\mathbb{C}}, \mathfrak{h}_{\mathfrak{p}}^{\mathbb{C}}\right)$ that spans $\mathfrak{a}_{\mathfrak{p}}^{\prime}$. Lexicographic use of $\alpha_{1}, \ldots, \alpha_{l}$ determines uniquely a set $\Delta^{+}=\Delta^{+}\left(\mathfrak{g}^{\mathbb{C}}, \mathfrak{h}_{\mathfrak{p}}^{\mathbb{C}}\right)$ of positive roots. Suppose that $\beta$ is a positive root other than some $\alpha_{i}$. Then the expansion of $\beta$ in terms of $\alpha_{1}, \ldots, \alpha_{l}$ is of one of six kinds: If 
$\beta$ is real, then $\beta$ is given by one of the four kinds

$$
\begin{aligned}
& \beta=\frac{1}{2} \alpha_{i} \pm \frac{1}{2} \alpha_{r} \pm \frac{1}{2} \alpha_{s} \pm \frac{1}{2} \alpha_{t} \quad \text { with }\left\{\begin{array}{l}
|\beta|=\left|\alpha_{i}\right|=\left|\alpha_{r}\right|=\left|\alpha_{s}\right|=\left|\alpha_{t}\right|, \\
i<\min \{r, s, t\} \\
\text { and the indices } r, s, t \text { distinct }
\end{array}\right. \\
& \beta=\frac{1}{2} \alpha_{i} \pm \alpha_{r} \pm \frac{1}{2} \alpha_{s} \quad \text { with }\left\{\begin{array}{l}
|\beta|=\left|\alpha_{i}\right|=\left|\alpha_{s}\right|>\left|\alpha_{r}\right|, \\
i<\min \{r, s\}, \text { and } r \neq s,
\end{array}\right. \\
& \beta=\alpha_{i} \pm \frac{1}{2} \alpha_{r} \pm \frac{1}{2} \alpha_{s} \quad \text { with }\left\{\begin{array}{l}
|\beta|=\left|\alpha_{r}\right|=\left|\alpha_{s}\right|>\left|\alpha_{i}\right|, \\
i<\min \{r, s\}, \text { and } r \neq s,
\end{array}\right. \\
& \beta=\frac{1}{2} \alpha_{i} \pm \frac{1}{2} \alpha_{r} \quad \text { with }\left\{\begin{array}{l}
|\beta|<\left|\alpha_{r}\right|=\left|\alpha_{i}\right|, \\
i<r .
\end{array}\right.
\end{aligned}
$$

If $\beta$ is complex, then all roots have the same length and $\beta$ is of one of the two kinds

$$
\begin{array}{ll}
\beta=\frac{1}{2} \alpha_{i}+\omega & \text { with } \quad\left\{\begin{array}{l}
|\beta|=\left|\alpha_{i}\right|, \\
\omega \text { nonzero, carried on } \mathfrak{b}_{\mathfrak{p}},
\end{array}\right. \\
\beta=\frac{1}{2} \alpha_{i} \pm \frac{1}{2} \alpha_{r}+\omega \quad \text { with } \quad\left\{\begin{array}{l}
|\beta|=\left|\alpha_{i}\right|=\left|\alpha_{r}\right|, i<r, \\
\omega \text { nonzero, carried on } \mathfrak{b}_{\mathfrak{p}} .
\end{array}\right.
\end{array}
$$

Remark. Strongly orthogonal sets $\alpha_{1}, \ldots, \alpha_{l}$ of the type in the statement of the proposition exist by virtue of Proposition 3.1.

Proof. As to the uniqueness of $\Delta^{+}$, adjoin some vectors to the end of the sequence $\alpha_{1}, \ldots, \alpha_{l}$ so that the result is an orthogonal basis of $\mathfrak{a}_{\mathfrak{p}}^{\prime} \oplus i \mathfrak{b}_{\mathfrak{p}}^{\prime}$. In the corresponding ordering, the sign of every root is determined by $\alpha_{1}, \ldots, \alpha_{l}$ since $\mathfrak{g}$ quasisplit implies that no root is carried on $\mathfrak{b}_{\mathfrak{p}}$. Thus the system $\Delta^{+}$of positive roots is independent of what elements were adjoined to form the orthogonal basis.

Let us classify the kinds of roots that are possible. One case is that $\beta$ is real. Since $\alpha_{1}, \ldots, \alpha_{l}$ spans $\mathfrak{a}_{\mathfrak{p}}^{\prime}$, we have $\beta=\sum_{j} c_{j} \alpha_{j}$. Taking the inner product with $\alpha_{j}$ gives $c_{j}=\left\langle\beta, \alpha_{j}\right\rangle /\left|\alpha_{j}\right|^{2}$, so that

$$
\beta=\sum_{j=1}^{l} \frac{\left\langle\beta, \alpha_{j}\right\rangle}{\left|\alpha_{j}\right|^{2}} \alpha_{j} .
$$

We double this equation, take the norm squared, and divide by $|\beta|^{2}$ to obtain

$$
4=\sum_{j=1}^{l} \frac{4\left\langle\beta, \alpha_{j}\right\rangle^{2}}{\left|\alpha_{j}\right|^{2}|\beta|^{2}}
$$

Let us discard those terms on the right side of (3.8) that are 0. Since $\beta$ is not some $\pm \alpha_{j}$ and since we have discarded $G_{2}$ by hypothesis, each remaining term is 1 or 2 . So there are at most 4 nonzero terms in (3.7). The first one has positive coefficient since $\beta$ is assumed positive; let us write $\alpha_{i}$ for that $\alpha_{j}$. The indices for the remaining terms will be $r, s, t$ as necessary. 
First suppose that $|\beta|=\left|\alpha_{i}\right|$. Apart from permuting indices after $i,(3.8)$ reads $4=1+1+1+1$ or $4=1+2+1$. These yield (3.1), (3.2), and

$$
\beta=\frac{1}{2} \alpha_{i} \pm \frac{1}{2} \alpha_{r} \pm \frac{1}{2} \alpha_{s} \quad \text { with }\left\{\begin{array}{l}
|\beta|=\left|\alpha_{i}\right|=\left|\alpha_{s}\right|<\left|\alpha_{r}\right|, \\
i<\min \{r, s\}, \text { and } r \neq s .
\end{array}\right.
$$

If (3.9) holds, then $2\left\langle\beta, \pm \alpha_{r}\right\rangle /|\beta|^{2}=2$, so that $\pm \alpha_{r}-2 \beta$ is a root. Since

$$
\pm \alpha_{r}-2 \beta= \pm \alpha_{r}-\left(\alpha_{i} \pm \alpha_{r} \pm \alpha_{s}\right)=-\alpha_{i} \mp \alpha_{s},
$$

we obtain a contradiction to the assumption that $\alpha_{i}$ and $\alpha_{s}$ are strongly orthogonal. Thus (3.9) cannot occur.

Next suppose that $|\beta|>\left|\alpha_{i}\right|$. Then (3.8) reads $4=2+1+1$ or $4=2+2$. These yield (3.3) and

$$
\beta=\alpha_{i} \pm \alpha_{r} \quad \text { with } \quad\left\{\begin{array}{l}
|\beta|>\left|\alpha_{i}\right|=\left|\alpha_{r}\right|, \\
i<r,
\end{array}\right.
$$

which cannot occur because $\alpha_{i}$ and $\alpha_{r}$ are strongly orthogonal.

Finally suppose that $|\beta|<\left|\alpha_{i}\right|$. Then (3.8) reads $4=2+2$ or $4=2+1+1$. These yield (3.4) and

$$
\beta=\frac{1}{2} \alpha_{i} \pm \frac{1}{2} \alpha_{r} \pm \frac{1}{2} \alpha_{s} \quad \text { with }\left\{\begin{array}{l}
|\beta|=\left|\alpha_{r}\right|=\left|\alpha_{s}\right|<\left|\alpha_{i}\right|, \\
i<\min \{r, s\}, \text { and } r \neq s .
\end{array}\right.
$$

Except for various signs, the situation of (3.10) can be transformed into (3.9) by interchanging $i$ and $r$, and we saw that (3.9) cannot occur because of the strong orthogonality of $\alpha_{1}, \ldots, \alpha_{l}$. Thus (3.10) cannot occur either, and the only possibilities are (3.1) through (3.4) if $\beta$ is real.

The other case is that $\beta$ is complex. Since $\mathfrak{b}_{\mathfrak{p}} \neq 0, \mathfrak{g}$ is not split. Since $\mathfrak{g}$ is assumed quasisplit, $\mathfrak{g}^{\mathbb{C}}$ must admit a nontrivial outer automorphism. Therefore all roots have the same length. Let us write $\beta=\varepsilon+\omega$ for the decomposition of $\beta$ according to $\mathfrak{h}_{\mathfrak{p}}=\mathfrak{a}_{\mathfrak{p}} \oplus \mathfrak{b}_{\mathfrak{p}}$. Then $\bar{\beta}=\varepsilon-\omega=-\theta \beta$ is a root of the same length that is not a multiple of $\beta$. Consequently

$$
\frac{2\langle\beta, \bar{\beta}\rangle}{|\beta|^{2}}
$$

equals $-1,0$, or +1 . We can rule out +1 since $\beta-\bar{\beta}$ would have to be an imaginary root and we know that there are no imaginary roots. In the two remaining cases, we have $|\varepsilon|^{2}=c_{0}|\beta|^{2}$ with $c_{0}=1 / 4$ if (3.11) is -1 and $c_{0}=1 / 2$ if (3.11) is 0 .

We write $\varepsilon=\sum_{j=1}^{l} c_{j} \alpha_{j}$ and compute that $c_{j}=\left\langle\beta, \alpha_{j}\right\rangle /\left|\alpha_{j}\right|^{2}$. Then $\varepsilon=$ $\sum_{j=1}^{l}\left(\left\langle\beta, \alpha_{j}\right\rangle /\left|\alpha_{j}\right|^{2}\right) \alpha_{j}$, and it follows that

$$
4 c_{0}=\sum_{j=1}^{l} \frac{4\left\langle\beta, \alpha_{j}\right\rangle^{2}}{\left|\alpha_{j}\right|^{2}|\beta|^{2}}=\#\left\{\alpha_{j} \mid\left\langle\beta, \alpha_{j}\right\rangle \neq 0\right\},
$$

the second equality following since all roots have the same length. If $c_{0}=1 / 4$, then the right side of (3.12) is 1 , and $\beta$ has to be as in (3.5). If $c_{0}=1 / 2$, then the right side of (3.12) is 2 , and $\beta$ has to be as in (3.6).

Now we examine strongly orthogonal sequences of roots relative to the compact Cartan subalgebra $t$. Since each such root is imaginary, each such root is compact or noncompact. Our interest will be in the case that all the roots are noncompact 
and some other conditions are satisfied. The reason for insisting on strong orthogonality is analogous to the reason for the strong orthogonality in the case above for real roots: We shall be doing successive Cayley transforms $\mathbf{c}_{\beta}$ with respect to the members of such a sequence, using a (direct) generalized Schmid identity for each member of the sequence. The strong orthogonality ensures, among other things, that these Cayley transforms commute.

The noncompactness and the other conditions we impose make the discussion more complicated than it was in Proposition 3.1 for real roots. A preliminary step is to normalize root vectors suitably. For each pair $\pm \beta$ of noncompact roots, we normalize $E_{\beta}$ as in (1.2) and define $E_{-\beta}=\overline{E_{\beta}}$. Then

$$
E_{\beta}+E_{-\beta} \text { and } i\left(E_{\beta}-E_{-\beta}\right) \text { are in } \mathfrak{g} \text {. }
$$

To get started, let $\Delta^{+}\left(\mathfrak{g}^{\mathbb{C}}, \mathfrak{t}^{\mathbb{C}}\right)$ be a positive system for $\Delta\left(\mathfrak{g}^{\mathbb{C}}, \mathfrak{t}^{\mathbb{C}}\right)$. We make the following definition, which is slightly different from the one in $\S 4$ of [KnW]. A sequence $\alpha_{1}, \ldots, \alpha_{l}$ of noncompact positive roots in $\Delta$ will be called a fundamental sequence for $\Delta^{+}\left(\mathfrak{g}^{\mathbb{C}}, \mathfrak{t}^{\mathbb{C}}\right)$ if

(i) $\alpha_{1}, \ldots, \alpha_{l}$ are strongly orthogonal,

(ii) $\alpha_{j}$ is a simple root in the subsystem of roots orthogonal to $\alpha_{1}, \ldots, \alpha_{j-1}$, for $1 \leq j \leq l$,

(iii) $\mathfrak{a}_{\mathfrak{p}}=\sum_{j=1}^{l} \mathbb{R}\left(E_{\alpha_{j}}+E_{-\alpha_{j}}\right)$ is maximal abelian in $\mathfrak{p}$.

This definition is internally consistent in the following senses: the subsystem of roots orthogonal to $\alpha_{1}, \ldots, \alpha_{j-1}$ is certainly a root system, (3.13) shows that each $E_{\alpha_{j}}+E_{-\alpha_{j}}$ is in $\mathfrak{g}$, and the noncompactness of $\alpha_{j}$ implies that $E_{\alpha_{j}}$ and $E_{-\alpha_{j}}$ lie in $\mathfrak{p}^{\mathbb{C}}$.

To be absolutely complete, we should insert the following lemma, which was not included in $\mathrm{KnW}$ ] but was implicitly applied in $\S 4$ of $[\mathrm{KnW}$ in the construction of certain sequences of noncompact positive roots; these sequences will appear in the proof of Proposition 3.4 below.

Lemma 3.3. If $\Omega$ is a root system and $\alpha$ is in $\Omega$, then $\Omega^{\prime}=\{\beta \in \Omega \mid \beta \perp \perp \alpha\}$ is empty or is a root subsystem of $\Omega$ with the property that when $\beta_{1}$ and $\beta_{2}$ are in $\Omega^{\prime}$ and $\beta_{1}+\beta_{2}$ is in $\Omega$, then $\beta_{1}+\beta_{2}$ is in $\Omega^{\prime}$.

Proof. Without loss of generality we may assume that $\Omega$ is irreducible, that there are roots of two lengths, that the root $\alpha$ is short, that there exists a long root $\gamma$, and that $|\gamma|^{2}=2|\alpha|^{2}$. Let $\beta_{1}$ and $\beta_{2}$ be in $\Omega^{\prime}$, and suppose $\beta_{1}+\beta_{2}$ is in $\Omega$. We are to prove that $\beta_{1}+\beta_{2} \perp \perp \alpha$.

We are given $\beta_{1} \perp \perp \alpha$ and $\beta_{2} \perp \perp \alpha$, and hence $\beta_{1}+\beta_{2} \perp \alpha$. If $\beta_{1}+\beta_{2} \perp \perp \alpha$ fails, then $\beta_{1}+\beta_{2}+\alpha$ is in $\Omega$. Hence $\beta_{1}+\beta_{2}$ is short, and at least one of $\beta_{1}$ and $\beta_{2}$ is short. Say $\beta_{2}$ is short. Then we have

$$
\frac{2\left\langle\beta_{1}+\beta_{2}+\alpha, \beta_{1}\right\rangle}{\left|\beta_{1}\right|^{2}}=2+\frac{2\left\langle\beta_{2}, \beta_{1}\right\rangle}{\left|\beta_{1}\right|^{2}}+0 .
$$

This is $>0$ since $\beta_{2}$ is short. Therefore $\left(\beta_{1}+\beta_{2}+\alpha\right)-\beta_{1}=\beta_{2}+\alpha$ is a root, in contradiction to the hypothesis $\beta_{2} \perp \perp \alpha$.

Proposition 3.4. Under the assumption that $\mathfrak{g}$ has a compact Cartan subalgebra $\mathfrak{t} \subseteq \mathfrak{k}$, any positive system $\Delta^{+}\left(\mathfrak{g}^{\mathbb{C}}, \mathfrak{t}^{\mathbb{C}}\right)$ of the root system $\Delta\left(\mathfrak{g}^{\mathbb{C}}, \mathfrak{t}^{\mathbb{C}}\right)$ has a fundamental sequence. 
Proof. We may assume that $\Delta=\Delta\left(\mathfrak{g}^{\mathbb{C}}, \mathfrak{t}^{\mathbb{C}}\right)$ is irreducible. First let us dispose of $G_{2}$. Take $\alpha_{1}$ to be a simple root that is noncompact; one exists as long as $\mathfrak{g}$ is noncompact. Then take $\alpha_{2}$ to be the unique positive root orthogonal to $\alpha_{1}$. This is noncompact in every case, and the sequence $\alpha_{1}, \alpha_{2}$ has the required properties.

When $\Delta$ is not of type $G_{2}$, Proposition 4.5 of $\mathrm{KnW}$ shows that there exists a sequence $\alpha_{1}, \ldots, \alpha_{l}$ of positive noncompact roots such that

(a) the $\alpha_{j}$ form a strongly orthogonal set,

(b) $\mathfrak{a}_{p}=\sum_{j=1}^{l} \mathbb{R}\left(E_{\alpha_{j}}+E_{-\alpha_{j}}\right)$ is maximal abelian in $\mathfrak{p}$,

(c) $\alpha_{j}$ is a simple root in the subsystem of roots strongly orthogonal to all of $\alpha_{1}, \ldots, \alpha_{j-1}$, which is empty or is a root system by virtue of Lemma 3.3 above,

(d) for each positive noncompact root $\beta$, the first $\alpha_{j}$ such that $\alpha_{j}$ is not strongly orthogonal to $\beta$ satisfies $\left|\alpha_{j}\right| \geq|\beta|$.

Then (a) and (b) prove (i) and (iii), and (c) proves (ii) if all roots have the same length.

It is possible to give a general argument for (ii) in the case that there are two root lengths, but for brevity we give a case-by-case argument that uses the detailed analysis in $\S 4$ of $\mathrm{KnW}$. If $\Delta$ is of type $C_{n}$ or $F_{4}$ with some long root noncompact, then the sequence constructed in $\mathrm{KnW}$ ] has all roots long, and the system of roots orthogonal to $\alpha_{1}, \ldots, \alpha_{j-1}$ coincides with the system strongly orthogonal to those roots. Hence the sequence constructed in $\mathrm{KnW}$ satisfies (ii) in these cases.

If $\mathfrak{g}$ is noncompact with $\Delta$ of type $F_{4}$ and with all noncompact roots short, then the sequence consists of one noncompact simple root from $\Delta$, and (ii) holds.

For $\Delta$ of type $B_{n}, \mathrm{KnW}$ shows that the constructed sequence can be taken to be of the form

$$
\text { certain } e_{i}-e_{j}, \quad \text { possible } e_{k}, \quad \text { corresponding } e_{i}+e_{j} \text {. }
$$

Orthogonality relative to a long root is the same as strong orthogonality, and hence there is no problem with (ii) for the first members of (3.14). One easily checks that the possible $e_{k}$ and the corresponding $e_{i}+e_{j}$ are all simple in the system of roots orthogonal to all the members of the first group, and hence each of them is simple in the smaller system of roots orthogonal to all members of the sequence that precede it. This handles (ii) for $\Delta$ of type $B_{n}$.

The last case is that $\mathfrak{g}$ is noncompact with $\Delta$ of type $C_{n}$ and with all noncompact roots short. We follow the construction of [KnW] step by step. Selection of the first noncompact simple root $\alpha_{1}=e_{i}-e_{j}$ means that the strongly orthogonal system consists of all roots not involving indices $i$ and $j$, while the larger orthogonal system consists of $\pm\left(e_{i}+e_{j}\right)$ in addition to all roots not involving indices $i$ and $j$. The root $e_{i}+e_{j}$ is simple in the latter system. The next step produces some $\alpha_{2}=e_{s}-e_{t}$. Indices $s$ and $t$ are to be discarded, except that $\pm\left(e_{s}+e_{t}\right)$ remains in the system of roots orthogonal to $\alpha_{1}, \alpha_{2}$. Again $e_{s}+e_{t}$ is simple in the latter system. The construction continues in this way, and each selection of an $\alpha_{j}$ is seen to be of a simple root in the system of roots orthogonal to $\alpha_{1}, \ldots, \alpha_{j-1}$. This completes the proof.

\section{The Representation $\sigma$ of $M_{\mathfrak{p}}$}

In this section, under some assumptions on $G$, we explicitly construct a certain 1-dimensional representation $\sigma$ of the group $M_{\mathfrak{p}}$ of a minimal parabolic subgroup 
of $G$ and prove a key technical result about it. It will be seen in $\S 5$ that the unitary principal series representation built from $\sigma$ and the trivial character of $A_{\mathfrak{p}}$ is equivalent with the direct sum of all totally degenerate limits of discrete series representations, each occurring with multiplicity 1 .

We continue with notation as in $\S 2$. Fix a maximally noncompact $\theta$ stable Cartan subalgebra $\mathfrak{h}_{\mathfrak{p}}=\mathfrak{b}_{\mathfrak{p}} \oplus \mathfrak{a}_{\mathfrak{p}}$ of $\mathfrak{g}$, and let $M_{\mathfrak{p}}$ be the centralizer of $\mathfrak{a}_{\mathfrak{p}}$ in $K$. Following standard terminology, we refer to the nonzero simultaneous eigenvalues of ad $\mathfrak{a}_{\mathfrak{p}}$ on $\mathfrak{g}$ as restricted roots. These are the nonzero restrictions to $\mathfrak{a}_{\mathfrak{p}}$ of the roots. Typical restricted roots are denoted by $\varepsilon$ or $\eta$, except that, in the case of the restriction to $\mathfrak{a}_{\mathfrak{p}}$ of a real root, we may denote the restricted root and the root by the same letter if there is no possibility of confusion. The set of restricted roots will be denoted by $\Sigma$. It is a root system, possibly not reduced.

For each restricted root $\varepsilon$, let $H_{\varepsilon}$ be the element of $\mathfrak{a}_{\mathfrak{p}}$ such that $\varepsilon(H)=S\left(H, H_{\varepsilon}\right)$ for all $H$ in $\mathfrak{a}_{\mathfrak{p}}, S$ being our invariant bilinear form. Since $G$ is linear, it makes sense to define

$$
\gamma_{\varepsilon}=\exp 2 \pi i|\varepsilon|^{-2} H_{\varepsilon}
$$

In the special case that $\varepsilon=\left.\alpha\right|_{\mathfrak{a}_{\mathfrak{p}}}$ for a real root $\alpha$, this definition is consistent with the one in (2.1). It is known that each element $\gamma_{\varepsilon}$ lies in $M_{\mathfrak{p}}$ and has order at most 2. It follows from (2.3) that $\left(M_{\mathfrak{p}}\right)_{0}$ and the elements $\gamma_{\varepsilon}$ together generate $M_{\mathfrak{p}}$.

If $\varepsilon$ is a restricted root, we write $s_{\varepsilon}$ for the Weyl group reflection in $\varepsilon$. This notation is sufficiently consistent with the definition in $\S 1$ of reflection in a real root that it will not cause any confusion. For two restricted roots $\varepsilon$ and $\eta$, direct computation from (4.1) gives

$$
\gamma_{s_{\eta} \varepsilon}=\gamma_{\varepsilon} \gamma_{\eta}^{2\langle\varepsilon, \eta\rangle /|\varepsilon|^{2}}
$$

For the most part we shall use (4.2) in the following form: If $\mathfrak{g}$ is simple and is not of type $G_{2}$, then

$$
\gamma_{s_{\eta} \varepsilon}= \begin{cases}\gamma_{\varepsilon} \gamma_{\eta} & \text { if }\langle\varepsilon, \eta\rangle \neq 0, \quad \eta \notin \mathbb{R} \varepsilon, \text { and }|\varepsilon| \geq|\eta|, \\ \gamma_{\varepsilon} & \text { if }\langle\varepsilon, \eta\rangle \neq 0, \quad \eta \notin \mathbb{R} \varepsilon, \text { and }|\varepsilon|<|\eta|\end{cases}
$$

Let $\varepsilon$ be a restricted root, and let $m(\varepsilon)$ be the sum of the multiplicities of $c \varepsilon$ as a restricted root for all $c>0$. If $m(\varepsilon)>1$, then Kn1 or Corollary 7.69 of Kn3] shows that $\gamma_{\varepsilon}$ is in $\left(M_{\mathfrak{p}}\right)_{0}$. Hence $M_{\mathfrak{p}}$ is generated by $\left(M_{\mathfrak{p}}\right)_{0}$ and all the $\gamma_{\varepsilon}$ for which $m(\varepsilon)=1$. These latter $\varepsilon$ 's are the restrictions to $\mathfrak{a}_{\mathfrak{p}}$ of real roots.

If positive systems of roots and restricted roots are specified, the positive systems are compatible if every root that restricts to a positive restricted root is a positive root. One way to obtain compatible systems is to define positivity relative to a lexicographic ordering of $\mathfrak{a}_{\mathfrak{p}}^{\prime} \oplus i \mathfrak{b}_{\mathfrak{p}}^{\prime}$ in which every positive element of $\mathfrak{a}_{\mathfrak{p}}^{\prime}$ is larger than every element of $i \mathfrak{b}_{\mathfrak{p}}^{\prime}$. For example, one could choose a basis of $\mathfrak{a}_{\mathfrak{p}}^{\prime}$, adjoin a basis of $i \mathfrak{b}_{\mathfrak{p}}^{\prime}$, and form the corresponding lexicographic orderings.

If compatible positive systems are imposed on the restricted roots and the roots, then any restricted root $\varepsilon$ for which $m(\varepsilon)=1$ is conjugate via a member of $W\left(\mathfrak{a}_{\mathfrak{p}}: K\right)$ to a simple restricted root $\varepsilon^{\prime}$ with $m\left(\varepsilon^{\prime}\right)=1$. Applying (4.2), we readily see that $M_{\mathfrak{p}}$ is generated by $\left(M_{\mathfrak{p}}\right)_{0}$ and all the $\gamma_{\varepsilon}$ for which $\varepsilon$ is a simple restricted root with $m(\varepsilon)=1$.

Satake $\mathrm{Sa}$ ] found relationships between simple roots and simple restricted roots in the setting of compatible positive systems. In part he proved that any simple 
restricted root is the restriction to $\mathfrak{a}_{\mathfrak{p}}$ of a simple root, and conversely the restriction to $\mathfrak{a}_{\mathfrak{p}}$ of a simple root is 0 or is a simple restricted root. In more detail the simple roots that are real restrict to simple restricted roots, the complex roots that are simple occur in pairs that restrict to the same simple restricted root, and the restrictions obtained in this way are linearly independent and exhaust the simple restricted roots. These results in part limit the possibilities for the quotient $M_{\mathfrak{p}} /\left(M_{\mathfrak{p}}\right)_{0}$. By contrast Theorem 2.1 of Wallach Wa1 limits the flexibility in defining finite-dimensional representations of $G$ in terms of the flexibility in defining representations of $M_{\mathfrak{p}}$. Implicit in the results of [Sa] and Wa1] together is the extent to which $\left(M_{\mathfrak{p}}\right)_{0}$ and the above elements $\gamma_{\varepsilon}$ are independent of one another. We make the resulting structure theorem explicit in Proposition 4.2 below, writing it in the form that will be useful to us. Proposition 4.2 uses Theorem 2.1 of Wa1] only as a pointer toward the method of proof, and thus [Wa1] does not need to be cited in the proof given below.

Lemma 4.1 (Satake). Fix compatible positive systems for $\Delta\left(\mathfrak{g}^{\mathbb{C}}, \mathfrak{h}_{\mathfrak{p}}^{\mathbb{C}}\right)$ and the restricted roots. If $\beta$ is a simple root that vanishes on $\mathfrak{b}_{\mathfrak{p}}$, then the restricted root $\varepsilon=\left.\beta\right|_{\mathfrak{a}_{\mathfrak{p}}}$ has $m(\varepsilon)=1$.

Proof. By one of the Satake results quoted above, $\varepsilon$ is a simple restricted root. On the other hand, we know from Kn1 that the only positive multiples of $\varepsilon$ that can be restricted roots are 1 and $\frac{1}{2}$. Since $\varepsilon$ is simple, the equation $\varepsilon=\frac{1}{2} \varepsilon+\frac{1}{2} \varepsilon$ shows that $\frac{1}{2} \varepsilon$ cannot be a restricted root.

Arguing by contradiction, suppose that $m(\varepsilon)>1$. By what we have just seen, some pair $\alpha^{ \pm}=\varepsilon \pm \omega$ consists of positive roots, with $\omega$ a nonzero element carried on $i \mathfrak{b}_{\mathfrak{p}}$. Then $\omega$ must be a root, and there is no loss of generality in assuming that it is positive. The formula $\varepsilon=\alpha^{-}+\omega$ exhibits $\varepsilon$ as the sum of positive roots and contradicts the fact that $\varepsilon$ was assumed simple. Hence $m(\varepsilon)=1$.

Proposition 4.2 (Satake-Wallach). Suppose that G has a simply connected complexification. Fix compatible positive systems for $\Delta\left(\mathfrak{g}^{\mathbb{C}}, \mathfrak{h}_{\mathfrak{p}}^{\mathbb{C}}\right)$ and the restricted roots. Then there exists a unique 1-dimensional representation $\sigma$ of $M_{\mathfrak{p}}$ such that $\sigma$ is 1 on the identity component $\left(M_{\mathfrak{p}}\right)_{0}$ and $\sigma\left(\gamma_{\varepsilon}\right)$ equals -1 for every simple restricted root with $m(\varepsilon)=1$.

Remark. If $\operatorname{rank} G=\operatorname{rank} K$, then the hypothesis that $G$ has a simply connected complexification implies that $G$ is acceptable in the sense of Harish-Chandra.

Proof. Uniqueness is immediate since $M_{\mathfrak{p}}$ is generated by $\left(M_{\mathfrak{p}}\right)_{0}$ and the elements $\gamma_{\varepsilon}$ for all simple restricted roots $\varepsilon$ with $m(\varepsilon)=1$. For existence define a highest weight $\lambda$ on $\mathfrak{h}_{\mathfrak{p}}^{\mathbb{C}}$ by the formula

$$
\frac{2\langle\lambda, \alpha\rangle}{|\alpha|^{2}}= \begin{cases}1 & \text { if } \alpha \text { is a simple root and is real, } \\ 0 & \text { if } \alpha \text { is a simple root and is nonreal. }\end{cases}
$$

Since $G$ has a simply connected complexification, there exists an irreducible representation $\pi_{\lambda}$ of $G$ with highest weight $\lambda$. When the underlying complex vector space $V$ is decomposed into restricted weight spaces, the highest restricted weight is $\left.\lambda\right|_{\mathfrak{a}_{\mathfrak{p}}}$, and we let $V_{1}$ be the corresponding weight space. The subgroup $M_{\mathfrak{p}}$ leaves $V_{1}$ stable, and it is well known (Lemma 8.48 of [Kn3]) that $\left(M_{\mathfrak{p}}\right)_{0}$ acts irreducibly in it. Let $\sigma$ be the representation of $M_{\mathfrak{p}}$ in this space. The highest weight of $\sigma$ is 
$\left.\lambda\right|_{\mathfrak{b}_{\mathfrak{p}}}$. From the structure of the simple roots, the linear span of the nonreal simple roots contains the dual of $\mathfrak{b}_{\mathfrak{p}}$. Since $\lambda$ is orthogonal to each nonreal simple root, $\lambda$ vanishes on $\mathfrak{b}_{\mathfrak{p}}$. Thus $\left.\lambda\right|_{\mathfrak{b}_{\mathfrak{p}}}=0$, and $\sigma$ is trivial on $\left(M_{\mathfrak{p}}\right)_{0}$. If $\alpha$ is real and simple, then $\sigma\left(\gamma_{\alpha}\right)=\exp \left(\lambda\left(2 \pi i H_{\alpha} /|\alpha|^{2}\right)\right)=\exp \left(\pi i\left(2\langle\lambda, \alpha\rangle /|\alpha|^{2}\right)\right)=\exp (\pi i)=-1$.

Proposition 4.3. Suppose that $G$ is quasisplit and that $\mathfrak{g}$ has a compact Cartan subalgebra $\mathfrak{t} \subseteq \mathfrak{k}$. Fix compatible positive systems for $\Delta\left(\mathfrak{g}^{\mathbb{C}}, \mathfrak{h}_{\mathfrak{p}}^{\mathbb{C}}\right)$ and the restricted roots. Suppose that $\alpha_{1}, \ldots, \alpha_{l}$ is a strongly orthogonal sequence of real roots whose restrictions to $\mathfrak{a}_{\mathfrak{p}}$ span $\mathfrak{a}_{\mathfrak{p}}^{\prime}$ and that $\Delta^{+}$coincides with the unique positive system of roots determined by lexicographic use of $\alpha_{1}, \ldots, \alpha_{l}$. For $1 \leq j \leq l$, let $\rho_{\alpha_{j}}$ be half the sum of the positive roots that are orthogonal to $\alpha_{1}, \ldots, \alpha_{j-1}$ but not to $\alpha_{j}$. If $\sigma$ is a 1-dimensional representation of $M_{\mathfrak{p}}$ such that $\sigma$ is 1 on the identity component $\left(M_{\mathfrak{p}}\right)_{0}$ and $\sigma\left(\gamma_{\varepsilon}\right)$ equals -1 for every simple restricted root with $m(\varepsilon)=1$, then

$$
\sigma\left(\gamma_{\alpha_{j}}\right)=(-1)^{2\left\langle\rho_{\alpha_{j}}, \alpha_{j}\right\rangle /\left|\alpha_{j}\right|^{2}} \quad \text { for } 1 \leq j \leq l .
$$

Remarks. Proposition 3.2 notes that lexicographic use of $\alpha_{1}, \ldots, \alpha_{l}$ determines a unique positive system of roots. Proposition 4.2 proves that the representation $\sigma$ in the current proposition exists if $G$ has a simply connected complexification. The quantity $\rho_{\alpha_{j}}$ is necessarily a positive multiple of $\alpha_{j}$ since there are no imaginary roots and the set of roots contributing to $\rho_{\alpha_{j}}$ is closed under each of $s_{\alpha_{j+1}}, \ldots, s_{\alpha_{l}}$.

Proof. In proving the displayed formula, we may assume that $\mathfrak{g}$ is simple. Assume temporarily that $\mathfrak{g}$ is not split $G_{2}$. Let $\delta$ be half the sum of the members of $\Delta^{+}=$ $\Delta^{+}\left(\mathfrak{g}^{\mathbb{C}}, \mathfrak{h}_{\mathfrak{p}}^{\mathbb{C}}\right)$. The particular ordering that we have chosen makes $\delta=\sum_{j=1}^{l} \rho_{\alpha_{j}}$; there is no contribution from $\mathfrak{b}_{\mathfrak{p}}^{\prime}$ because there are no imaginary roots and the positive complex roots are closed under $-\theta$. From this equation we obtain

$$
\frac{2\left\langle\delta, \alpha_{j}\right\rangle}{\left|\alpha_{j}\right|^{2}}=\frac{2\left\langle\rho_{\alpha_{j}}, \alpha_{j}\right\rangle}{\left|\alpha_{j}\right|^{2}}
$$

Therefore use of (3.7) shows that any simple root $\beta$ satisfies

$$
1=\frac{2\langle\delta, \beta\rangle}{|\beta|^{2}}=\sum_{j=1}^{l} \frac{2\left\langle\delta, \alpha_{j}\right\rangle}{|\beta|^{2}} \frac{\left\langle\beta, \alpha_{j}\right\rangle}{\left|\alpha_{j}\right|^{2}}=\sum_{j=1}^{l} \frac{2\left\langle\rho_{\alpha_{j}}, \alpha_{j}\right\rangle}{\left|\alpha_{j}\right|^{2}} \frac{\left\langle\beta, \alpha_{j}\right\rangle}{|\beta|^{2}}
$$

The given lexicographic ordering determines by restriction a set $\Sigma^{+}$of positive restricted roots. For fixed $k$ with $0 \leq k \leq l$, the set of restricted roots orthogonal to $\alpha_{1}, \ldots, \alpha_{k}$ is a root subsystem $\Sigma_{k}$, and any member of $\Sigma$ whose expansion in terms of $\alpha_{1}, \ldots, \alpha_{l}$ begins with a positive multiple of $\alpha_{i}$ for some $i \leq k$ is greater, in the lexicographic ordering, than every member of $\Sigma_{k}$. It follows that the restricted roots that are simple in $\Sigma^{+}$and are needed for the expansion of a member of $\Sigma_{k}$ all lie in $\Sigma_{k}$. Consequently, for $0 \leq k \leq l-1$, the expansion of $\alpha_{k+1}$ in terms of simple restricted roots involves some member of $\Sigma_{k}$ that is not in $\Sigma_{k+1}$.

We shall prove the displayed formula asserted in the proposition by induction downward on $j$ for $1 \leq j \leq l$. The base case of the induction is the empty statement that the assertion is true for indices beyond $l$. Assume inductively that the assertion in the displayed formula is true for indices $i+1$ up through $l$. By the argument in the previous paragraph, let $\varepsilon$ be a simple restricted root whose expansion in terms of $\alpha_{1}, \ldots, \alpha_{l}$ begins with a positive multiple of $\alpha_{i}$. From Satake [Sa], there is some simple $\operatorname{root} \beta$ such that $\varepsilon=\left.\beta\right|_{\alpha_{\mathfrak{p}}}$. 
According to Proposition 3.2, the expansion of $\beta$ in terms of simple roots begins with $\alpha_{i}$ or $\frac{1}{2} \alpha_{i}$. That proposition gives us seven possibilities for the expansion of $\beta$, and we look at each one in turn. One is that $\beta=\alpha_{i}$, the next four are that $\beta$ is real and is as in one of the formulas (3.1) through (3.4), and the last two are that $\beta$ is complex and is as in (3.5) or (3.6). In any event the fact that $\beta$ is simple means that the coefficients of any of $\alpha_{r}, \alpha_{s}$, and $\alpha_{t}$ that occur in the relevant formula (3.1) through (3.6) are negative.

If $\beta=\alpha_{i}$, then Lemma 4.1 says that $\beta=\alpha_{i}$, regarded as a restricted root, has $m(\beta)=1$. By definition we have $\sigma\left(\gamma_{\beta}\right)=-1$. Meanwhile $1=2\left\langle\delta, \alpha_{i}\right\rangle /\left|\alpha_{i}\right|^{2}=$ $2\left\langle\rho_{\alpha_{i}}, \alpha_{i}\right\rangle /\left|\alpha_{i}\right|^{2}$ by (4.4), and thus $(-1)^{2\left\langle\rho_{\alpha_{i}}, \alpha_{i}\right\rangle /\left|\alpha_{i}\right|^{2}}=-1$. Therefore $\sigma\left(\gamma_{\alpha_{i}}\right)=$ $(-1)^{2\left\langle\rho_{\alpha_{i}}, \alpha_{i}\right\rangle /\left|\alpha_{i}\right|^{2}}$ as required, and the inductive step is complete if $\beta=\alpha_{i}$.

If $\beta$ is as in (3.1), then $\beta=\frac{1}{2} \alpha_{i}-\frac{1}{2} \alpha_{r}-\frac{1}{2} \alpha_{s}-\frac{1}{2} \alpha_{t}$ with all the relevant root lengths equal. Hence $-s_{\alpha_{i}} \beta=s_{\alpha_{r}} s_{\alpha_{s}} s_{\alpha_{t}} \beta$, and (4.3) yields $\gamma_{\alpha_{i}} \gamma_{\beta}=\gamma_{\alpha_{t}} \gamma_{\alpha_{s}} \gamma_{\alpha_{r}} \gamma_{\beta}$. This formula and induction give

$$
\begin{aligned}
\sigma\left(\gamma_{\alpha_{i}}\right) & =\sigma\left(\gamma_{\alpha_{t}} \gamma_{\alpha_{s}} \gamma_{\alpha_{r}}\right) \\
& =(-1)^{2\left\langle\rho_{\alpha_{t}}, \alpha_{t}\right\rangle /\left|\alpha_{t}\right|^{2}}(-1)^{2\left\langle\rho_{\alpha_{s}}, \alpha_{s}\right\rangle /\left|\alpha_{s}\right|^{2}}(-1)^{2\left\langle\rho_{\alpha_{r}}, \alpha_{r}\right\rangle /\left|\alpha_{r}\right|^{2}} .
\end{aligned}
$$

On the other hand, (4.5) and the equality of all the lengths give

$$
1=\frac{1}{2} \frac{2\left\langle\rho_{\alpha_{i}}, \alpha_{i}\right\rangle}{\left|\alpha_{i}\right|^{2}}-\frac{1}{2} \frac{2\left\langle\rho_{\alpha_{r}}, \alpha_{r}\right\rangle}{\left|\alpha_{r}\right|^{2}}-\frac{1}{2} \frac{2\left\langle\rho_{\alpha_{s}}, \alpha_{s}\right\rangle}{\left|\alpha_{s}\right|^{2}}-\frac{1}{2} \frac{2\left\langle\rho_{\alpha_{t}}, \alpha_{t}\right\rangle}{\left|\alpha_{t}\right|^{2}} .
$$

Multiplying (4.7) by 2, using each side as an exponent of $(-1)$, and substituting from (4.6), we obtain

$$
\begin{aligned}
+1 & =(-1)^{2\left\langle\rho_{\alpha_{i}}, \alpha_{i}\right\rangle /\left|\alpha_{i}\right|^{2}}(-1)^{2\left\langle\rho_{\alpha_{r}}, \alpha_{r}\right\rangle /\left|\alpha_{r}\right|^{2}}(-1)^{2\left\langle\rho_{\alpha_{s}}, \alpha_{s}\right\rangle /\left|\alpha_{s}\right|^{2}}(-1)^{2\left\langle\rho_{\alpha_{t}}, \alpha_{t}\right\rangle /\left|\alpha_{t}\right|^{2}} \\
& =(-1)^{2\left\langle\rho_{\alpha_{i}}, \alpha_{i}\right\rangle /\left|\alpha_{i}\right|^{2}} \sigma\left(\gamma_{\alpha_{i}}\right) .
\end{aligned}
$$

This completes the inductive step if $\beta$ is as in (3.1).

If $\beta$ is as in (3.2), then $\beta=\frac{1}{2} \alpha_{i}-\alpha_{r}-\frac{1}{2} \alpha_{s}$ with $|\beta|=\left|\alpha_{i}\right|=\left|\alpha_{s}\right|>\left|\alpha_{r}\right|$. Hence $-s_{\alpha_{i}} \beta=s_{\alpha_{r}} s_{\alpha_{s}} \beta$, and (4.3) yields $\gamma_{\alpha_{i}} \gamma_{\beta}=\gamma_{\alpha_{s}} \gamma_{\alpha_{r}} \gamma_{\beta}$. This formula and induction give

$$
\sigma\left(\gamma_{\alpha_{i}}\right)=\sigma\left(\gamma_{\alpha_{s}} \gamma_{\alpha_{r}}\right)=(-1)^{2\left\langle\rho_{\alpha_{s}}, \alpha_{s}\right\rangle /\left|\alpha_{s}\right|^{2}}(-1)^{2\left\langle\rho_{\alpha_{r}}, \alpha_{r}\right\rangle /\left|\alpha_{r}\right|^{2}} .
$$

On the other hand, (4.5) and the relationships among the lengths give

$$
1=\frac{1}{2} \frac{2\left\langle\rho_{\alpha_{i}}, \alpha_{i}\right\rangle}{\left|\alpha_{i}\right|^{2}}-\frac{1}{2} \frac{2\left\langle\rho_{\alpha_{r}}, \alpha_{r}\right\rangle}{\left|\alpha_{r}\right|^{2}}-\frac{1}{2} \frac{2\left\langle\rho_{\alpha_{s}}, \alpha_{s}\right\rangle}{\left|\alpha_{s}\right|^{2}} .
$$

Multiplying (4.9) by 2 , using each side as an exponent of $(-1)$, and substituting from (4.8), we obtain

$$
\begin{aligned}
+1 & =(-1)^{2\left\langle\rho_{\alpha_{i}}, \alpha_{i}\right\rangle /\left|\alpha_{i}\right|^{2}}(-1)^{2\left\langle\rho_{\alpha_{r}}, \alpha_{r}\right\rangle /\left|\alpha_{r}\right|^{2}}(-1)^{2\left\langle\rho_{\alpha_{s}}, \alpha_{s}\right\rangle /\left|\alpha_{s}\right|^{2}} \\
& =(-1)^{2\left\langle\rho_{\alpha_{i}}, \alpha_{i}\right\rangle /\left|\alpha_{i}\right|^{2}} \sigma\left(\gamma_{\alpha_{i}}\right) .
\end{aligned}
$$

This completes the inductive step if $\beta$ is as in (3.2).

If $\beta$ is as in (3.3), then $\beta=\alpha_{i}-\frac{1}{2} \alpha_{r}-\frac{1}{2} \alpha_{s}$ with $|\beta|=\left|\alpha_{r}\right|=\left|\alpha_{s}\right|>\left|\alpha_{i}\right|$. Hence $-s_{\alpha_{i}} \beta=s_{\alpha_{r}} s_{\alpha_{s}} \beta$, and (4.3) yields $\gamma_{\alpha_{i}} \gamma_{\beta}=\gamma_{\alpha_{s}} \gamma_{\alpha_{r}} \gamma_{\beta}$. This formula and induction give the same result as in (4.8). On the other hand, (4.5) and the relationships among the lengths give the same result as in (4.9). Thus (4.10) again follows, and the inductive step is complete if $\beta$ is as in (3.3). 
If $\beta$ is as in (3.4), then $\beta=\frac{1}{2} \alpha_{i}-\frac{1}{2} \alpha_{r}$ with $|\beta|<\left|\alpha_{r}\right|=\left|\alpha_{i}\right|$. Hence $s_{\beta} \alpha_{i}=$ $\alpha_{i}-\frac{2\left\langle\alpha_{i}, \beta\right\rangle}{|\beta|^{2}} \beta=\alpha_{i}-2 \beta=\alpha_{i}-\left(\alpha_{i}-\alpha_{r}\right)=\alpha_{r}$, and (4.3) yields $\gamma_{\beta} \gamma_{\alpha_{i}}=\gamma_{\alpha_{r}}$. Also $\sigma\left(\gamma_{\beta}\right)=-1$ by Lemma 4.1. These formulas and induction give

$$
\sigma\left(\gamma_{\alpha_{i}}\right)=\sigma\left(\gamma_{\beta} \gamma_{\alpha_{r}}\right)=-(-1)^{2\left\langle\rho_{\alpha_{r}}, \alpha_{r}\right\rangle /\left|\alpha_{r}\right|^{2}} .
$$

On the other hand, (4.5) and the relationships among the lengths give

$$
1=\frac{2\left\langle\rho_{\alpha_{i}}, \alpha_{i}\right\rangle}{\left|\alpha_{i}\right|^{2}}-\frac{2\left\langle\rho_{\alpha_{r}}, \alpha_{r}\right\rangle}{\left|\alpha_{r}\right|^{2}}
$$

Using each side as an exponent of $(-1)$ and substituting from (4.11), we obtain

$$
-1=(-1)^{2\left\langle\rho_{\alpha_{i}}, \alpha_{i}\right\rangle /\left|\alpha_{i}\right|^{2}}(-1)^{2\left\langle\rho_{\alpha_{r}}, \alpha_{r}\right\rangle /\left|\alpha_{r}\right|^{2}}=-(-1)^{2\left\langle\rho_{\alpha_{i}}, \alpha_{i}\right\rangle /\left|\alpha_{i}\right|^{2}} \sigma\left(\gamma_{\alpha_{i}}\right) .
$$

This completes the inductive step if $\beta$ is as in (3.4).

If $\beta$ is as in (3.5), then $\beta=\frac{1}{2} \alpha_{i}+\omega$, and (4.5) gives $2\left\langle\rho_{\alpha_{i}}, \alpha_{i}\right\rangle /\left|\alpha_{i}\right|^{2}=2$. Hence $(-1)^{2\left\langle\rho_{\alpha_{i}}, \alpha_{i}\right\rangle /\left|\alpha_{i}\right|^{2}}=+1$. On the other hand, the roots contributing to $m\left(\alpha_{i}\right)$ upon restriction to $\mathfrak{a}_{\mathfrak{p}}$ include $\frac{1}{2} \alpha_{i}+\omega, \frac{1}{2} \alpha_{i}-\omega$, and $\alpha_{i}$. Hence $\gamma_{\alpha_{i}}$ lies in $\left(M_{\mathfrak{p}}\right)_{0}$ and $\sigma\left(\gamma_{\alpha_{i}}\right)=+1$. Therefore $\sigma\left(\gamma_{\alpha_{i}}\right)=(-1)^{2\left\langle\rho_{\alpha_{i}}, \alpha_{i}\right\rangle /\left|\alpha_{i}\right|^{2}}$, and the inductive step is complete if $\beta$ is as in (3.5).

If $\beta$ is as in (3.6), then $\beta=\frac{1}{2} \alpha_{i}-\frac{1}{2} \alpha_{r}+\omega$. Put $\varepsilon=\frac{1}{2} \alpha_{i}-\frac{1}{2} \alpha_{r}$. Since $\varepsilon+\omega$ and $\varepsilon-\omega$ both yield $\varepsilon$ upon restriction to $\mathfrak{a}_{\mathfrak{p}}, \gamma_{\varepsilon}$ lies in $\left(M_{\mathfrak{p}}\right)_{0}$ and $\sigma\left(\gamma_{\varepsilon}\right)=+1$. Computation gives $s_{\varepsilon}\left(\alpha_{r}\right)=\alpha_{i}$, and hence $\gamma_{\varepsilon} \gamma_{\alpha_{r}}=\gamma_{\alpha_{i}}$. Therefore $(+1) \sigma\left(\gamma_{\alpha_{r}}\right)=$ $\sigma\left(\gamma_{\varepsilon}\right) \sigma\left(\gamma_{\alpha_{r}}\right)=\sigma\left(\gamma_{\alpha_{i}}\right)$. This formula and induction give

$$
\sigma\left(\gamma_{\alpha_{i}}\right)=\sigma\left(\gamma_{\alpha_{r}}\right)=(-1)^{2\left\langle\rho_{\alpha_{r}}, \alpha_{r}\right\rangle /\left|\alpha_{r}\right|^{2}} .
$$

Meanwhile (4.5) gives

$$
1=\frac{1}{2} \frac{2\left\langle\rho_{\alpha_{i}}, \alpha_{i}\right\rangle}{\left|\alpha_{i}\right|^{2}}-\frac{1}{2} \frac{2\left\langle\rho_{\alpha_{r}}, \alpha_{i}\right\rangle}{\left|\alpha_{r}\right|^{2}} .
$$

Multiplying by 2 , using each side as an exponent of $(-1)$, and substituting from (4.13), we obtain

$$
+1=(-1)^{2\left\langle\rho_{\alpha_{i}}, \alpha_{i}\right\rangle /\left|\alpha_{i}\right|^{2}}(-1)^{2\left\langle\rho_{\alpha_{r}}, \alpha_{r}\right\rangle /\left|\alpha_{r}\right|^{2}}=(-1)^{2\left\langle\rho_{\alpha_{i}}, \alpha_{i}\right\rangle /\left|\alpha_{i}\right|^{2}} \sigma\left(\gamma_{\alpha_{i}}\right) .
$$

This completes the inductive step if $\beta$ is as in (3.6).

To complete the proof of Proposition 4.3, we must verify the proposition for split $G_{2}$. Roots and restricted roots are the same for this group since it is split. There are two cases for the sequence $\left\{\alpha_{1}, \alpha_{2}\right\}$. One is that $\alpha_{1}$ is long and $\alpha_{2}$ is short. In this case we find that

$$
\frac{2\left\langle\rho_{\alpha_{1}}, \alpha_{1}\right\rangle}{\left|\alpha_{1}\right|^{2}}=3 \quad \text { and } \quad \frac{2\left\langle\rho_{\alpha_{2}}, \alpha_{2}\right\rangle}{\left|\alpha_{2}\right|^{2}}=1
$$

so that

$$
(-1)^{2\left\langle\rho_{\alpha_{1}}, \alpha_{1}\right\rangle /\left|\alpha_{1}\right|^{2}}=(-1)^{2\left\langle\rho_{\alpha_{2}}, \alpha_{2}\right\rangle /\left|\alpha_{2}\right|^{2}}=-1 .
$$

The simple roots are $\alpha_{2}$ and $\beta=\frac{1}{2} \alpha_{1}-\frac{3}{2} \alpha_{2}$. Then $\sigma\left(\gamma_{\alpha_{2}}\right)=-1$ by definition. Also we have $-s_{\alpha_{1}} \beta=\frac{1}{2} \alpha_{1}+\frac{3}{2} \alpha_{2}=s_{\alpha_{2}} \beta$. Thus (4.2) gives $\gamma_{\beta} \gamma_{\alpha_{1}}=\gamma_{\beta} \gamma_{\alpha_{2}}$, and hence

$$
\sigma\left(\gamma_{\alpha_{1}}\right)=\sigma\left(\gamma_{\alpha_{2}}\right)=-1 .
$$

Comparison of (4.14) and (4.15) completes the argument in this case. 
The other case is that $\alpha_{1}$ is short and $\alpha_{2}$ is long. In this case we find that

$$
\frac{2\left\langle\rho_{\alpha_{1}}, \alpha_{1}\right\rangle}{\left|\alpha_{1}\right|^{2}}=5 \quad \text { and } \quad \frac{2\left\langle\rho_{\alpha_{2}}, \alpha_{2}\right\rangle}{\left|\alpha_{2}\right|^{2}}=1,
$$

so that (4.14) is still valid. The simple roots are $\alpha_{2}$ and $\beta=\frac{1}{2} \alpha_{1}-\frac{1}{2} \alpha_{2}$. Then $\sigma\left(\gamma_{\alpha_{2}}\right)=-1$ by definition. We have $-s_{\alpha_{1}} \beta=\frac{1}{2} \alpha_{1}+\frac{1}{2} \alpha_{2}=s_{\alpha_{2}} \beta$. Thus (4.2) again gives $\gamma_{\beta} \gamma_{\alpha_{1}}=\gamma_{\beta} \gamma_{\alpha_{2}}$ and yields the consequence (4.15). Comparison of (4.14) and (4.15) again completes the argument.

\section{Sum of All totally DEgENERATE Limits OF DisCRETE SERIES}

In this section we show first that the unitary principal series representation corresponding to $\sigma$ on $M_{\mathfrak{p}}$ and the trivial character on $A_{\mathfrak{p}}$ is the sum of all the totally degenerate limits of discrete series if $\sigma$ is defined as in $\S 4$. This step is carried out in Theorems 5.1 and 5.2. It is automatic from Theorem 7.1 of [Kn2] that the multiplicities in this decomposition are all 1 . We might mention that it follows from Harish-Chandra's subquotient theorem and a little extra argument (which actually is contained in the proofs of Theorems 5.1 and 5.2 below) that there is some irreducible representation of $M_{\mathfrak{p}}$ with this property, but we are interested in isolating the possible choices for this representation and seeing that $\sigma$ is one of them.

The concrete information assembled in $\S 4$ about $\sigma$ allows us to do more. The main thing is that it allows us to apply the part of the theory of the $R$ group in $\mathrm{KnZ}$ to give concretely the classification parameters of the irreducible constituents of the corresponding principal series representation; these will be described in Theorem 5.5 and will be listed for each simple group in Table 5.1. Parenthetically it allows us also to see that totally degenerate limits of discrete series exist whenever $\sigma$ makes sense; thus $G$ has totally degenerate limits of discrete series if and only if $\operatorname{rank} G=\operatorname{rank} K$, $G$ is quasisplit, and $G$ is acceptable in the sense of Harish-Chandra (i.e., half the sum of the positive roots is analytically integral). We continue with notation as in $\S 4$.

Theorem 5.1. Let $G$ be quasisplit with rank $G=\operatorname{rank} K$, and suppose that $G$ is acceptable in the sense of Harish-Chandra. Fix a strongly orthogonal sequence $\alpha_{1}, \ldots, \alpha_{l}$ of real roots relative to $\mathfrak{h}_{\mathfrak{p}}$ whose restrictions to $\mathfrak{a}_{\mathfrak{p}}$ span $\mathfrak{a}_{\mathfrak{p}}^{\prime}$, and let $\Delta^{+}=\Delta^{+}\left(\mathfrak{g}^{\mathbb{C}}, \mathfrak{h}_{\mathfrak{p}}^{\mathbb{C}}\right)$ be the unique positive system determined by $\alpha_{1}, \ldots, \alpha_{l}$. Impose the relative ordering on restricted roots. Then there exists a unique 1-dimensional representation $\sigma$ of $M_{\mathfrak{p}}$ such that $\sigma$ is 1 on the identity component $\left(M_{\mathfrak{p}}\right)_{0}$ and $\sigma\left(\gamma_{\varepsilon}\right)$ equals -1 for every simple restricted root with $m(\varepsilon)=1$. If $N_{\mathfrak{p}}$ denotes the analytic subgroup of $G$ corresponding to the sum of the restricted-root spaces for the positive restricted roots and if $Q_{\mathfrak{p}}$ is $M_{\mathfrak{p}} A_{\mathfrak{p}} N_{\mathfrak{p}}$, then each irreducible constituent of $\operatorname{ind}_{Q_{\mathfrak{p}}}^{G}(\sigma \otimes 1 \otimes 1)$ is a totally degenerate limit of discrete series representation.

Remarks. Proposition 3.1 guarantees that $\alpha_{1}, \ldots, \alpha_{l}$ exists, and Proposition 3.2 guarantees that $\Delta^{+}$is uniquely determined by $\alpha_{1}, \ldots, \alpha_{l}$. Under the ordering, every positive element of $\mathfrak{a}_{\mathfrak{p}}^{\prime}$ is greater than every element of $i \mathfrak{b}_{\mathfrak{p}}^{\prime}$, and therefore $\Delta^{+}$ consistently defines a compatible notion of positivity for restricted roots.

Proof. Let us assume for the moment that $G$ has a simply connected complexification. The existence and uniqueness of $\sigma$ are then given by Proposition 4.2. 
Proposition 4.3 shows that

$$
\sigma\left(\gamma_{\alpha_{j}}\right)=(-1)^{2\left\langle\rho_{\alpha_{j}}, \alpha_{j}\right\rangle /\left|\alpha_{j}\right|^{2}}
$$

for $1 \leq j \leq l$, and we know that the global character of $\operatorname{ind}_{Q_{\mathfrak{p}}}^{G}(\sigma \otimes 1 \otimes 1)$ is of the form

$$
\operatorname{ind}_{Q_{\mathfrak{p}}}^{G} \Theta^{M_{\mathfrak{p}} A_{\mathfrak{p}}}\left(0, i \mathfrak{b}_{\mathfrak{p}},\left.\sigma\right|_{F\left(B_{\mathfrak{p}}\right)}, 0\right) .
$$

We wish to invert generalized Schmid identities relative to $\mathbf{d}_{\alpha_{l}}, \ldots, \mathbf{d}_{\alpha_{1}}$ so that (5.2) can be rewritten one step at a time in terms of data for a Cartan subalgebra whose compact part is one dimension larger. The condition for doing so is stated at the end of $\S 2$ and has two parts to it. One part concerns the $\mathfrak{a}_{\mathfrak{p}}$ parameter; since this parameter is 0 in our situation, it presents no difficulty. The other part concerns the values of $\left.\sigma\right|_{F\left(B_{\mathfrak{p}}\right)}\left(\gamma_{\alpha_{j}}\right)=\sigma\left(\gamma_{\alpha_{j}}\right)$ for $j=l, \ldots, 1$, and (5.1) says that this part of the condition is satisfied. Therefore we can rewrite (5.2) as the sum of one or two characters built from a Cartan subalgebra that incorporates $\widetilde{\alpha}_{l}=\mathbf{d}_{\alpha_{l}}\left(\alpha_{l}\right)$ as a noncompact imaginary root, and we can rewrite each of those as the sum of one or two characters from a Cartan subalgebra that also incorporates $\widetilde{\alpha}_{l-1}=\mathbf{d}_{\alpha_{l-1}}\left(\alpha_{l-1}\right)$ as a noncompact imaginary root. The imaginary root $\widetilde{\alpha_{l}}$ remains noncompact after $\mathbf{d}_{\alpha_{l-1}}$ because $\alpha_{l-1}$ and $\alpha_{l}$ are strongly orthogonal. We continue in this way through $\alpha_{l-1}, \ldots, \alpha_{1}$. At each step the data other than the chambers (and the implied sets of positive roots) are the same in all the new characters that arise, as was remarked at the end of $\S 2$. Since the restrictions of $\alpha_{l}, \ldots, \alpha_{1}$ span $\mathfrak{a}_{\mathfrak{p}}^{\prime}$, the final Cartan subalgebra is compact. Thus all the global characters after the last stage are limits of discrete series, and their infinitesimal character in each case is 0 . Some of these global characters may be 0 , but at least one of them is nonzero because (5.2) is nonzero. Each of the nonzero limits of discrete series is irreducible because nonzero limits of discrete series are always irreducible (Theorem 1.1 of [KnZ]). This completes the proof under the assumption that $G$ has a simply connected complexification.

Now suppose that the linear group $G$ is merely acceptable. Choose a covering group $\widetilde{G}$ with a simply connected complexification, and let $Z$ be the kernel of the covering map $\widetilde{G} \rightarrow G$. Apply the special case just considered to the group $\widetilde{G}$, obtaining a representation $\widetilde{\sigma}$ of the group $\widetilde{M}_{\mathfrak{p}}$. Let $\widetilde{Q}_{\mathfrak{p}}=\widetilde{M}_{\mathfrak{p}} A_{\mathfrak{p}} N_{\mathfrak{p}}$ be the minimal parabolic subgroup such that $\operatorname{ind}_{\widetilde{Q}_{\mathfrak{p}}}^{\widetilde{G}}(\widetilde{\sigma} \otimes 1 \otimes 1)$ is exhibited as a sum of totally degenerate limits of discrete series. Let any of the latter be $\widetilde{\pi}(0, C)$. Since $G$ is acceptable and 0 is algebraically integral, $\widetilde{\pi}(0, C)$ is trivial on $Z$. Therefore $\operatorname{ind}_{\widetilde{Q}_{\mathfrak{p}}}^{\widetilde{G}}(\widetilde{\sigma} \otimes 1 \otimes 1)$ is trivial on $Z$, and we conclude that $\widetilde{\sigma}$ is trivial on $Z$. Then $\widetilde{\sigma}$ descends to a representation $\sigma$ of $M_{\mathfrak{p}}$. This proves the existence of $\sigma$, and the uniqueness is immediate from the fact that $M_{\mathfrak{p}}$ is generated by $\left(M_{\mathfrak{p}}\right)_{0}$ and the elements $\gamma_{\varepsilon}$ for the simple restricted roots $\varepsilon$ with $m(\varepsilon)=1$. This completes the proof.

Theorem 5.2. Let $G$ be quasisplit with rank $G=\operatorname{rank} K$, and suppose that $G$ is acceptable in the sense of Harish-Chandra. Then $G$ has a totally degenerate limit of discrete series representation. Fix one of the representations of this kind that is produced by Theorem 5.1, and write its global character as $\Theta^{G}(0, C)$ relative to some compact Cartan subalgebra $\mathfrak{t}$ of $\mathfrak{g}$ contained in $\mathfrak{k}$. Let $U$ be the analytic subgroup of $G^{\mathbb{C}}$ with Lie algebra $\mathfrak{u}=\mathfrak{k} \oplus i \mathfrak{p}$. Then the global character of any totally degenerate 
limit of discrete series representation of $G$ is of the form $\Theta^{G}(0, \operatorname{Ad}(u) C)$ for some member $u$ of the normalizer $N_{U}(\mathfrak{t}, \mathfrak{k}, \mathfrak{p})$ of $\mathfrak{t}, \mathfrak{k}$, and $\mathfrak{p}$ in $U$. Moreover every totally degenerate limit of discrete series representation is a constituent of the unitary principal series representation $\operatorname{ind}_{Q_{\mathfrak{p}}}^{G}(\sigma \otimes 1 \otimes 1)$ of Theorem 5.1.

Remark. $U$ is a compact form of $G$.

Proof. It is immediate from Theorem 5.1 that $G$ has a totally degenerate limit of discrete series representation. We can write its global character as $\Theta^{G}(0, C)$. Since $\Theta^{G}(0, C)$ is nonzero, the Hecht-Schmid identity shows that every $C$-simple root relative to $\Delta\left(\mathfrak{g}^{\mathbb{C}}, \mathfrak{t}^{\mathbb{C}}\right)$ is noncompact. Any other totally degenerate limit of discrete series representation has a global character of the form $\Theta^{G}\left(0, C^{\prime}\right)$. The action of the Weyl group $W\left(\mathfrak{t}^{\mathbb{C}}: \mathfrak{g}^{\mathbb{C}}\right)$ is transitive on the set of chambers, and each Weyl group element has a representative in $U$. Thus we can choose $u \in U$ normalizing $\mathfrak{t}$ with $C^{\prime}=\operatorname{Ad}(u) C$. The transformation $\operatorname{Ad}(u)$ carries the $C$-simple roots to the $C^{\prime}$ simple roots, and every $C^{\prime}$-simple root is noncompact since $\Theta^{G}\left(0, C^{\prime}\right)$ is assumed nonzero. Thus $\operatorname{Ad}(u)$ preserves type - compact or noncompact - for simple roots. An easy induction allows us to extend this conclusion to all positive roots and to their negatives. For example, if the result is known for a compact root $\alpha$ and a noncompact root $\beta$, then the root vectors satisfy

$$
\operatorname{Ad}(u)\left[E_{\alpha}, E_{\beta}\right]=\left[\operatorname{Ad}(u) E_{\alpha}, \operatorname{Ad}(u) E_{\beta}\right] \subseteq\left[\mathfrak{k}^{\mathbb{C}}, \mathfrak{p}^{\mathbb{C}}\right] \subseteq \mathfrak{p}^{\mathbb{C}},
$$

so that $\operatorname{Ad}(u)$ carries the noncompact root $\alpha+\beta$ to a noncompact root. The conclusion is that $\operatorname{Ad}(u)$ preserves type for all roots. Consequently $\operatorname{Ad}(u)$ carries $\mathfrak{k}^{\mathbb{C}}$ to itself and $\mathfrak{p}^{\mathbb{C}}$ to itself. But also $\operatorname{Ad}(u)$ carries the Lie algebra $\mathfrak{u}=\mathfrak{k} \oplus i \mathfrak{p}$ to itself, and hence it carries the intersections $\mathfrak{k}=\mathfrak{u} \cap \mathfrak{k}^{\mathbb{C}}$ and $\mathfrak{p}=i \mathfrak{u} \cap \mathfrak{p}^{\mathbb{C}}$ to themselves. This proves that $u$ is in $N_{U}(\mathfrak{t}, \mathfrak{k}, \mathfrak{p})$.

If $\pi$ is a unitary representation of $G$ on a Hilbert space $H$ and if $\varphi$ is an automorphism of $G$, we define $\pi^{\varphi}$ to be the unitary representation of $G$ on $H$ given by $\pi^{\varphi}=\pi \circ \varphi^{-1}$. If $\varphi$ is an inner automorphism, then $\pi^{\varphi}$ will of course be unitarily equivalent with $\pi$.

Suppose now that $\pi$ is a representation with global character $\Theta^{G}(0, C)$, and let $\varphi(x)=u x u^{-1}$ for $x \in G$. The same argument that establishes (1.14) in [KnZ proves that $\pi^{\varphi}$ has global character $\Theta^{G}(0, \operatorname{Ad}(u) C)$.

From the embedding of $\pi$ into $L=\operatorname{ind}_{Q_{\mathfrak{p}}}^{G}(\sigma \otimes 1 \otimes 1)$, it follows that $\pi^{\varphi}$ embeds into $L^{\varphi}$. We shall adjust $\varphi$ by an inner automorphism to exhibit an equivalence between $L$ and $L^{\varphi}$. Specifically we forget about the fact that $u$ normalizes $\mathfrak{t}$ and remember only that $u$ is in $U$ and $u$ normalizes $\mathfrak{g}, \mathfrak{k}$, and $\mathfrak{p}$. The space $\operatorname{Ad}(u)\left(\mathfrak{a}_{\mathfrak{p}}\right)$ is maximal abelian in $\mathfrak{p}$, and we can find $k_{1} \in K$ so that $\operatorname{Ad}\left(k_{1} u\right)\left(\mathfrak{a}_{\mathfrak{p}}\right)=\mathfrak{a}_{\mathfrak{p}}$. The various possibilities for $\mathfrak{n}_{\mathfrak{p}}$ are conjugate via the Weyl group $W\left(\mathfrak{a}_{\mathfrak{p}}: K\right)$, and thus we can find $k_{2} \in K$ so that $\operatorname{Ad}\left(k_{2} k_{1} u\right)$ carries $\mathfrak{a}_{\mathfrak{p}}$ to itself and preserves positivity of simple restricted roots. Then conjugation by $k_{2} k_{1} u$ maps $M_{\mathfrak{p}}$ and $N_{\mathfrak{p}}$ to themselves. Let us put $v=k_{2} k_{1} u$ and redefine $\varphi$ by $\varphi(x)=v x v^{-1}$. For the redefined $\varphi$, we work with $L^{\varphi}=\left(\operatorname{ind}_{Q_{\mathfrak{p}}}^{G}(\sigma \otimes 1 \otimes 1)\right)^{\varphi}$. Let us say for definiteness that the induced representations act on the left, with the transformation law being on the right. We define an operator $P$ on the space of $L^{\varphi}$ (i.e., the space of $L$ ) by $F(x)=(P f)(x)=f\left(\varphi^{-1} x\right)=f\left(v^{-1} x v\right)$, and we readily check that $P$ carries the space of $L^{\varphi}$ in one-one fashion onto the space for $L^{\prime}=\operatorname{ind}_{Q_{\mathfrak{p}}}^{G}\left(\sigma^{\varphi} \otimes 1 \otimes 1\right)$ and that $P$ exhibits these representations as equivalent: $L^{\prime}(g)(P f)(x)=P\left(\left(L^{\varphi}(g)\right) f\right)(x)$. 
To show that $L^{\varphi}$ is equivalent with $L$, it is therefore enough to show that $\sigma^{\varphi}$ equals $\sigma$, i.e., that $\sigma\left(v^{-1} m v\right)=\sigma(m)$ for all $m \in M_{\mathfrak{p}}$. Conjugation of $M_{\mathfrak{p}}$ by $v^{-1}$ carries $\left(M_{\mathfrak{p}}\right)_{0}$ to itself, and $\sigma$ is 1 on $\left(M_{\mathfrak{p}}\right)_{0}$. Thus it is enough to show that $\sigma\left(v^{-1} \gamma_{\varepsilon} v\right)=\sigma\left(\gamma_{\varepsilon}\right)$ for every simple restricted root $\varepsilon$. Here $v^{-1} \gamma_{\varepsilon} v=\gamma_{\operatorname{Ad}\left(v^{-1}\right) \varepsilon}$, and we have arranged that conjugation by $v^{-1}$ maps the set of simple restricted roots to itself. It also preserves multiplicities. Thus if $\varepsilon$ is a simple restricted root with $m(\varepsilon)=1$, so is $\operatorname{Ad}\left(v^{-1}\right) \varepsilon$. The definition of $\sigma$ then shows that $\sigma$ is -1 on both $\gamma_{\varepsilon}$ and $\gamma_{\operatorname{Ad}\left(v^{-1}\right) \varepsilon}$. Hence $\sigma^{\varphi}=\sigma$.

What all this shows is that a representation with global character $\Theta^{G}\left(0, C^{\prime}\right)=$ $\Theta^{G}(0, \operatorname{Ad}(u) C)$ embeds in a representation equivalent with $\operatorname{ind}_{Q_{\mathfrak{p}}}^{G}(\sigma \otimes 1 \otimes 1)$, and the theorem is thus completely proved.

Corollary 5.3. Let $G$ be quasisplit with rank $G=\operatorname{rank} K$, and suppose that $G$ is acceptable in the sense of Harish-Chandra. Let $U$ be the analytic subgroup of $G^{\mathbb{C}}$ with Lie algebra $\mathfrak{u}=\mathfrak{k} \oplus$ ip. If $N_{U}(\mathfrak{t}, \mathfrak{k}, \mathfrak{p})$ denotes the subgroup of elements of $U$ normalizing $\mathfrak{t}, \mathfrak{k}$, and $\mathfrak{p}$, then the inclusions of $N_{U}(\mathfrak{t}, \mathfrak{k}, \mathfrak{p})$ first into $N_{U}(\mathfrak{k})$ and then into $N_{G^{\mathbb{C}}}(\mathfrak{g})$ descend to isomorphisms

$$
N_{U}(\mathfrak{t}, \mathfrak{k}, \mathfrak{p}) / N_{K}(\mathfrak{t}) \cong N_{U}(\mathfrak{k}) / K \cong N_{G^{\mathbb{C}}}(\mathfrak{g}) / G .
$$

Consequently the set of totally degenerate limits of discrete series characters of $G$ is parametrized by either of the groups $N_{U}(\mathfrak{k}) / K$ or $N_{G^{\mathbb{C}}}(\mathfrak{g}) / G$.

Proof. A member of $N_{U}(\mathfrak{k})$ normalizes $\mathfrak{k}$ and $\mathfrak{k} \oplus i \mathfrak{p}$. Hence it normalizes $i \mathfrak{p}$, $\mathfrak{p}$, and $\mathfrak{k} \oplus \mathfrak{p}=\mathfrak{g}$. Therefore $N_{U}(\mathfrak{t}, \mathfrak{k}, \mathfrak{p}) \subseteq N_{U}(\mathfrak{k}) \subseteq N_{G^{\mathbb{C}}}(\mathfrak{g})$. Since $N_{K}(\mathfrak{t}) \subseteq K \subseteq G$, the inclusions descend to homomorphisms

$$
N_{U}(\mathfrak{t}, \mathfrak{k}, \mathfrak{p}) / N_{K}(\mathfrak{t}) \rightarrow N_{U}(\mathfrak{k}) / K \rightarrow N_{G^{\mathbb{C}}}(\mathfrak{g}) / G .
$$

To see that the maps (5.4) are one-one, we have to see that $N_{U}(\mathfrak{t}, \mathfrak{k}, \mathfrak{p}) \cap G \subseteq N_{K}(\mathfrak{t})$. It is enough to see that $U \cap G \subseteq K$. Since $G=K \exp \mathfrak{p}$, it is enough to see that $U \cap \exp \mathfrak{p}=1$. If $X \neq 0$ is in $\mathfrak{p}$, then $\operatorname{Ad}(\exp X)$ acts fully reducibly with positive eigenvalues on $\mathfrak{g}^{\mathbb{C}}$, while any member of $\operatorname{Ad}(U)$ acts with all eigenvalues of modulus 1 since $U$ is compact. The two can coincide only if all the eigenvalues are 1 , and then $\operatorname{Ad}(\exp X)=1$, ad $X=0$, and $X=0$. Thus the maps (5.4) are one-one.

To see that the first map in (5.4) is onto, let $u$ be given in $N_{U}(\mathfrak{k})$. Then $\operatorname{Ad}(u)$ carries $\mathfrak{t}$ to a maximal abelian subspace of $\mathfrak{k}$, and we can find $k \in K$ such that $\operatorname{Ad}(k u)$ carries $\mathfrak{t}$ to itself. The element $k u$ normalizes $\mathfrak{t}, \mathfrak{k}$, and $\mathfrak{p}$. Then $k u$ is in $N_{U}(\mathfrak{t}, \mathfrak{k}, \mathfrak{p})$, and the $K$ coset of our original element $u$ is exhibited as in the image of the first map of (5.4).

To see that the second map in (5.4) is onto, let $g$ be given in $N_{G^{\mathrm{C}}}(\mathfrak{g})$. By the global Cartan decomposition of $G^{\mathbb{C}}$, write $g=u \exp Z$ with $u \in U$ and $Z \in i \mathfrak{u}$. The space $\mathfrak{g}$ is stable under the Cartan involution of $\mathfrak{g}^{\mathbb{C}}$, which is +1 on $\mathfrak{u}$ and -1 on $i \mathfrak{u}$, and thus Lemma 7.22 of $\mathrm{Kn} 3$ applies and shows that $u$ and $Z$ separately normalize $\mathfrak{g}$. Here $u$ is in $N_{U}(\mathfrak{k})$, which we have already observed is a subset of $N_{G^{\mathbb{C}}}(\mathfrak{g})$. Meanwhile, $Z$ is in $i \mathfrak{k} \oplus \mathfrak{p}$. Write $Z=Z_{1}+Z_{2}$ accordingly. Then $Z_{2}$ is in $\mathfrak{p} \subseteq \mathfrak{g}$ and hence normalizes $\mathfrak{g}$. So $Z_{1}$ normalizes $\mathfrak{g}$. Since $Z_{1}$ is in $i \mathfrak{g}$, we obtain $\left[Z_{1}, \mathfrak{g}\right] \subseteq i \mathfrak{g} \cap \mathfrak{g}=0$, and hence $i Z_{1}$ is in the center of $\mathfrak{g}$. This is 0 , and thus we conclude that $g=u z$ with $z=\exp Z_{2}$ in $G$. Thus our member $g$ of $N_{G^{\mathrm{C}}}(\mathfrak{g})$ is in the same $G$ coset as $u$, which we have seen is in $N_{U}(\mathfrak{k})$.

Now consider the last assertion of the corollary. From Theorem 5.2 we know that there is at least one such character, and it is of the form $\Theta^{G}(0, C)$ for some 
chamber $C$. Also we know that any other one is of the form $\Theta^{G}(0, \operatorname{Ad}(u) C)$ with $u$ in $N_{U}(\mathfrak{t}, \mathfrak{k}, \mathfrak{p})$. We want to know the isotropy subgroup, i.e., those elements $u \in N_{U}(\mathfrak{t}, \mathfrak{k}, \mathfrak{p})$ with $\Theta^{G}(0, \operatorname{Ad}(u) C)=\Theta^{G}(0, C)$. Theorem 1.1c of [KnZ gives a condition-that $\operatorname{Ad}(u) C=\operatorname{Ad}(g) C$ for some $g$ in $N_{G}(\mathfrak{t})$. Let $g=k \exp X$ be the global Cartan decomposition of $g$. Lemma 7.22 of [Kn3] shows that $k$ and $\exp X$ separately normalize $\mathfrak{t}$. Since $\mathfrak{t}$ is a Cartan subalgebra, it equals its own normalizer, and thus $X$ is in $\mathfrak{t}$. Hence $\operatorname{Ad}(u) C=\operatorname{Ad}(k) C$ for some $k$ in $N_{K}(\mathfrak{t})$. Since the Weyl group $W(\mathfrak{t}: U)$ acts simply transitively on the Weyl chambers, $k^{-1} u$ is in $\exp \mathfrak{t}$. Thus $u$ is in $N_{K}(\mathfrak{t})$. Consequently $N_{U}(\mathfrak{t}, \mathfrak{k}, \mathfrak{p}) / N_{K}(\mathfrak{t})$ parametrizes the set of characters of totally degenerate limits of discrete series of $G$, and the last assertion of the corollary follows from the isomorphisms (5.3).

The theory of the $R$ group in $\mathrm{KnZ}$ tells how to obtain the classification parameters of the irreducible constituents of $\operatorname{ind}_{Q_{\mathfrak{p}}}^{G}(\sigma \otimes 1 \otimes 1)$. To describe matters easily, let us assume that $G$ is simple, and let us take advantage of the fact that $\sigma$ is 1-dimensional. (For the general case, see p. 438 of [KnZ].) If $k \in K$ normalizes $\mathfrak{a}_{\mathfrak{p}}$, then we set $k \sigma(m)=\sigma\left(k^{-1} m k\right)$. Since $\sigma$ is 1-dimensional, the right side does not change when $k$ is multiplied by a member of the centralizer $M_{\mathfrak{p}}$ of $\mathfrak{a}_{\mathfrak{p}}$ in $K$. Thus it makes sense to to speak of $w \sigma$ for any $w$ in the Weyl group $W\left(\mathfrak{a}_{\mathfrak{p}}: K\right)$, which is the same as the Weyl group of the system $\Sigma$ of restricted roots. Let

$$
W_{\sigma}=\left\{w \in W\left(\mathfrak{a}_{\mathfrak{p}}: K\right) \mid w \sigma=\sigma\right\} .
$$

To get at the $R$ group, one defines a certain subset $\Sigma_{\sigma}^{\prime}$ of $\Sigma$. For 1-dimensional $\sigma$ when the irreducible root system $\Sigma$ is reduced,

$$
\Sigma_{\sigma}^{\prime}=\left\{\varepsilon \text { in } \Sigma \mid \sigma\left(\gamma_{\varepsilon}\right)=+1\right\} .
$$

We shall not need the definition of $\Sigma_{\sigma}^{\prime}$ when $\Sigma$ is not reduced. The reason is that $M_{\mathfrak{p}}$ is connected in this case (see [Kn1]), and $\sigma$ reduces to the trivial representation; the $R$ group is easily seen from its definition to be trivial in this case.

In any event, $\Sigma_{\sigma}^{\prime}$ is closed under its own reflections, and these reflections are in $W_{\sigma}$. It follows readily that $W_{\sigma}$ is the semidirect product of the Weyl group $W_{\sigma}^{\prime}$ of $\Sigma_{\sigma}^{\prime}$ and the subgroup

$$
R_{\sigma}=\left\{r \in W_{\sigma} \mid r \varepsilon>0 \text { for all } \varepsilon>0 \text { in } \Sigma_{\sigma}^{\prime}\right\} .
$$

Proposition 8.4 of $\left[\mathrm{KnZ}\right.$ says that there is a set $\mathcal{H}=\mathcal{H}_{\sigma}=\left\{\xi_{1}, \ldots, \xi_{p}\right\}$ of real roots in $\Delta^{+}\left(\mathfrak{g}^{\mathbb{C}}, \mathfrak{h}_{\mathfrak{p}}^{\mathbb{C}}\right)$ that are superorthogonal in the sense that no nontrivial linear combination of $\xi_{1}, \ldots, \xi_{p}$ is a root, every member $r$ of $R_{\sigma}$ is the product of the reflections in the restrictions to $\mathfrak{a}_{\mathfrak{p}}$ of the members of some subset of $\mathcal{H}$, and every member $\xi$ of $\mathcal{H}$ has the property that $s_{\xi}$ is a factor of some member of $R_{\sigma}$.

Table 5.1 tells what $\mathcal{H}$ is for all simple $G$ under study. In most cases the group $R_{\sigma}$ has order 1 or 2 . In the cases of order 2 , the nontrivial element of $R_{\sigma}$ is the product of the reflections in $\mathcal{H}$. The only case for which $R_{\sigma}$ has order greater than 2 is $D_{2 n}$, and then $R_{\sigma}$ has order 4; two of the nontrivial elements are

$$
s_{e_{2 n-1}-e_{2 n}} s_{e_{2 n-1}+e_{2 n}} \quad \text { and } \quad s_{e_{1}-e_{2}} s_{e_{3}-e_{4}} \cdots s_{e_{2 n-3}-e_{2 n-2}} s_{e_{2 n-1}-e_{2 n}},
$$

and the third is the product of these two. In $\S 6$ we shall need the following result, which is obvious by inspection from Table 5.1. 
Table 5.1. Set $\mathcal{H}_{\sigma}$ of real roots identified by $R_{\sigma}$

\begin{tabular}{|c|c|c|c|c|c|}
\hline$\Delta$ & $\bar{\Sigma}$ & $\begin{array}{l}\text { Names of Simple } \\
\text { Restricted Roots }\end{array}$ & $\begin{array}{c}m(\varepsilon) \text { for Each } \\
\text { Simple } \\
\text { Restricted Root }\end{array}$ & $\left|R_{\sigma}\right|$ & $\overline{\mathcal{H}_{\sigma}}$ \\
\hline$\overline{A_{2 n-1}}$ & $\overline{C_{n}}$ & $\begin{array}{c}e_{j}-e_{j+1} \\
1 \leq j \leq n-1 \\
2 e_{n}\end{array}$ & $\begin{array}{c}2 \text { for short } \varepsilon \\
1 \text { for long } \varepsilon\end{array}$ & 2 & $2 e_{n}$ \\
\hline$A_{2 n}$ & $(B C)_{n}$ & not needed & $\begin{array}{c}3 \text { for short } \varepsilon \\
2 \text { for medium } \varepsilon\end{array}$ & 1 & $\varnothing$ \\
\hline$B_{2 n}$ & $B_{2 n}$ & $\begin{array}{c}e_{j}-e_{j+1} \\
1 \leq j \leq 2 n-1 \\
e_{2 n}\end{array}$ & 1 for all $\varepsilon$ & 2 & $\begin{array}{c}e_{2 j-1}-e_{2 j} \\
1 \leq j \leq n\end{array}$ \\
\hline$B_{2 n+1}$ & $B_{2 n+1}$ & $\begin{array}{c}e_{j}-e_{j+1} \\
1 \leq j \leq 2 n \\
e_{2 n+1}\end{array}$ & 1 for all $\varepsilon$ & 2 & $\begin{array}{c}e_{2 j-1}-e_{2 j} \\
1 \leq j \leq n \\
\quad e_{2 n+1}\end{array}$ \\
\hline$C_{n}$ & $C_{n}$ & $\begin{array}{c}e_{j}-e_{j+1} \\
1 \leq j \leq n-1 \\
2 e_{n}\end{array}$ & 1 for all $\varepsilon$ & 2 & $2 e_{n}$ \\
\hline$D_{2 n}$ & $D_{2 n}$ & $\begin{array}{c}e_{j}-e_{j+1} \\
1 \leq j \leq 2 n-1 \\
e_{2 n-1}+e_{2 n}\end{array}$ & 1 for all $\varepsilon$ & 4 & $\begin{array}{l}e_{2 j-1}-e_{2 j} \\
1 \leq j \leq n \\
e_{2 n-1}+e_{2 n}\end{array}$ \\
\hline$D_{2 n+1}$ & $B_{2 n}$ & $\begin{array}{c}e_{j}-e_{j+1} \\
1 \leq j \leq 2 n-1 \\
e_{2 n}\end{array}$ & $\begin{array}{l}2 \text { for short } \varepsilon \\
1 \text { for long } \varepsilon\end{array}$ & 2 & $\begin{array}{l}e_{2 j-1}-e_{2 j} \\
1 \leq j \leq n\end{array}$ \\
\hline$E_{6}$ & $F_{4}$ & not needed & $\begin{array}{c}2 \text { for short } \varepsilon \\
1 \text { for long } \varepsilon\end{array}$ & 1 & $\varnothing$ \\
\hline$E_{7}$ & $E_{7}$ & $\begin{array}{l}\text { Roots } \varepsilon_{j} \text { with } \\
j \text { as in }\left(\begin{array}{c}2 \\
765431\end{array}\right)\end{array}$ & 1 for all $\varepsilon$ & 2 & $\varepsilon_{7}, \varepsilon_{5}, \varepsilon_{2}$ \\
\hline$E_{8}$ & $E_{8}$ & not needed & 1 for all $\varepsilon$ & 1 & $\varnothing$ \\
\hline$F_{4}$ & $F_{4}$ & not needed & 1 for all $\varepsilon$ & 1 & $\varnothing$ \\
\hline$G_{2}$ & $G_{2}$ & not needed & 1 for all $\varepsilon$ & 1 & $\varnothing$ \\
\hline
\end{tabular}

Proposition 5.4. Let $G$ be quasisplit with rank $G=\operatorname{rank} K$, and suppose that $G$ is acceptable in the sense of Harish-Chandra. Let $\sigma$ be defined as in Theorem 5.1, and let other notation be as above. Then all the members of $\mathcal{H}=\mathcal{H}_{\sigma}$ are simple roots.

Returning to $G$ not necessarily simple, let $\mathbf{d}_{\mathcal{H}}=\prod_{j=1}^{p} \mathbf{d}_{\xi_{j}}$ be the product of the Cayley transforms in all of the roots $\xi_{j} \in \mathcal{H}$. We define a new parabolic subgroup $Q=M A N$ by saying that $\mathbf{d}_{\mathcal{H}}$ leads from the data $\left(\mathfrak{m}_{\mathfrak{p}}, \mathfrak{a}_{\mathfrak{p}}, \mathfrak{b}_{\mathfrak{p}}\right)$ to data $(\mathfrak{m}, \mathfrak{a}, \mathfrak{b})$. Put $\widetilde{\xi}_{j}=\mathbf{d}_{\mathcal{H}}(\xi)$ for $1 \leq j \leq p$.

The only chamber in $i \mathfrak{b}_{\mathfrak{p}}$ is $i \mathfrak{b}_{\mathfrak{p}}$ itself. Because of the superorthogonality of the members of $\mathcal{H}$, it is apparent when $G$ is split that there are exactly $2^{p}$ chambers in $i \mathfrak{b}$. These are determined by specifying $p$ signs $s_{j}= \pm 1,1 \leq j \leq p$, and taking a chamber to be the set where the $p$ roots $s_{1} \widetilde{\xi}_{1}, \ldots, s_{p} \widetilde{\xi}_{p}$ are positive. When $G$ is merely quasisplit, the same conclusion is valid, but it is much less apparent. For 
the relevant fact we appeal to Lemma 8.5 of $\mathrm{KnZ}$, which shows that the only imaginary roots in $\Delta\left(\mathfrak{m}^{\mathbb{C}}, \mathfrak{b}^{\mathbb{C}}\right)$ are $\pm \widetilde{\xi}_{1}, \ldots, \pm \widetilde{\xi}_{p}$.

Let $W_{\mathcal{H}}$ be the group of order $2^{p}$ generated by the reflections in $\xi_{1}, \ldots, \xi_{p}$, and let

$$
E_{\mathcal{H}}=\left\{\begin{array}{l|l}
w \in W_{\mathcal{H}} & \begin{array}{l}
\text { for each } r \text { in } R_{\sigma}, w \text { and } r \text { have an even } \\
\text { number of factors } s_{\xi_{j}} \text { in common }
\end{array}
\end{array}\right\} .
$$

Using $\mathbf{d}_{\mathcal{H}}$, we can regard members of $W_{\mathcal{H}}$ as permuting the $2^{p}$ chambers of $i \mathfrak{b}$ simply transitively. The subgroup $E_{\mathcal{H}}$ gets identified with the set of members realizable in $M$. Theorems 8.7 and 12.3 of $\mathrm{KnZ}$ then yield the following.

Theorem 5.5. Let $G$ be quasisplit with rank $G=\operatorname{rank} K$, and suppose that $G$ is acceptable in the sense of Harish-Chandra. Let $\operatorname{ind}_{Q_{\mathfrak{p}}}^{G}(\sigma \otimes 1 \otimes 1)$ be defined as in Theorem 5.1, with character $\operatorname{ind}_{Q_{\mathfrak{p}}}^{G} \Theta^{M_{\mathfrak{p}} A_{\mathfrak{p}}}\left(0, i \mathfrak{b}_{\mathfrak{p}},\left.\sigma\right|_{F\left(B_{\mathfrak{p}}\right)}, 0\right)$, and let other notation be as above. Then (5.5) sets up a canonical isomorphism of $W_{\mathcal{H}} / E_{\mathcal{H}}$ onto the dual group $\widehat{R}_{\sigma}$. Moreover,

$$
\operatorname{ind}_{Q_{\mathfrak{p}}}^{G} \Theta^{M_{\mathfrak{p}} A_{\mathfrak{p}}}\left(0, i \mathfrak{b}_{\mathfrak{p}},\left.\sigma\right|_{F\left(B_{\mathfrak{p}}\right)}, 0\right)=\sum_{w \in W_{\mathcal{H}} / E_{\mathcal{H}} \cong \widehat{R}_{\sigma}} \operatorname{ind}_{Q}^{G} \Theta^{M A}\left(0, w C,\left.\sigma\right|_{F(B)}, 0\right)
$$

for any choice $C$ of the $2^{p}$ chambers in $i \mathfrak{b}$. The characters on the right side of (5.6) are all nonzero and irreducible, and they are given by nondegenerate data.

\section{Classification of totally Degenerate limits of Discrete Series}

In this section we give the classification of totally degenerate limits of discrete series, matching each such representation to one of the irreducible constituents of the principal series representation whose character appears on the left side of (5.6). Since the characters of the irreducible constituents have been written on the right side of (5.6) with nondegenerate data, this matching process will indeed complete the classification.

We continue with notation as in $\S 4$, and we shall also make use of the notation concerning the $R$ group introduced in $\S 5$. We use $\mathfrak{t}$ to denote a compact Cartan subalgebra of $\mathfrak{g}$ contained in $\mathfrak{k}$. A choice will be made in this section of a maximally noncompact $\theta$ stable Cartan subalgebra $\mathfrak{h}_{\mathfrak{p}}=\mathfrak{b}_{\mathfrak{p}} \oplus \mathfrak{a}_{\mathfrak{p}}$ of $\mathfrak{g}$, the choice depending on the totally degenerate limit of discrete series character that we are given. Then we let $M_{\mathfrak{p}}$ and $A_{\mathfrak{p}}$ be defined as usual. Once a lexicographic ordering has been introduced on $\mathfrak{a}_{\mathfrak{p}}^{\prime} \oplus i \mathfrak{b}_{\mathfrak{p}}^{\prime}$ such that every positive element of $\mathfrak{a}_{\mathfrak{p}}^{\prime}$ is greater than every element of $i \mathfrak{b}_{\mathfrak{p}}^{\prime}$, then we obtain a set $\Delta^{+}\left(\mathfrak{g}^{\mathbb{C}}, \mathfrak{h}_{\mathfrak{p}}^{\mathbb{C}}\right)$ of positive roots, a compatible set of positive restricted roots, and a subgroup $N_{\mathfrak{p}}$. We let $Q_{\mathfrak{p}}=M_{\mathfrak{p}} A_{\mathfrak{p}} N_{\mathfrak{p}}$.

The idea of the proof of the classification is to start with a totally degenerate limit of discrete series character $\Theta^{G}(0, C)$, construct a fundamental sequence $\widetilde{\alpha}_{1}, \ldots, \widetilde{\alpha}_{l}$ of strongly orthogonal noncompact roots relative to the positive system determined by $C$, and arrange that the generalized Schmid identities corresponding to the $l$ Cayley transforms $\mathbf{c}_{\widetilde{\alpha}_{j}}$ lead through the stage at the right side of (5.6) and onward to the principal series character on the left side of (5.6). Then in effect we can invert the last few generalized Schmid identities by means of the appropriate Cayley transforms $\mathbf{d}_{\alpha}$ and obtain the desired equality of irreducible characters. An example will illustrate what is supposed to happen. 
Example. In $D_{6}$, let the $C$-simple roots in $\Delta\left(\mathfrak{g}^{\mathbb{C}}, \mathfrak{t}^{\mathbb{C}}\right)$ be

$$
e_{1}-e_{2}, e_{2}-e_{3}, e_{3}-e_{4}, e_{4}-e_{5}, e_{5}-e_{6}, e_{5}+e_{6} \text {. }
$$

A fundamental sequence of strongly orthogonal noncompact roots is

$\widetilde{\alpha}_{1}=e_{1}-e_{2}, \widetilde{\alpha}_{2}=e_{3}-e_{4}, \widetilde{\alpha}_{3}=e_{5}-e_{6}, \widetilde{\alpha}_{4}=e_{1}+e_{2}, \widetilde{\alpha}_{5}=e_{3}+e_{4}, \widetilde{\alpha}_{6}=e_{5}+e_{6}$.

We apply each Cayley transform $\mathbf{c}_{\widetilde{\alpha}_{j}}$ and arrive at a maximally noncompact $\theta$ stable Cartan subalgebra $\mathfrak{h}_{\mathfrak{p}}=\mathfrak{b}_{\mathfrak{p}} \oplus \mathfrak{a}_{\mathfrak{p}}$. We put $\alpha_{j}=\mathbf{c}_{\widetilde{\alpha}_{j}}\left(\widetilde{\alpha}_{j}\right)$, but we leave the notation $e_{j}$ unchanged because there is little possibility of confusion. Our basis of $\mathfrak{a}_{\mathfrak{p}}^{\prime}$ is

$\alpha_{1}=e_{1}-e_{2}, \alpha_{2}=e_{3}-e_{4}, \alpha_{3}=e_{5}-e_{6}, \alpha_{4}=e_{1}+e_{2}, \alpha_{5}=e_{3}+e_{4}, \alpha_{6}=e_{5}+e_{6}$, and this is to be used lexicographically to define positive roots in $\Delta\left(\mathfrak{g}^{\mathbb{C}}, \mathfrak{h}_{\mathfrak{p}}^{\mathbb{C}}\right)$ and then to define $\sigma$ in Theorem 5.1. Computation shows that the resulting simple roots are

$$
e_{1}+e_{2},-e_{2}-e_{3}, e_{3}+e_{4},-e_{4}-e_{5}, e_{5}-e_{6}, e_{5}+e_{6} .
$$

On the other hand, Table 5.1 shows that $\mathcal{H}_{\sigma}$ consists of the first, third, fifth, and sixth simple roots in $\Delta^{+}\left(\mathfrak{g}^{\mathbb{C}}, \mathfrak{h}_{\mathfrak{p}}^{\mathbb{C}}\right)$. Thus

$$
\mathcal{H}_{\sigma}=\left\{e_{1}+e_{2}, e_{3}+e_{4}, e_{5}-e_{6}, e_{5}+e_{6}\right\}=\left\{\alpha_{3}, \alpha_{4}, \alpha_{5}, \alpha_{6}\right\} .
$$

We know from Theorems 5.1 and 5.2 that use of the six generalized Schmid identities corresponding to $\widetilde{\alpha}_{1}$ through $\widetilde{\alpha}_{6}$ leads us from our given representation to the principal series representation built from $\sigma$. Application of the inverted generalized Schmid identities corresponding to $\mathbf{d}_{\mathcal{H}_{\sigma}}$ takes us from the principal series representation to its irreducible constituents. Since $\mathcal{H}_{\sigma}$ consists of the last four of our $\alpha_{j}$ 's and since $\mathbf{d}_{\alpha_{j}}$ can be regarded as the inverse of $\mathbf{c}_{\tilde{\alpha}_{j}}$, it is reasonable to expect that we can get to the irreducible constituents of the principal series representation by using just the first two generalized Schmid identities, those corresponding to $\widetilde{\alpha}_{1}$ and $\widetilde{\alpha}_{2}$. Using just those two identities, we obtain an equality of our given totally degenerate limit of discrete series character with one of the characters on the right side of (5.6). Sorting matters out, we obtain the desired match.

There are two obstructions to having this process work in general. One is illustrated by changing the fundamental sequence in the above example to $\widetilde{\alpha}_{1}, \widetilde{\alpha}_{4}, \widetilde{\alpha}_{2}$, $\widetilde{\alpha}_{5}, \widetilde{\alpha}_{3}, \widetilde{\alpha}_{6}$. Computation shows that this change does not affect $\Delta^{+}\left(\mathfrak{g}^{\mathbb{C}}, \mathfrak{h}_{\mathfrak{p}}^{\mathbb{C}}\right)$. Thus we still have $\mathcal{H}_{\sigma}=\left\{e_{1}+e_{2}, e_{3}+e_{4}, e_{5}-e_{6}, e_{5}+e_{6}\right\}$, but the interpretation of $\mathcal{H}_{\sigma}$ in terms of the fundamental sequence is that $\mathcal{H}_{\sigma}$ consists of four of our $\alpha_{j}$ 's yet not the last four. This is not a serious problem, and Lemma 6.1 will address it satisfactorily.

The other obstruction is illustrated in $B_{3}$ by using the fundamental sequence

$$
\widetilde{\alpha}_{1}=e_{3}, \widetilde{\alpha}_{2}=e_{1}-e_{2}, \widetilde{\alpha}_{3}=e_{1}+e_{2} .
$$

We form $\alpha_{1}=e_{3}, \alpha_{2}=e_{1}-e_{2}, \alpha_{3}=e_{1}+e_{2}$ and find that the simple roots of $\Delta^{+}\left(\mathfrak{g}^{\mathbb{C}}, \mathfrak{h}_{\mathfrak{p}}^{\mathbb{C}}\right)$ are $e_{3}-e_{1}, e_{1}+e_{2},-e_{2}$. Table 5.1 says that $\mathcal{H}_{\sigma}$ consists of the first and third simple roots, thus of $e_{3}-e_{1}$ and $-e_{2}$. But neither of these roots is in our sequence. This is a more serious problem and will be addressed by Lemma 6.2 and the definition of "good fundamental sequence" that follows it.

In a reduced root system a simple root will be called isolated if it is orthogonal to all other simple roots in the system. Equivalently a simple root $\alpha$ in a system is isolated if there is no root $\beta$ in the system such that $\alpha+\beta$ is a root. Relative to a compact Cartan subalgebra and a positive system of roots for it, let $\widetilde{\alpha}_{1}, \ldots, \widetilde{\alpha}_{l}$ be 
a fundamental sequence. Let us say that $\widetilde{\alpha}_{j}$ is of the first kind if it is not isolated as a simple root in the system of roots orthogonal to $\widetilde{\alpha}_{1}, \ldots, \widetilde{\alpha}_{j-1}$, or of the second kind if it is isolated.

Lemma 6.1. Under the assumption that $\mathfrak{g}$ has a compact Cartan subalgebra $\mathfrak{t} \subseteq \mathfrak{k}$, let $\Delta^{+}\left(\mathfrak{g}^{\mathbb{C}}, \mathfrak{t}^{\mathbb{C}}\right)$ be a positive system for $\Delta\left(\mathfrak{g}^{\mathbb{C}}, \mathfrak{t}^{\mathbb{C}}\right)$, and suppose that

$$
\widetilde{\alpha}_{1}, \ldots, \widetilde{\alpha}_{l}
$$

is a fundamental sequence for $\Delta^{+}\left(\mathfrak{g}^{\mathbb{C}}, \mathfrak{t}^{\mathbb{C}}\right)$.

(a) If, for some $k$ with $1 \leq k \leq l-1, \widetilde{\alpha}_{k}$ is isolated as a simple root in the system of roots orthogonal to $\widetilde{\alpha}_{1}, \ldots, \widetilde{\alpha}_{k-1}$, then

$$
\widetilde{\alpha}_{1}, \ldots, \widetilde{\alpha}_{k-1}, \widetilde{\alpha}_{k+1}, \widetilde{\alpha}_{k}, \widetilde{\alpha}_{k+2}, \ldots, \widetilde{\alpha}_{l}
$$

is another fundamental sequence for $\Delta^{+}\left(\mathfrak{g}^{\mathbb{C}}, \mathfrak{t}^{\mathbb{C}}\right)$ and the type of each root-first kind or second kind - is not changed.

(b) Let $\mathbf{c}=\mathbf{c}_{\widetilde{\alpha}_{l}} \cdots \mathbf{c}_{\widetilde{\alpha_{1}}}$ be the composition of the commuting Cayley transforms from (6.1) or (6.2), and suppose that $\mathbf{c}$ leads from the data $(\mathfrak{g}, 0, \mathfrak{t})$ to $\left(\mathfrak{m}_{\mathfrak{p}}, \mathfrak{a}_{\mathfrak{p}}, \mathfrak{b}_{\mathfrak{p}}\right)$ and that $\alpha_{j}=\mathbf{c}\left(\widetilde{\alpha}_{j}\right)$ for $1 \leq j \leq l$ is the corresponding set of strongly orthogonal real roots in $\Delta\left(\mathfrak{g}^{\mathbb{C}}, \mathfrak{h}_{\mathfrak{p}}\right)$, where $\mathfrak{h}_{\mathfrak{p}}=\mathfrak{b}_{\mathfrak{p}} \oplus \mathfrak{a}_{\mathfrak{p}}$. Then the sequence of real roots $\alpha_{1}, \ldots, \alpha_{l}$ and the sequence obtained by applying $\mathbf{c}$ to (6.2) determine the same positive system $\Delta^{+}\left(\mathfrak{g}^{\mathbb{C}}, \mathfrak{h}_{\mathfrak{p}}^{\mathbb{C}}\right)$.

Proof. Define

$$
\begin{aligned}
\Delta_{k-1} & =\left\{\xi \in \Delta\left(\mathfrak{g}^{\mathbb{C}}, \mathfrak{t}^{\mathbb{C}}\right) \mid\left\langle\xi, \widetilde{\alpha}_{j}\right\rangle=0 \text { for } 1 \leq j \leq k-1\right\}, \\
\Delta_{k} & =\left\{\xi \in \Delta\left(\mathfrak{g}^{\mathbb{C}}, \mathfrak{t}^{\mathbb{C}}\right) \mid\left\langle\xi, \widetilde{\alpha}_{j}\right\rangle=0 \text { for } 1 \leq j \leq k\right\}, \\
\Delta_{k}^{\prime} & =\left\{\xi \in \Delta\left(\mathfrak{g}^{\mathbb{C}}, \mathfrak{t}^{\mathbb{C}}\right) \mid\left\langle\xi, \widetilde{\alpha}_{j}\right\rangle=0 \text { for } 1 \leq j \leq k-1 \text { and } j=k+1\right\} .
\end{aligned}
$$

The sequence (6.2) certainly consists of positive noncompact roots satisfying (i) and (iii) in the definition of fundamental sequence in $\S 3$. To show that (ii) holds for (6.2), we are to show that

(ii-a) $\widetilde{\alpha}_{k+1}$ is a simple root in $\Delta_{k-1}$, and

(ii-b) $\widetilde{\alpha}_{k}$ is a simple root in $\Delta_{k}^{\prime}$.

Condition (ii-b) is automatic since $\widetilde{\alpha}_{k}$ is simple in the larger system $\Delta_{k-1}$.

Arguing by contradiction, suppose that (ii-a) fails. Then $\widetilde{\alpha}_{k+1}=\beta+\beta^{\prime}$, where $\beta$ and $\beta^{\prime}$ are positive roots in $\Delta_{k-1}$. Let $\widetilde{\alpha}_{k}, \gamma_{1}, \ldots, \gamma_{m}$ be the simple roots in this subsystem. By assumption, $\widetilde{\alpha}_{k}$ is orthogonal to $\gamma_{1}, \ldots, \gamma_{m}$. We expand $\beta$ and $\beta^{\prime}$ in terms of $\widetilde{\alpha}_{k}, \gamma_{1}, \ldots, \gamma_{m}$ as $\beta=c_{0} \widetilde{\alpha}_{k}+\sum_{j=1}^{m} c_{j} \gamma_{j}$ and $\beta^{\prime}=c_{0}^{\prime} \widetilde{\alpha}_{k}+\sum_{j=1}^{m} c_{j}^{\prime} \gamma_{j}$ for some coefficients $\geq 0$, and we take the inner product of everything with $\widetilde{\alpha}_{k}$. Then we obtain $\left\langle\beta, \widetilde{\alpha}_{k}\right\rangle=c_{0}\left|\widetilde{\alpha}_{k}\right|^{2}$ and $\left\langle\beta^{\prime}, \widetilde{\alpha}_{k}\right\rangle=c_{0}^{\prime}\left|\widetilde{\alpha}_{k}\right|^{2}$ with $c_{0}$ and $c_{0}^{\prime}$ both $\geq 0$. Addition gives $0=\left\langle\widetilde{\alpha}_{k+1}, \widetilde{\alpha}_{k}\right\rangle=\left\langle\beta+\beta^{\prime}, \widetilde{\alpha}_{k}\right\rangle=\left(c_{0}+c_{0}^{\prime}\right)\left|\widetilde{\alpha}_{k}\right|^{2}$, and we conclude that $c_{0}=c_{0}^{\prime}=0$. Consequently $\beta$ and $\beta^{\prime}$ are linear combinations of $\gamma_{1}, \ldots, \gamma_{m}$, and so is $\widetilde{\alpha}_{k+1}$. Since $\gamma_{1}, \ldots, \gamma_{m}$ are by assumption orthogonal to $\widetilde{\alpha}_{k}$ and to $\widetilde{\alpha}_{1}, \ldots, \widetilde{\alpha}_{k-1}$, the root $\widetilde{\alpha}_{k+1}$ is exhibited as a nonsimple root in $\Delta_{k}$, and we have arrived at a contradiction. Thus (6.2) is a fundamental sequence.

To complete the proof of (a), we need to see that the type (first kind or second kind) of each of $\widetilde{\alpha}_{k}$ and $\widetilde{\alpha}_{k+1}$ is not affected by switching them in the sequence. We have seen that $\widetilde{\alpha}_{k}$ and $\widetilde{\alpha}_{k+1}$ are both simple in $\Delta_{k-1}$. We are given that $\widetilde{\alpha}_{k}$ is isolated in $\Delta_{k-1}$. Then no $\operatorname{root} \beta$ in $\Delta_{k-1}$ is such that $\beta+\widetilde{\alpha}_{k}$ is a root. In 
particular, $\beta+\widetilde{\alpha}_{k}$ cannot be a root if $\beta$ is in $\Delta_{k}^{\prime}$, and hence $\widetilde{\alpha}_{k}$ is isolated in $\Delta_{k}^{\prime}$. Thus $\widetilde{\alpha}_{k}$ is of the second kind in both sequences.

Similarly if $\widetilde{\alpha}_{k+1}$ is isolated in $\Delta_{k-1}$, then it is isolated in $\Delta_{k}$. Thus if $\widetilde{\alpha}_{k+1}$ is of the second kind in (6.2), it is of the second kind in (6.1). In the converse direction, suppose that $\widetilde{\alpha}_{k+1}$ is of the first kind in (6.2), i.e., that $\widetilde{\alpha}_{k+1}$ fails to be isolated in $\Delta_{k-1}$. Then $\beta+\widetilde{\alpha}_{k+1}$ is a root for some $\operatorname{root} \beta$ in $\Delta_{k-1}$. If $\left\langle\beta, \widetilde{\alpha}_{k}\right\rangle \neq 0$, then $\pm \beta+\widetilde{\alpha}_{k}$ is a root for some $\beta$ in $\Delta_{k-1}$ and some choice of sign, and this contradicts the assumption that $\widetilde{\alpha}_{k}$ is isolated in $\Delta_{k-1}$. So $\left\langle\beta, \widetilde{\alpha}_{k}\right\rangle=0$, and $\beta+\widetilde{\alpha}_{k+1}$ is a root with $\beta$ orthogonal to $\widetilde{\alpha}_{1}, \ldots, \widetilde{\alpha}_{k}$. Thus $\widetilde{\alpha}_{k+1}$ fails to be isolated in $\Delta_{k}$. This completes the proof of (a).

For (b) we argue by contradiction. If (b) is false, then there exists some root $\beta$ in $\Delta_{k-1}$ with $\left\langle\beta, \widetilde{\alpha}_{k}\right\rangle<0$ and $\left\langle\beta, \widetilde{\alpha}_{k+1}\right\rangle>0$. This root $\beta$ cannot be $-\widetilde{\alpha}_{k}$ since $\left\langle-\widetilde{\alpha}_{k}, \widetilde{\alpha}_{k+1}\right\rangle=0$, and thus $\beta+\widetilde{\alpha}_{k}$ is a root. Since $\beta$ is in $\Delta_{k}, \widetilde{\alpha}_{k}$ is not isolated in $\Delta_{k}$, in contradiction to the hypothesis. This proves (b).

Lemma 6.2. Under the assumptions that $\mathfrak{g}$ is quasisplit, is simple, has a compact Cartan subalgebra $\mathfrak{t} \subseteq \mathfrak{k}$, and has roots $\beta_{1}$ and $\beta_{2}$ with $\left|\beta_{1}\right|^{2}=2\left|\beta_{2}\right|^{2}$, suppose that $\Delta^{+}\left(\mathfrak{g}^{\mathbb{C}}, \mathfrak{t}^{\mathbb{C}}\right)$ is a positive system for $\Delta\left(\mathfrak{g}^{\mathbb{C}}, \mathfrak{t}^{\mathbb{C}}\right)$ in which every simple root is noncompact. Then $\mathfrak{g}$ has a fundamental sequence $\widetilde{\alpha}_{1}, \ldots, \widetilde{\alpha}_{l}$ of strongly orthogonal noncompact positive roots in which all of $\widetilde{\alpha}_{1}, \ldots, \widetilde{\alpha}_{l-1}$ are long. In any fundamental sequence, $\widetilde{\alpha}_{l}$ is of the second kind.

Remark. When $2 \leq m \leq n$, the conclusion of this lemma fails for $\mathfrak{g}=\mathfrak{s} \mathfrak{p}(m, n)$, which is not quasisplit.

Proof. The root system in question has to be of type $B_{n}, C_{n}$, or $F_{4}$. Since none of these systems has a nontrivial outer automorphism, $\mathfrak{g}$ has to be split. Thus the fundamental sequence has to be a basis for the roots. The only roots orthogonal to $\widetilde{\alpha}_{1}, \ldots, \widetilde{\alpha}_{l-1}$ are then the multiples of $\widetilde{\alpha}_{l}$; the root $\widetilde{\alpha}_{l}$ is simple in this system, and hence $\widetilde{\alpha}_{l}$ is of the second kind.

In split $C_{n}$, the fundamental sequence, in standard notation, has to consist of $2 e_{n}, 2 e_{n-1}, \ldots, 2 e_{1}$, and no short roots appear. In split $F_{4}$, every fundamental sequence consists of four long roots, and no short roots appear. In $B_{2 n}$, the sequence consisting of $e_{2 j-1}-e_{j}, e_{2 j-1}+e_{2 j}$ for $1 \leq j \leq n$ is fundamental with no short roots. Finally, in $B_{2 n+1}$, the sequence consisting of the roots for $B_{2 n}$ followed by $e_{2 n+1}$ is fundamental, and only the last member of the sequence is short.

If $\mathfrak{g}$ has a compact Cartan subalgebra $\mathfrak{t} \subseteq \mathfrak{k}$ and if a positive system $\Delta^{+}\left(\mathfrak{g}^{\mathbb{C}}, \mathfrak{t}^{\mathbb{C}}\right)$ is specified, a fundamental sequence $\widetilde{\alpha}_{1}, \ldots, \widetilde{\alpha}_{l}$ of strongly orthogonal positive noncompact roots will be said to be good if within each irreducible component of $\Delta\left(\mathfrak{g}^{\mathbb{C}}, \mathfrak{t}^{\mathbb{C}}\right)$ in which there are roots $\beta_{1}$ and $\beta_{2}$ with $\left|\beta_{1}\right|^{2}=2\left|\beta_{2}\right|^{2}$, at most one member of the part of the sequence within this component is short, and it is the last one for this component.

Lemma 6.3. Let $\mathfrak{g}$ be quasisplit and have a compact Cartan subalgebra $\mathfrak{t} \subseteq \mathfrak{k}$. Fix a positive system for $\Delta\left(\mathfrak{g}^{\mathbb{C}}, \mathfrak{t}^{\mathbb{C}}\right)$, and suppose that every simple root is noncompact. Then there exists a good fundamental sequence of positive noncompact roots.

Proof. Proposition 3.4 shows that a fundamental sequence exists. Fix an irreducible component $\Delta_{1}$ of $\Delta\left(\mathfrak{g}^{\mathbb{C}}, \mathfrak{t}^{\mathbb{C}}\right)$ in which there are roots $\beta_{1}$ and $\beta_{2}$ with $\left|\beta_{1}\right|^{2}=2\left|\beta_{2}\right|^{2}$. 
The subsequence of the fundamental sequence consisting just of those roots that lie in $\Delta_{1}$ is fundamental for that component, and Lemma 6.2 says that we can replace it by a good fundamental sequence for that component. Repeating this procedure for the other irreducible components of $\Delta\left(\mathfrak{g}^{\mathbb{C}}, \mathfrak{t}^{\mathbb{C}}\right)$, we obtain a good fundamental sequence for $\Delta\left(\mathfrak{g}^{\mathbb{C}}, \mathfrak{t}^{\mathbb{C}}\right)$.

Theorem 6.4. Let $G$ be quasisplit and acceptable in the sense of Harish-Chandra, and suppose that $\mathfrak{g}$ has a compact Cartan subalgebra $\mathfrak{t} \subseteq \mathfrak{k}$. Suppose that $\Theta^{G}(0, C)$ is a totally degenerate limit of discrete series character written in terms of $\mathfrak{t}$. Let $\widetilde{\alpha}_{1}, \ldots, \widetilde{\alpha}_{l}$ be a good fundamental sequence of strongly orthogonal positive noncompact roots relative to the positive system of $\Delta\left(\mathfrak{g}^{\mathbb{C}}, \mathfrak{t}^{\mathbb{C}}\right)$ determined by $C$. Let $\mathbf{c}=\mathbf{c}_{\widetilde{\alpha}_{1}} \cdots \mathbf{c}_{\widetilde{\alpha}_{l}}$ be the composition of Cayley transforms, and suppose that $\mathbf{c}$ leads from the data $(\mathfrak{g}, 0, \mathfrak{t})$ to data $\left(\mathfrak{m}_{\mathfrak{p}}, \mathfrak{a}_{\mathfrak{p}}, \mathfrak{b}_{\mathfrak{p}}\right)$ and that $\alpha_{j}=\mathbf{c}\left(\widetilde{\alpha}_{j}\right)$ for $1 \leq j \leq l$ is the resulting strongly orthogonal sequence of real roots in $\Delta\left(\mathfrak{g}^{\mathbb{C}}, \mathfrak{h}_{\mathfrak{p}}\right)$, where $\mathfrak{h}_{\mathfrak{p}}=\mathfrak{b}_{\mathfrak{p}} \oplus \mathfrak{a}_{\mathfrak{p}}$. Use the sequence $\alpha_{1}, \ldots, \alpha_{l}$ lexicographically to determine a unique positive system $\Delta^{+}\left(\mathfrak{g}^{\mathbb{C}}, \mathfrak{h}_{\mathfrak{p}}^{\mathbb{C}}\right)$, and define $\sigma$ to be the 1-dimensional representation of $M_{\mathfrak{p}}$ such that $\sigma$ is 1 on the identity component $\left(M_{\mathfrak{p}}\right)_{0}$ and $\sigma\left(\gamma_{\varepsilon}\right)$ equals -1 for every simple restricted root $\varepsilon$ with $m(\varepsilon)=1$. Let $\mathcal{H}_{\sigma}$ be the set of superorthogonal real roots defined in $\S 5$, and let $p=\left|\mathcal{H}_{\sigma}\right|$. Each member of $\mathcal{H}_{\sigma}$ is one of the roots $\alpha_{1}, \ldots, \alpha_{l}$, and the members of the sequence $\alpha_{1}, \ldots, \alpha_{l}$ can be permuted without changing $\Delta^{+}\left(\mathfrak{g}^{\mathbb{C}}, \mathfrak{h}_{\mathfrak{p}}^{\mathbb{C}}\right)$ so that the members of $\mathcal{H}_{\sigma}$ are the last $p$, namely $\alpha_{l-p+1}, \ldots, \alpha_{l}$. Define $\mathbf{d}_{\mathcal{H}}$ to be the composition $\prod_{\alpha \in \mathcal{H}_{\sigma}} \mathbf{d}_{\alpha}$, and suppose that $\mathbf{d}_{\mathcal{H}}$ leads from the data $\left(\mathfrak{m}_{\mathfrak{p}}, \mathfrak{a}_{\mathfrak{p}}, \mathfrak{b}_{\mathfrak{p}}\right)$ to data $(\mathfrak{m}, \mathfrak{a}, \mathfrak{b})$ as in $\S 5$. Then

$$
\Theta^{G}(0, C)=\operatorname{ind}_{Q}^{G} \Theta^{M A}\left(0, C_{M},\left.\sigma\right|_{F(B)}, 0\right),
$$

where $C_{M}$ is the unique chamber in $i \mathfrak{b}$ for which the $p$ roots $\mathbf{d}_{\mathcal{H}}(\alpha), \alpha \in \mathcal{H}_{\sigma}$, are $C_{M}$-positive, and (6.3) exhibits the given totally degenerate limit of discrete series as rewritten with nondegenerate data.

Remarks. The sequence $\widetilde{\alpha}_{1}, \ldots, \widetilde{\alpha}_{l}$ exists by Lemma 6.3. The transformed sequence $\alpha_{1}, \ldots, \alpha_{l}$ determines lexicographically a unique positive system $\Delta^{+}\left(\mathfrak{g}^{\mathbb{C}}, \mathfrak{h}_{\mathfrak{p}}^{\mathbb{C}}\right)$ by Proposition 3.2. The representation $\sigma$ exists and is unique according to Theorem 5.1. In the proof we may assume that the root system contains no component $G_{2}$ because Table 5.1 shows that the $R$ group is trivial for $G_{2}$.

Proof. We begin by adjusting the given sequence $\widetilde{\alpha}_{1}, \ldots, \widetilde{\alpha}_{l}$ in $\Delta\left(\mathfrak{g}^{\mathbb{C}}, \mathfrak{t}^{\mathbb{C}}\right)$ a little. Lemma 6.2 says that the exceptional short roots are of the second kind, and Lemma 6.1 allows us to move them to the end of the sequence without affecting $\Delta^{+}\left(\mathfrak{g}^{\mathbb{C}}, \mathfrak{h}_{\mathfrak{p}}^{\mathbb{C}}\right)$. A second application of Lemma 6.1 allows us to move all remaining members of the sequence that are of the second kind to the end of the sequence but just before the exceptional short roots. Roots of the first kind are still of the first kind, roots of the second kind are still of the second kind, and $\Delta^{+}\left(\mathfrak{g}^{\mathbb{C}}, \mathfrak{h}_{\mathfrak{p}}^{\mathbb{C}}\right)$ is unchanged. At the end of the adjustments, all the roots of the first kind precede all the roots of the second kind, and all the ordinary roots of the second kind precede all the exceptional short roots of the second kind. The sequence is still good. Let us retain the names $\widetilde{\alpha}_{1}, \ldots, \widetilde{\alpha}_{l}$ for the adjusted sequence, and let us refer to a member $\alpha_{j}$ of the corresponding sequence $\alpha_{1}, \ldots, \alpha_{l}$ as being first kind, second kind, or exceptional short if $\widetilde{\alpha}_{j}$ has that property. 
As in the proof of Proposition 4.3, let $\Sigma_{k}$ be the subsystem of restricted roots orthogonal to $\alpha_{1}, \ldots, \alpha_{k}$. We saw in that proof that the simple restricted roots needed for the expansion of any member of $\Sigma_{k}$ are in $\Sigma_{k}$. For each $k$, the restriction to $\mathfrak{a}_{\mathfrak{p}}^{\prime}$ of the real root $\alpha_{k}$ lies in $\Sigma_{k-1}$ but not in $\Sigma_{k}$. Thus $\Sigma_{k-1}$ contains at least one more simple restricted root than $\Sigma_{k}$ does. Since the total number of simple restricted roots coincides with the total number of $\alpha_{j}$ 's, it follows for each $j$ with $1 \leq j \leq l$ that there exists one and only one simple restricted root whose expansion in terms of $\alpha_{1}, \ldots, \alpha_{l}$ begins with a positive multiple of $\alpha_{j}$. Let us write $\varepsilon_{j}$ for this simple restricted root.

Let $\mathcal{H}_{\sigma}$ be the set of superorthogonal real roots defined in $\S 5$. Proposition 5.4 observes that each such root $\beta$ is simple in $\Delta^{+}\left(\mathfrak{g}^{\mathbb{C}}, \mathfrak{h}_{\mathfrak{p}}^{\mathbb{C}}\right)$. The main step in the proof is to show that $\beta$ is one of the roots $\alpha_{j}$ and is of the second kind.

If $\beta$ is in $\mathcal{H}_{\sigma}$, then $\beta$ is simple. Consequently, as we observed before Lemma 4.1, a theorem of Satake Sa] shows that the restriction of $\beta$ to $\mathfrak{a}_{\mathfrak{p}}^{\prime}$ is a simple restricted root. In the notation above, the restriction of $\beta$ is therefore of the form $\varepsilon_{j}$ for some unique $j$. Arguing by contradiction, suppose that some member of $\mathcal{H}_{\sigma}$ is not one of $\alpha_{1}, \ldots, \alpha_{l}$. Among all $\beta$ in $\mathcal{H}_{\sigma}$, we can then choose the one $\beta=\beta_{0}$ whose associated $\varepsilon_{j}$ has $j$ as small as possible so that $\varepsilon_{j} \neq \alpha_{j}$. Let $i$ be this smallest value of $j$. Fix an element $w$ of $R_{\sigma}$ having the reflection $s_{\beta_{0}}$ as one of its commuting factors.

We expand this real root $\beta_{0}$ in terms of $\alpha_{1}, \ldots, \alpha_{l}$ and form the irreducible root subsystem to which $\beta_{0}$ belongs. We saw that we may disregard split $G_{2}$. Applying Proposition 3.2, we obtain a list of possibilities for the expansion of $\beta_{0}$. Since $\beta_{0}$ begins with a positive multiple of $\alpha_{i}$, is not $\alpha_{i}$ itself, and is real, the only possibilities are (3.1) through (3.4). The coefficients of any of $\alpha_{r}, \alpha_{s}$, and $\alpha_{t}$ that appear have to be $<0$ since $\beta_{0}$ is simple. An expansion (3.3) cannot occur since the condition $\left|\alpha_{i}\right|<\left|\alpha_{r}\right|$ in (3.3) is incompatible with the fact that the fundamental sequence is good. Thus the only possibilities are (3.1), (3.2), and (3.4).

If $\beta_{0}$ is of the form (3.4), we can write $\beta_{0}=\frac{1}{2} \alpha_{i}-\frac{1}{2} \alpha_{r}$ with $i<r$ and with $\left|\beta_{0}\right|<\left|\alpha_{r}\right|=\left|\alpha_{i}\right|$. We have $s_{\beta_{0}}\left(\alpha_{i}\right)=\alpha_{r}$, and (4.2) gives $\gamma_{\beta_{0}} \gamma_{\alpha_{i}}=\gamma_{\alpha_{r}}$. The definition of $\sigma$ makes $\sigma\left(\gamma_{\beta_{0}}\right)=-1$, and thus exactly one of $\sigma\left(\gamma_{\alpha_{i}}\right)$ or $\sigma\left(\gamma_{\alpha_{r}}\right)$ is +1 . Since the element $w$ of $R_{\sigma}$ is a product of reflections in members of $\mathcal{H}_{\sigma}$, we can write $w=s_{\beta_{0}} s_{\beta_{1}} \cdots s_{\beta_{m}}$ with $\beta_{1}, \ldots, \beta_{m}$ in $\mathcal{H}_{\sigma}$ and with no repeated factors in the expansion. The associated simple $\varepsilon_{j}$ 's for $\beta_{l}$ with $l \geq 1$ have $j>i$ or else $\beta_{l}$ is some $\alpha_{k}$ with $k<i$, and each such $\beta_{l}$ is strongly orthogonal to $\beta_{0}$. Since each $\beta_{j}$ with $j \geq 1$ is orthogonal to $\beta_{0}$ and does not contain $\alpha_{i}$ in its expansion, no $\beta_{j}$ for $j \geq 1$ contains $\alpha_{r}$ in its expansion either. So we have $w\left(\alpha_{i}\right)=s_{\beta_{m}} \cdots s_{\beta_{1}} s_{\beta_{0}}\left(\alpha_{i}\right)=$ $s_{\beta_{m}} \cdots s_{\beta_{1}}\left(\alpha_{r}\right)=\alpha_{r}$ and similarly $w\left(\alpha_{r}\right)=\alpha_{i}$. If $\sigma\left(\gamma_{\alpha_{i}}\right)=+1$, then the equalities $\sigma\left(\gamma_{\alpha_{r}}\right)=-1$ and $w\left(\alpha_{i}\right)=\alpha_{r}$ show that $w$ does not preserve the set of positive restricted roots $\eta$ for which $m(\eta)=1$ and $\sigma\left(\gamma_{\eta}\right)=+1$; if $\sigma\left(\gamma_{\alpha_{r}}\right)=+1$, then the equalities $\sigma\left(\gamma_{\alpha_{i}}\right)=-1$ and $w\left(\alpha_{r}\right)=\alpha_{i}$ similarly show that $w$ does not preserve the set of positive restricted roots $\eta$ for which $m(\eta)=1$ and $\sigma\left(\gamma_{\eta}\right)=+1$. We have seen that one of these alternatives must hold, and thus (3.4) leads to a contradiction. So $\beta_{0}$ is of the form (3.1) or (3.2). In particular, $\left|\beta_{0}\right|=\left|\alpha_{i}\right|$.

In the remark with Proposition 4.3, we observed that $2 \rho_{\alpha_{i}}$ is a multiple of $\alpha_{i}$. Let us digress to compute the parity of this multiple. We have to take into account $\alpha_{i}$ itself and all the forms of roots indicated by (3.1) through (3.6). From $\beta$ as in (3.1), we get a contribution from each of the eight combinations of signs, and the sum is $4 \alpha_{i}$. Similarly, when all signs are taken into account, we get $2 \alpha_{i}$ from (3.2) 
and $2 \alpha_{i}$ from (3.3), and we get $2 \alpha_{i}$ from (3.6) if $\pm \omega$ are both used. Thus $2 \rho_{\alpha_{i}}$ is the sum of an odd multiple of $\alpha_{i}$ and whatever contribution we get from (3.4) and (3.5). If (3.5) makes any contribution, then we have seen in the proof of Proposition 4.3 that the corresponding irreducible component of $\Sigma$ is of type $(B C)_{n}$, and that component contributes nothing to the group $R_{\sigma}$, according to Table 5.1. Thus if (3.5) makes any contribution to $2 \rho_{\alpha_{i}}$, then $\beta_{0}$ could not have been in $\mathcal{H}_{\sigma}$, and we would not have been considering index $i$.

Continuing our digression, let us see that (3.4) cannot make any contribution to $2 \rho_{\alpha_{i}}$ under the assumption that $\beta_{0}$ is in $\mathcal{H}_{\sigma}$. Suppose on the contrary that there exists a real root of the form $\beta=\frac{1}{2} \alpha_{i}-\frac{1}{2} \alpha_{r}$ with $|\beta|<\left|\alpha_{i}\right|=\left|\alpha_{r}\right|$. The restrictions to $\mathfrak{a}_{\mathfrak{p}}$ of $\beta$ and $\alpha_{i}$ are nonorthogonal nonproportional restricted roots, and they are in the same irreducible component of $\Sigma$ as the restriction of $\beta_{0}$. This component must therefore be of one of the types $(B C)_{n}, F_{4}, C_{n}$, or $B_{n}$. We can rule out $(B C)_{n}$ and $F_{4}$ since Table 5.1 shows that such a component contributes nothing to $R_{\sigma}$ and leads to the conclusion that $\beta_{0}$ is not in $\mathcal{H}_{\sigma}$. Since $\alpha_{i}$ and $\beta_{0}$ are long, nonorthogonal, and nonproportional, we can rule out $C_{n}$. Thus the component of $\beta_{0}, \alpha_{i}$, and $\beta$ in $\Sigma$ may be assumed to be of type $B_{n}$. If $\sigma\left(\gamma_{\beta}\right)=-1$, then the same argument that rules out $\beta_{0}$ of the form (3.4) rules out the existence of $\beta$ now. Thus $\sigma\left(\gamma_{\beta}\right)=+1$. Since $\beta$ is short, it is conjugate via reflections in long simple restricted roots to plus or minus a short simple root. Taking (4.3) into account, we see that $\gamma_{\beta}$ equals $\gamma_{\varepsilon}$ for the unique short simple restricted root $\varepsilon$, and $\varepsilon$ has $m(\varepsilon)=1$. But then $+1=\sigma\left(\gamma_{\beta}\right)=\sigma\left(\gamma_{\varepsilon}\right)=-1$ by definition of $\sigma$, and we have arrived at a contradiction. Thus we may assume that no root of the form (3.4) occurs for our index $i$ if $\beta_{0}$ is as in (3.1) or (3.2) and $\beta_{0}$ belongs to $\mathcal{H}_{\sigma}$.

The upshot of our digression is that we may assume that

$$
(-1)^{2\left\langle\rho_{\alpha_{i}}, \alpha_{i}\right\rangle /\left|\alpha_{i}\right|^{2}}=-1
$$

Therefore Proposition 4.3 shows that

$$
\sigma\left(\gamma_{\alpha_{i}}\right)=-1
$$

Moreover, we already saw that $\beta_{0}$ has to be of the form (3.1) or (3.2).

Suppose that $\beta_{0}$ is of the form (3.1), namely $\beta_{0}=\frac{1}{2} \alpha_{i}-\frac{1}{2} \alpha_{r}-\frac{1}{2} \alpha_{s}-\frac{1}{2} \alpha_{t}$. Put $\beta_{0}^{\prime}=\frac{1}{2} \alpha_{i}+\frac{1}{2} \alpha_{r}+\frac{1}{2} \alpha_{s}+\frac{1}{2} \alpha_{t}$. From $\beta_{0}^{\prime}=-s_{\alpha_{i}}\left(\beta_{0}\right)$ and (4.3), we obtain

$$
\sigma\left(\gamma_{\beta_{0}^{\prime}}\right)=\sigma\left(\gamma_{\alpha_{i}}\right) \sigma\left(\gamma_{\beta_{0}}\right) .
$$

Because $\sigma\left(\gamma_{\beta_{0}}\right)=-1,(6.4)$ and (6.5) allow us to conclude that $\sigma\left(\gamma_{\beta_{0}^{\prime}}\right)=+1$. Since $s_{\beta_{0}}\left(\beta_{0}^{\prime}\right)=\alpha_{i}$ and since all roots other than $\beta_{0}$ that contribute to the element $w$ of $R_{\sigma}$ either begin their expansions after $\alpha_{i}$ or else are of the form $\alpha_{k}$ with $k<i$, we obtain $w\left(\beta_{0}^{\prime}\right)=\alpha_{i}$. This equality gives a contradiction because, by (6.4), it exhibits $w$ as not preserving the set of positive restricted roots $\varepsilon$ with $m(\varepsilon)=1$ and $\sigma\left(\gamma_{\varepsilon}\right)=+1$. Thus $\beta_{0}$ cannot be of the form (3.1).

Suppose that $\beta_{0}$ is of the form (3.2), namely $\beta_{0}=\frac{1}{2} \alpha_{i}-\frac{1}{2} \alpha_{r}-\frac{1}{2} \alpha_{s}$ with $\left|\beta_{0}\right|=$ $\left|\alpha_{i}\right|=\left|\alpha_{r}\right|>\left|\alpha_{s}\right|$. Put $\beta_{0}^{\prime}=\frac{1}{2} \alpha_{i}+\frac{1}{2} \alpha_{r}+\frac{1}{2} \alpha_{s}$. From $\beta_{0}^{\prime}=-s_{\alpha_{i}}\left(\beta_{0}\right)$, (4.3), and (6.4), we obtain

$$
\sigma\left(\gamma_{\beta_{0}^{\prime}}\right)=\sigma\left(\gamma_{\alpha_{i}}\right) \sigma\left(\gamma_{\beta_{0}}\right)=(-1)(-1)=+1
$$


From $s_{\beta_{0}}\left(\beta_{0}^{\prime}\right)=\alpha_{i}$, we deduce $w\left(\beta_{0}^{\prime}\right)=\alpha_{i}$, and we again obtain a contradiction. Consequently $\beta_{0}$ cannot be of the form (3.2), and all possibilities for $\beta_{0}$ are ruled out. We conclude that every member of $\mathcal{H}_{\sigma}$ is of the form $\alpha_{j}$ for some $j$.

Now let $\alpha_{j}$ be a member of $\mathcal{H}_{\sigma}$. We know that $\alpha_{j}$ is simple for $\Delta^{+}\left(\mathfrak{g}^{\mathbb{C}}, \mathfrak{h}_{\mathfrak{p}}^{\mathbb{C}}\right)$. Let us see that it is of the second kind, i.e., that it is isolated in the system of roots orthogonal to $\alpha_{1}, \ldots, \alpha_{j-1}$. This condition means that $\alpha_{j}$ is the only positive root for $\Delta^{+}\left(\mathfrak{g}^{\mathbb{C}}, \mathfrak{h}_{\mathfrak{p}}^{\mathbb{C}}\right)$ whose expansion in terms of $\alpha_{1}, \ldots, \alpha_{l}$ and $i \mathfrak{b}_{\mathfrak{p}}^{\prime}$ begins with a positive multiple of $\alpha_{j}$. If $\beta$ is a positive root whose expansion begins with $\frac{1}{2} \alpha_{j}$, then the same thing is true of $-s_{\alpha_{j}}(\beta)$, and $\alpha_{j}=\beta+\left(-s_{\alpha_{j}}(\beta)\right)$ gives the contradiction that $\alpha_{j}$ is not simple for $\Delta^{+}\left(\mathfrak{g}^{\mathbb{C}}, \mathfrak{h}_{\mathfrak{p}}^{\mathbb{C}}\right)$. If $\beta$ is a positive root whose expansion begins with $\alpha_{i}$, then $\beta=\alpha_{i}+\xi$, where $\xi$ is a root orthogonal to $\alpha_{i}$. If $\xi$ is negative, then $\alpha_{i}=\beta+(-\xi)$ exhibits $\alpha_{i}$ as not simple, contradiction. If $\xi$ is positive, then $-s_{\alpha_{i}}(\beta)$ is positive and $s_{\alpha_{i}}(\xi)=\xi$, so that $\alpha_{i}=-s_{\alpha_{i}}(\beta)+\xi$ exhibits $\alpha_{i}$ as not simple, contradiction. Consequently $\alpha_{j}$ is of the second kind.

Let $p$ be the number of roots in $\mathcal{H}_{\sigma}$. Because of the result of the previous paragraph, Lemma 6.1 allows us to adjust the fundamental sequence $\widetilde{\alpha}_{1}, \ldots, \widetilde{\alpha}_{l}$ further so that the $p$ roots $\widetilde{\alpha}_{j}$ with $\alpha_{j} \in \mathcal{H}_{\sigma}$ are the last $p$ members of the sequence. Lemma 6.1 says also that the positive system $\Delta^{+}\left(\mathfrak{g}^{\mathbb{C}}, \mathfrak{h}_{\mathfrak{p}}^{\mathbb{C}}\right)$ is unchanged.

Let us restate our construction in a way that takes into account all the different ways we were led to $\Delta^{+}\left(\mathfrak{g}^{\mathbb{C}}, \mathfrak{h}_{\mathfrak{p}}^{\mathbb{C}}\right)$. Starting from the chamber $C$ in $i \mathfrak{t}$, we formed a fundamental sequence $\widetilde{\alpha}_{1}, \ldots, \widetilde{\alpha}_{l}$ relative to $\Delta^{+}\left(\mathfrak{g}^{\mathbb{C}}, \mathfrak{t}^{\mathbb{C}}\right)$. For $\mathbf{c}$ equal to the composition of all the $\mathbf{c}_{\widetilde{\alpha}_{j}}$, we used $\mathbf{c}$ to pass from the data $(\mathfrak{g}, 0, \mathfrak{t})$ to data $\left(\mathfrak{m}_{\mathfrak{p}}, \mathfrak{a}_{\mathfrak{p}}, \mathfrak{b}_{\mathfrak{p}}\right)$ and to define $\alpha_{j}=\mathbf{c}\left(\widetilde{\alpha}_{j}\right)$ as a member of $\Delta\left(\mathfrak{g}^{\mathbb{C}}, \mathfrak{h}_{\mathfrak{p}}^{\mathbb{C}}\right)$. The sequence $\alpha_{1}, \ldots, \alpha_{l}$ enabled us to introduce a lexicographic ordering and define $\Delta^{+}\left(\mathfrak{g}^{\mathbb{C}}, \mathfrak{h}_{\mathfrak{p}}^{\mathbb{C}}\right)$. In terms of this positive system we could define $\sigma$. The group $R_{\sigma}$ relative to this same positive system led us to $\mathcal{H}_{\sigma}$, which consists of $\alpha_{l-p+1}, \ldots, \alpha_{l}$. From $\S 5$ we know that all the totally degenerate limits of discrete series lie in the induced representation from $\sigma$ and that the characters of the irreducible constituents of this induced representation are realized with nondegenerate data as $\operatorname{ind}_{Q}^{G} \Theta^{M A}\left(0, C_{M},\left.\sigma\right|_{F(B)}, 0\right)$, where the Cayley transform $\mathbf{d}_{\mathcal{H}}=\mathbf{d}_{\alpha_{l-p+1}} \cdots \mathbf{d}_{\alpha_{l}}$ leads from the data $\left(\mathfrak{m}_{\mathfrak{p}}, \mathfrak{a}_{\mathfrak{p}}, \mathfrak{b}_{\mathfrak{p}}\right)$ to the data $(\mathfrak{m}, \mathfrak{a}, \mathfrak{b})$. Since $\mathbf{c}_{\widetilde{\alpha}_{j}}$ and $\mathbf{d}_{\alpha_{j}}$ can be arranged to be exact inverses of one another, we can pass from the data $(\mathfrak{g}, 0, \mathfrak{t})$ to the data $(\mathfrak{m}, \mathfrak{a}, \mathfrak{b})$ more directly by the composition $\mathbf{c}_{\mathcal{H}}=\mathbf{c}_{l-p} \cdots \mathbf{c}_{1}$. The inverted generalized Schmid identities are exactly that - the same identities read from right to left instead of left to right. Consequently the generalized Schmid identities for the factors of $\mathbf{c}_{\mathcal{H}}$ show us how to pass from our given totally degenerate character $\Theta^{G}(0, C)$ to a situation where the character has been rewritten with nondegenerate data.

Now we can prove the conclusions of the theorem. What we have to do is identify the correct chamber $C_{M}$ of $i \mathfrak{b}$ in terms of the starting chamber $C$. Let us follow what happens to the chamber through each use of some $\mathbf{c}_{\widetilde{\alpha}_{j}}$. Say that the relevant Cartan subalgebra is $\mathfrak{h}_{j}=\mathfrak{b}_{j} \oplus \mathfrak{a}_{j}$ after using $\mathbf{c}_{\alpha_{j}} \cdots \mathbf{c}_{\alpha_{1}}, 1 \leq j \leq l-p$. The chamber after using $\mathbf{c}_{\widetilde{\alpha}_{1}}$ is $C^{s_{\widetilde{\alpha}_{1}}}$, the unique Weyl chamber of $i \mathfrak{b}_{1}$ containing $\operatorname{Proj}_{i \mathfrak{b}_{1}}(C)$. Thus we have

$$
C^{s \widetilde{\alpha}_{1}} \supseteq \operatorname{Proj}_{i \mathfrak{b}_{1}}(C)
$$

Hence

$$
\left(C^{s \widetilde{\alpha}_{1}}\right)^{s_{\widetilde{\alpha}_{2}}} \supseteq \operatorname{Proj}_{i_{\mathfrak{b}_{2}}}\left(C^{s_{\widetilde{\alpha}_{1}}} \supseteq \operatorname{Proj}_{i \mathfrak{b}_{2}}\left(\operatorname{Proj}_{i \mathfrak{b}_{1}}(C)\right)=\operatorname{Proj}_{i \mathfrak{b}_{2}}(C)\right.
$$


Iterating, we obtain

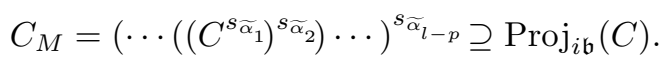

If $H$ is in $C$, then

$$
\operatorname{Proj}_{i \mathfrak{b}}(H)=H-\sum_{j=1}^{l-p} \frac{\widetilde{\alpha}_{j}(H)}{\left|\widetilde{\alpha}_{j}\right|^{2}} H_{\widetilde{\alpha}_{j}} .
$$

An imaginary positive root $\widetilde{\beta}$ of $\left(\mathfrak{m}^{\mathbb{C}}, \mathfrak{b}^{\mathbb{C}}\right)$ extends uniquely to an imaginary positive root of $\left(\mathfrak{g}^{\mathbb{C}}, \mathfrak{t}^{\mathbb{C}}\right)$ that is orthogonal to $\widetilde{\alpha}_{1}, \ldots, \widetilde{\alpha}_{l-p}$. Then $\widetilde{\beta}$ takes the same value on $H$ in $C$ that it takes on the two sides of (6.7). On $C$, it is positive, and we conclude from (6.6) that every such $\widetilde{\beta}$ is positive on a certain open subset of

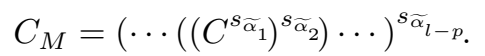

In particular, $\widetilde{\alpha}_{l-p+1}, \ldots, \widetilde{\alpha}_{l}$ are positive on this open set. We saw in Theorem 5.5 that the chambers of $i \mathfrak{b}$ are $2^{p}$ in number and are distinguished by the signs of $\widetilde{\alpha}_{l-p+1}, \ldots, \widetilde{\alpha}_{l}$ on them. The conclusion is that the desired chamber $C_{M}$ is the unique one where $\widetilde{\alpha}_{l-p+1}, \ldots, \widetilde{\alpha}_{l}$ are all positive, and the proof is complete.

\section{Particular cases}

1) Groups of type $A$. The groups in question of type $A$ are $G=S U(n, n)$ and $G=S U(n+1, n)$, and most of the development in $\S \S 4-6$ of this paper is unnecessary for these cases. For $S U(n+1, n)$, we see from Appendix C of Kn3. that $M_{\mathfrak{p}}$ is connected, and it follows that $\sigma$ is trivial; the unitary spherical principal series is irreducible for any $G$, and thus the only totally degenerate limit of discrete series representation is the spherical principal series representation with trivial $A_{\mathfrak{p}}$ parameter. The case of $S U(n, n)$ is only slightly more subtle. The group $M_{\mathfrak{p}}$ has two components in this case. A good fundamental sequence is $e_{1}-e_{2}, e_{3}-e_{4}, \ldots$, and we can check directly that all the intermediate $M$ groups are connected. If we think of applying generalized Schmid identities in succession corresponding to the Cayley transforms $\mathbf{c}_{\tilde{\alpha}}$, we see that the question comes down to what happens at the last step, specifically whether the applicable generalized Schmid identity is of type (a) or type (b). This is the question whether the last $s_{\widetilde{\alpha}}$ is in $W\left(B^{*}: M^{*}\right)$ or not. Direct computation shows that it is not, and the identity to use is of type (a). Then we get reducibility into two pieces, and this reducibility is understood at the last step. Thus we get nondegenerate data one step removed from the minimal parabolic subalgebra.

2) Other classical groups. The full theory is needed in most of the other classical cases, and the results are summarized in Table 7.1; the table also includes the exceptional cases and refers to them by Cartan's numbering, which is reproduced in Appendix $\mathrm{C}$ of $\mathrm{Kn} 3$. Let us think in terms of starting from a compact Cartan subalgebra and applying a succession of generalized Schmid identities. A curious feature of the classical cases is that the stage at which we find nondegenerate data, namely $\left|\mathcal{H}_{\sigma}\right|$ steps away from a minimal parabolic subalgebra, is always the first stage at which $\mathfrak{m}^{*}$ becomes the sum of an abelian subalgebra and a direct sum of copies of $\mathfrak{s l}(2, \mathbb{R})$. As the table shows, this coincidence is no longer valid for the exceptional groups, i.e., the entries in the last two columns of Table 7.1 are not equal for the exceptional groups. 
Table 7.1. Comparison of $\left|\mathcal{H}_{\sigma}\right|$ with the number of roots $\widetilde{\alpha}_{j}$ of the second kind in a standard good fundamental sequence

\begin{tabular}{|c|c|c|c|c|c|}
\hline$\Delta$ & $\mathfrak{g}$ & $\begin{array}{c}\text { Real } \\
\text { Rank }\end{array}$ & $\begin{array}{c}\text { Number } \widetilde{\alpha}_{j} \\
\text { Of } \\
\text { First Kind }\end{array}$ & $\begin{array}{c}\text { Number } \widetilde{\alpha}_{j} \\
\text { Of } \\
\text { Second Kind }\end{array}$ & $\left|\mathcal{H}_{\sigma}\right|$ \\
\hline$A_{2 n-1}$ & $\mathfrak{s u}(n, n)$ & $n$ & $n-1$ & 1 & 1 \\
$A_{2 n}$ & $\mathfrak{s u}(n+1, n)$ & $n$ & $n$ & 0 & 0 \\
$B_{2 n}$ & $\mathfrak{s o}(2 n+1,2 n)$ & $2 n$ & $n$ & $n$ & $n$ \\
$B_{2 n+1}$ & $\mathfrak{s o}(2 n+2,2 n+1)$ & $2 n+1$ & $n$ & $n+1$ & $n+1$ \\
$C_{n}$ & $\mathfrak{s p}(n, \mathbb{R})$ & $n$ & $n-1$ & 1 & 1 \\
$D_{2 n}$ & $\mathfrak{s o}(2 n, 2 n)$ & $2 n$ & $n-1$ & $n+1$ & $n+1$ \\
$D_{2 n+1}$ & $\mathfrak{s o}(2 n+2,2 n)$ & $2 n$ & $n$ & $n$ & $n$ \\
$E_{6}$ & $\mathrm{E} \mathrm{II}$ & 4 & 3 & 1 & 0 \\
$E_{7}$ & $\mathrm{E} \mathrm{V}$ & 7 & 3 & 4 & 3 \\
$E_{8}$ & $\mathrm{E} \mathrm{VIII}$ & 8 & 4 & 4 & 0 \\
$F_{4}$ & $\mathrm{~F} \mathrm{I}$ & 4 & 2 & 2 & 0 \\
$G_{2}$ & $\mathrm{G}$ & 2 & 1 & 1 & 0 \\
\hline
\end{tabular}

The computations in the classical cases are straightforward, particularly in the presence of Table 5.1. The case of $C_{n}$ is a little special in that the good fundamental sequence has to be $2 e_{n}, 2 e_{n-1}, \ldots, 2 e_{1}$. For the other classical cases, a good fundamental sequence is obtained by using as many as possible of the roots $e_{1}-e_{2}$, $e_{3}-e_{4}, \ldots$ and following them with the corresponding $e_{1}+e_{2}, e_{3}+e_{4}, \ldots$ In the case of $B_{2 n+1}$, one adjoins $e_{2 n+1}$ at the end.

3) Split $E_{7}$. The only exceptional simple group under study for which the $R$ group of the special $\sigma$ is nontrivial is split $E_{7}$, and some comments are in order about this case. A good fundamental sequence is constructed as follows. Fix $C$. Number the $C$-simple roots in the Dynkin diagram as $\beta_{j}$ with $j$ as in $\left(\begin{array}{c}2 \\ 765431\end{array}\right)$. Put

$$
\begin{aligned}
& \widetilde{\alpha}_{1}=\beta_{7}, \\
& \widetilde{\alpha}_{2}=\widetilde{\alpha}_{1}+2 \beta_{6}+2 \beta_{5}+2 \beta_{4}+\beta_{3}+\beta_{2}, \\
& \widetilde{\alpha}_{3}=\beta_{3}, \\
& \widetilde{\alpha}_{4}=\widetilde{\alpha}_{2}+\beta_{5}+2 \beta_{4}+2 \beta_{3}+\beta_{2}+2 \beta_{1}, \\
& \widetilde{\alpha}_{5}=\beta_{5}+2 \beta_{4}+\beta_{3}+\beta_{2}, \\
& \widetilde{\alpha}_{6}=\beta_{5}, \\
& \widetilde{\alpha}_{7}=\beta_{2} .
\end{aligned}
$$

During the construction, the system orthogonal to $\widetilde{\alpha}_{1}$ is of type $D_{6}$, with simple roots consisting of $\beta_{1}, \beta_{2}, \beta_{3}, \beta_{4}, \beta_{5}$, and $\widetilde{\alpha}_{2}$. The subsystem orthogonal to $\widetilde{\alpha}_{2}$ is of type $D_{4}+A_{1}$, with the simple roots of $D_{4}$ consisting of $\beta_{2}, \beta_{3}, \beta_{4}, \beta_{5}$ and with $A_{1}$ formed from $\widetilde{\alpha}_{5}$. The subsystem orthogonal to $\widetilde{\alpha}_{3}=\beta_{3}$ has four isolated simple roots, namely $\widetilde{\alpha}_{4}, \widetilde{\alpha}_{5}, \beta_{2}$, and $\beta_{5}$. These remarks explain the entries in the fourth and fifth columns of Table 7.1 for $E_{7}$.

To trace what happens for $E_{7}$ in Theorem 6.4, we remove the tildes from the roots $\widetilde{\alpha}_{j}$ and use the roots $\alpha_{j}$ to impose a lexicographic ordering and obtain $\Delta^{+}\left(\mathfrak{g}^{\mathbb{C}}, \mathfrak{h}_{\mathfrak{p}}^{\mathbb{C}}\right)$. Examining the proof of Proposition 4.3, we can quickly write the expansions of the 
simple roots $\xi_{j}$ in this system in terms of the basis $\alpha_{1}, \ldots, \alpha_{8}$ as

$$
\begin{aligned}
& \xi_{1}=\frac{1}{2}\left(\alpha_{1}-\alpha_{2}-\alpha_{3}-\alpha_{4}\right), \\
& \xi_{2}=\frac{1}{2}\left(\alpha_{2}-\alpha_{3}-\alpha_{5}-\alpha_{6}\right), \\
& \xi_{3}=\frac{1}{2}\left(\alpha_{3}-\alpha_{4}-\alpha_{5}-\alpha_{7}\right), \\
& \xi_{4}=\alpha_{4}, \\
& \xi_{5}=\alpha_{5}, \\
& \xi_{6}=\alpha_{6}, \\
& \xi_{7}=\alpha_{7} .
\end{aligned}
$$

Computing the inner products $\left\langle\xi_{i}, \xi_{j}\right\rangle$, we can form a Dynkin diagram. Then we see that the numbering of the simple roots as $\xi_{j}$ corresponds to $j$ as in $\left(\begin{array}{c}7 \\ 625341\end{array}\right)$. Table 5.1 says that the roots in $\mathcal{H}_{\sigma}$ are the simple roots in the positions $\mathrm{X}$ in ( $\underset{\text { xox000 }}{\mathrm{X}}$ ). These are the roots $\xi_{5}=\alpha_{5}, \xi_{6}=\alpha_{6}$, and $\xi_{7}=\alpha_{7}$, and we have a confirmation of the prediction from the proof of Theorem 6.4 that the members of $\mathcal{H}_{\sigma}$ all lie in the sequence $\alpha_{1}, \ldots, \alpha_{8}$ and are of the second kind.

\section{REFERENCES}

[Ca] H. Carayol, Limites dégénérées de séries discrètes, formes automorphes et variétés de Griffiths-Schmid: le cas du groupe U(2,1), Compositio Math., 111, 1998, pp. 51-88. MR1611063 (99k:22027)

[Cl] L. Clozel, On the cohomology of Kottwitz's arithmetic varieties, Duke J. Math., 72, 1993, pp. 757-795. MR1253624 (95b:11055)

[HC1] Harish-Chandra, Representations of a semisimple Lie group on a Banach space I, Trans. Amer. Math. Soc., 75, 1953, pp. 185-243. MR0056610 (15:100f)

[HC2] Harish-Chandra, Representations of semisimple Lie groups II, Trans. Amer. Math. Soc., 76, 1954, pp. 26-65. MR0058604 (15:398a)

[HC3] Harish-Chandra, Discrete series for semisimple Lie groups I, Acta Math., 113, 1965, pp. 241-318; Two theorems on semi-simple Lie groups, Ann. of Math., 83, 1956, pp. 74128; Discrete series for semisimple Lie groups II, Acta Math., 116, 1966, pp. 1-111. MR0219665 (36:2744) MR0194556 (33:2766) MR0219666 (36:2745)

[HC4] Harish-Chandra, On the theory of the Eisenstein integral, Conference on Harmonic Analysis, Springer Lecture Notes in Mathematics, 266, 1972, pp. 123-149. MR0399355(53:3200)

$[\mathrm{HeS}]$ H. Hecht and W. Schmid, A proof of Blattner's conjecture, Invent. Math., 31, 1975, pp. 129-154. MR0396855 (53:715)

[JoW] K. D. Johnson and N. R. Wallach, Composition series and intertwining operators for the spherical principal series I, Trans. Amer. Math. Soc., 229, 1977, pp. 137-173. MR0447483 $(56: 5794)$

[Kn1] A. W. Knapp, Weyl group of a cuspidal parabolic, Annales Sci. École Norm. Sup., 8, 1975, pp. 275-294. MR0376963 (51:13138)

[Kn2] A. W. Knapp, Commutativity of intertwining operators for semisimple groups, Compositio Math., 46, 1982, pp. 33-84. MR660154 (83i:22022)

[Kn3] A. W. Knapp, Lie Groups Beyond an Introduction, Birkhäuser, Boston, 1996, second edition, 2002. MR1399083 (98b:22002), MR1920389 (2003c:22001)

[KnW] A. W. Knapp and N. R. Wallach, Szegö kernels associated with discrete series, Invent. Math., 34, 1976, pp. 163-200; correction and addition, 62, 1980, pp. 341-346. MR0419686 (54:7704) MR0595593 (82i:22016)

[KnZ] A. W. Knapp and G. J. Zuckerman, Classification of irreducible tempered representations of semisimple groups, Annals of Math., 116, 1982, pp. 389-501; typesetter's correction, 119, 1984, p. 639. MR0672840 (84h:22034a) MR:0678478 (84h:22034b), MR0744867 (85e:22023) 
[La] R. P. Langlands, On the classification of irreducible representations of real algebraic groups, mimeographed notes, Institute for Advanced Study, 1973; in P. J. Sally and D. A. Vogan (eds.), Representation Theory and Harmonic Analysis on Semisimple Lie Groups, Math. Surveys and Monographs, 31, American Mathematical Society, Providence, 1989, pp. 101-170, MR1011897(91e:22017)

[Le] J. Lepowsky, Algebraic results on representations of semisimple Lie groups, Trans. Amer. Math. Soc., 176, 1973, pp. 1-44. MR0346093 (49:10819)

[Sa] I. Satake, On representations and compactifications of symmetric Riemannian spaces, Annals of Math., 71, 1960, pp. 77-110. MR0118775 (22:9546)

[So] W. Soergel, On the $\mathfrak{n}$-cohomology of limits of discrete series representations, Represent. Theory, 1, 1997, pp. 69-82. MR.1445512 (98h:22018)

[Tr] P. C. Trombi, The tempered spectrum of a real semisimple Lie group, Amer. J. Math., 99, 1977, pp. 57-75. MR0453929 (56:12182)

[Wa1] N. R. Wallach, Cyclic vectors and irreducibility for principal series representations, Trans. Amer. Math. Soc., 158, 1971, pp. 107-113. MR0281844 (43:7558)

[Wa2] N. R. Wallach, On the Selberg trace formula in the case of compact quotient, Bull. Amer. Math. Soc., 82, 1976, pp. 171-195. MR0404533 (53:8333)

[Zu] G. Zuckerman, Tensor products of finite and infinite dimensional representations of semisimple Lie groups, Ann. of Math., 106, 1977, pp. 295-308. MR0457636 (56:15841)

Université Louis Pasteur et C.N.R.S., I.R.M.A., 7 Rue René Descartes, 67084 StrasBourg Cedex, France

E-mail address: carayol@math.u-strasbg.fr

Department of Mathematics, State University of New York, Stony Brook, New York 11794

Current address: 81 Upper Sheep Pasture Road, East Setauket, New York 11733-1729

E-mail address: aknapp@math.sunysb.edu 